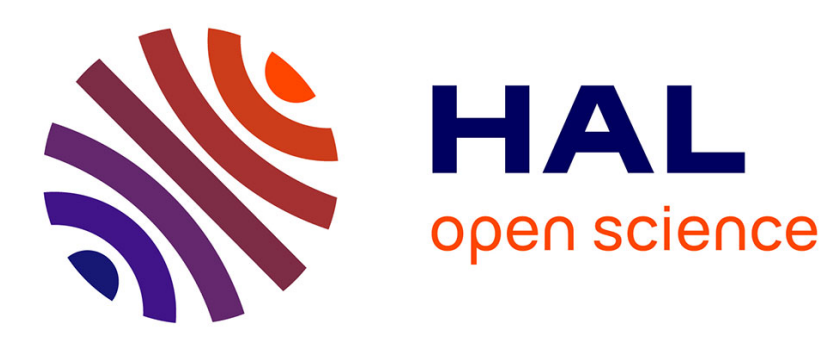

\title{
Étude observationnelle de facteurs prédictifs d'erreurs médicamenteuses et de critères de priorisation en conciliation médicamenteuse gériatrique
}

Mélissa Abbes

\section{- To cite this version:}

Mélissa Abbes. Étude observationnelle de facteurs prédictifs d'erreurs médicamenteuses et de critères de priorisation en conciliation médicamenteuse gériatrique. Sciences pharmaceutiques. 2021. dumas03211734

\section{HAL Id: dumas-03211734 https://dumas.ccsd.cnrs.fr/dumas-03211734}

Submitted on 29 Apr 2021

HAL is a multi-disciplinary open access archive for the deposit and dissemination of scientific research documents, whether they are published or not. The documents may come from teaching and research institutions in France or abroad, or from public or private research centers.
L'archive ouverte pluridisciplinaire HAL, est destinée au dépôt et à la diffusion de documents scientifiques de niveau recherche, publiés ou non, émanant des établissements d'enseignement et de recherche français ou étrangers, des laboratoires publics ou privés. 
Aix $*$ Marseille Université

\title{
THÈSE
}

PRESENTEE ET PUBLIQUEMENT SOUTENUE

DEVANT LA FACULTE DE PHARMACIE DE MARSEILLE

LE 20 AVRIL 2021

PAR

$M^{\text {elle }}$ ABBES Melissa

Née le 26/09/1991 à Nice

EN VUE D'OBTENIR

LE DIPLOME D'ETAT DE DOCTEUR EN PHARMACIE

ETUDE OBSERVATIONNELLE DE FACTEURS PREDICTIFS

D'ERREURS MEDICAMENTEUSES ET DE CRITERES

DE PRIORISATION EN CONCILIATION

MEDICAMENTEUSE GERIATRIQUE

\section{THÈSE ARTICLE}

\author{
JURY : \\ Président : $\quad$ M. le Professeur Pascal RATHELOT \\ Membres : $\quad$ Mme le Docteur Charlotte PAPAILHAU \\ Mme le Docteur Dorothée NAUDET \\ M. le Docteur Teddy NOVAIS
}



27 Boulevard Jean Moulin - 13385 MARSEILLE Cedex 05

Tel. : 0491835500 - Fax : 0491802612

\section{ADMINISTRATION :}

Doyen :

Vice-Doyens :

Chargés de Mission :

Conseiller du Doyen :

Doyens honoraires :

Professeurs émérites :

Professeurs honoraires :

Chef des Services Administratifs : Mme Florence GAUREL

Chef de Cabinet :

Mme Aurélie BELENGUER

Responsable de la Scolarité :

Mme Nathalie BESNARD
Mme Françoise DIGNAT-GEORGE

M. Jean-Paul BORG, M. François DEVRED, M. Pascal RATHELOT

Mme Pascale BARBIER, M. David BERGE-LEFRANC, Mme Manon CARRE, Mme Caroline DUCROS, Mme Frédérique GRIMALDI, M. Guillaume HACHE

M. Patrice VANELLE

M. Patrice VANELLE, M. Pierre TIMON-DAVID,

M. José SAMPOL, M. Athanassios ILIADIS, M. Henri PORTUGAL, M. Philippe CHARPIOT

M. Guy BALANSARD, M. Yves BARRA, Mme Claudette BRIAND, M. Jacques CATALIN, Mme Andrée CREMIEUX, M. Aimé CREVAT, M. Gérard DUMENIL, M. Alain DURAND, Mme Danielle GARÇON, M. Maurice JALFRE, M. Joseph JOACHIM, M. Maurice LANZA, M. José MALDONADO, M. Patrick REGLI, M. Jean-Claude SARI

\section{DEPARTEMENT BIO-INGENIERIE PHARMACEUTIQUE}

Responsable : Professeur Philippe PICCERELLE

\section{PROFESSEURS}

BIOPHYSIQUE

GENIE GENETIQUE ET BIOINGENIERIE

PHARMACIE GALENIQUE, PHARMACOTECHNIE INDUSTRIELLE, BIOPHARMACIE ET COSMETOLOGIE
M. Vincent PEYROT

M. Hervé KOVACIC

M. Christophe DUBOIS

M. Philippe PICCERELLE 
BIOPHYSIQUE

GENIE GENETIQUE ET BIOTECHNOLOGIE

PHARMACIE GALENIQUE, PHARMACOTECHNIE INDUSTRIELLE, BIOPHARMACIE ET COSMETOLOGIE

BIO-INGENIERIE PHARMACEUTIQUE ET BIOTHERAPIES PHARMACO ECONOMIE, E-SANTE
M. Robert GILLI

Mme Odile RIMET-GASPARINI

Mme Pascale BARBIER

M. François DEVRED

Mme Manon CARRE

M. Gilles BREUZARD

Mme Alessandra PAGANO

M. Eric SEREE-PACHA

Mme Véronique REY-BOURGAREL

M. Pascal PRINDERRE

M. Emmanuel CAUTURE

Mme Véronique ANDRIEU

Mme Marie-Pierre SAVELLI

M. Jérémy MAGALON

Mme Carole SIANI

\section{ENSEIGNANTS CONTRACTUELS}

ANGLAIS

Mme Angélique GOODWIN

DEPARTEMENT BIOLOGIE PHARMACEUTIQUE

Responsable : Professeur Françoise DIGNAT-GEORGE

\section{PROFESSEURS}

BIOLOGIE CELLULAIRE

HEMATOLOGIE ET IMMUNOLOGIE

MICROBIOLOGIE

PARASITOLOGIE ET MYCOLOGIE MEDICALE, HYGIENE ET ZOOLOGIE
M. Jean-Paul BORG

Mme Françoise DIGNAT-GEORGE

Mme Laurence CAMOIN-JAU

Mme Florence SABATIER-MALATERRE

Mme Nathalie BARDIN

M. Jean-Marc ROLAIN

M. Philippe COLSON

Mme Nadine AZAS-KREDER 
BIOCHIMIE FONDAMENTALE, MOLECULAIRE ET CLINIQUE

HEMATOLOGIE ET IMMUNOLOGIE

MICROBIOLOGIE

PARASITOLOGIE ET MYCOLOGIE MEDICALE, HYGIENE ET ZOOLOGIE

BIOLOGIE CELLULAIRE

ATER

BIOCHIMIE FONDAMENTALE, MOLECULAIRE ET CLINIQUE

BIOLOGIE CELLULAIRE ET MOLECULAIRE

HEMATOLOGIE ET IMMUNOLOGIE

Mme Mélanie VELIER

Mme Anne-Claire DUCHEZ

Mme Alexandra WALTON

A.H.U.
M. Thierry AUGIER

M. Edouard LAMY

Mme Alexandrine BERTAUD

Mme Claire CERINI

Mme Edwige TELLIER

M. Stéphane POITEVIN

Mme Aurélie LEROYER

$M$. Romaric LACROIX

Mme Sylvie COINTE

Mme Michèle LAGET

Mme Anne DAVIN-REGLI

Mme Véronique ROUX

$M$. Fadi BITTAR

Mme Isabelle PAGNIER

Mme Sophie EDOUARD

M. Seydina Mouhamadou DIENE

Mme Carole DI GIORGIO

$M$. Aurélien DUMETRE

Mme Magali CASANOVA

Mme Anita COHEN

Mme Anne-Catherine LOUHMEAU

DEPARTEMENT CHIMIE PHARMACEUTIQUE

Responsable: Professeur Patrice VANELLE

\section{PROFESSEURS}

CHIMIE ANALYTIQUE, QUALITOLOGIE ET NUTRITION

CHIMIE PHYSIQUE - PREVENTION DES RISQUES ET NUISANCES TECHNOLOGIQUES

CHIMIE MINERALE ET STRUCTURALE -

CHIMIE THERAPEUTIQUE

CHIMIE ORGANIQUE PHARMACEUTIQUE

PHARMACOGNOSIE, ETHNOPHARMACOGNOSIE
Mme Catherine BADENS

M. David BERGE-LEFRANC

M. Pascal RATHELOT

M. Maxime CROZET

M. Patrice VANELLE

M. Thierry TERME

Mme Evelyne OLLIVIER 
BOTANIQUE ET CRYPTOGAMIE, BIOLOGIE CELLULAIRE

CHIMIE ANALYTIQUE, QUALITOLOGIE ET NUTRITION

CHIMIE PHYSIQUE - PREVENTION DES RISQUES ET NUISANCES TECHNOLOGIQUES

CHIMIE THERAPEUTIQUE

CHIMIE ORGANIQUE PHARMACEUTIQUE HYDROLOGIE

PHARMACOGNOSIE, ETHNOPHARMACOLOGIE
Mme Anne FAVEL

Mme Joëlle MOULIN-TRAFFORT

Mme Catherine DEFOORT

$M$. Alain NICOLAY

Mme Estelle WOLFF

Mme Elise LOMBARD

Mme Camille DESGROUAS

M. Charles DESMARCHELIER

M. Pierre REBOUILLON

Mme Sandrine ALIBERT

Mme Caroline DUCROS

M. Marc MONTANA

Mme Manon ROCHE

Mme Fanny MATHIAS

M. Armand GELLIS

M. Christophe CURTI

Mme Julie BROGGI

$M$. Nicolas PRIMAS

$M$. Cédric SPITZ

M. Sébastien REDON

M. Riad ELIAS

Mme Valérie MAHIOU-LEDDET

Mme Sok Siya BUN

Mme Béatrice BAGHDIKIAN

\section{MAITRES DE CONFERENCE ASSOCIES A TEMPS PARTIEL (M.A.S.T.)}

CHIMIE ANALYTIQUE, QUALITOLOGIE ET NUTRITION

CHIMIE PHYSIQUE - PREVENTION DES RISQUES ET NUISANCES TECHNOLOGIQUES

DROIT ET ECONOMIE DE LA PHARMACIE

GESTION PHARMACEUTIQUE, PHARMACOECONOMIE ET ETHIQUE PHARMACEUTIQUE OFFICINALE, DROIT ET COMMUNICATION PHARMACEUTIQUES A L'OFFICINE ET GESTION DE LA PHARMAFAC
Mme Anne-Marie PENET-LOREC

M. Cyril PUJOL

M. Marc LAMBERT

Mme Félicia FERRERA

A.H.U.

CHIMIE ANALYTIQUE, QUALITOLOGIE ET NUTRITION

M. Mathieu CERINO

\section{ATER}




\section{DEPARTEMENT MEDICAMENT ET SECURITE SANITAIRE}

Responsable : Professeur Benjamin GUILLET

\section{PROFESSEURS}

PHARMACIE CLINIQUE

M. Stéphane HONORÉ

PHARMACODYNAMIE

M. Benjamin GUILLET

TOXICOLOGIE ET PHARMACOCINETIQUE

M. Bruno LACARELLE

Mme Frédérique GRIMALDI

M. Joseph CICCOLINI

\section{MAITRES DE CONFERENCES}

PHARMACODYNAMIE

PHYSIOLOGIE

TOXICOLOGIE ET PHARMACOCINETIQUE

TOXICOLOGIE GENERALE ET PHARMACIE CLINIQUE
M. Guillaume HACHE Mme Ahlem BOUHLEL

M. Philippe GARRIGUE

Mme Sylviane LORTET

Mme Emmanuelle MANOS-SAMPOL

Mme Raphaëlle FANCIULLINO Mme Florence GATTACECCA

M. Pierre-Henri VILLARD

Mme Caroline SOLAS-CHESNEAU

Mme Marie-Anne ESTEVE

\section{A.H.U.}

PHYSIOLOGIE / PHARMACOLOGIE

PHARMACIE CLINIQUE
Mme Anaïs MOYON

M. Florian CORREARD

\section{ATER.}


Mme Valérie AMIRAT-COMBRALIER, Pharmacien-Praticien hospitalier M. Pierre BERTAULT-PERES, Pharmacien-Praticien hospitalier Mme Marie-Hélène BERTOCCHIO, Pharmacien-Praticien hospitalier Mme Martine BUES-CHARBIT, Pharmacien-Praticien hospitalier M. Nicolas COSTE, Pharmacien-Praticien hospitalier Mme Sophie GENSOLLEN, Pharmacien-Praticien hospitalier M. Sylvain GONNET, Pharmacien titulaire Mme Florence LEANDRO, Pharmacien adjoint M. Stéphane PICHON, Pharmacien titulaire M. Patrick REGGIO, Pharmacien conseil, DRSM de I'Assurance Maladie Mme Clémence TABELE, Pharmacien-Praticien attaché Mme TONNEAU-PFUG, Pharmacien adjoint M. Badr Eddine TEHHANI, Pharmacien - Praticien hospitalier M. Joël VELLOZZI, Expert-Comptable 


\section{REMERCIEMENTS}

\section{Aux membres du jury,}

\section{A Monsieur le Professeur Pascal Rathelot, Président du jury}

Merci de me faire l'honneur de présider mon jury de soutenance de thèse mais également pour le savoir que tu m'as transmis durant mes stages et mes cours à la faculté de Marseille. J'ai beaucoup aimé travailler avec toi. Merci pour ta disponibilité et tes nombreux conseils durant mon internat.

\section{A Madame le Docteur Charlotte Papailhau, directrice de thèse}

Merci d'avoir accepté de diriger ce travail, pour ton implication, tes conseils, ta disponibilité et ta bonne humeur. J'ai beaucoup aimé travailler avec toi au Centre Gérontologique Départemental.

A Madame le Docteur Dorothée Naudet, membre du jury

Merci d'accepter d'être membre de mon jury de thèse, pour votre implication dans la conciliation médicamenteuse, votre professionnalisme, votre aide, votre partage et votre disponibilité. Mon semestre au Centre Gérontologique Départemental a été très intéressant en partie grâce à vous.

A Monsieur le Docteur Teddy Novais, membre du jury

Pour l'honneur que vous me faites en acceptant de juger ce travail, veuillez recevoir l'expression de ma sincère reconnaissance.

A toutes les personnes ayant contribuées à ce travail,

A Monsieur le Docteur Michel Grino, pour son aide dans ce travail, dans l'analyse statistique et sa disponibilité.

A Madame le Docteur Cristel Gille, pour son implication dans la conciliation médicamenteuse, sa sympathie. J'ai également beaucoup aimé travailler avec vous au SSR.

A Madame le Docteur Marie-Bénédicte Vincentelli, pour tout ce que vous avez pu m'apporter durant ce semestre qui fait partie de mes meilleurs stages malgré le contexte sanitaire qui nous a fortement impacté. Je vous remercie pour votre sympathie, votre bonne humeur et le savoir que vous avez pu me transmettre. 

A Madame le Docteur Catherine Molines, pour son aide et ses conseils à la réalisation de ce travail.

A Vincent Robert, pour son aide, ses conseils sur ce stage au Centre Gérontologique Départemental que j'ai beaucoup aimé et pour le super co-interne qu'il a été.

Aux pharmaciens que j’ai pu croiser durant mon internat :

Je vous remercie pour vos enseignements et votre partage.

Et particulièrement à :

- L'équipe de l'hôpital Nord : Madame le Docteur Martine Charbit, Madame le Docteur Colombini, Madame le Docteur Peyron et Monsieur le Docteur Castro

- L'équipe du CHU de Nice de stérilisation : Madame le Docteur Anne-Laure Chamorey et Madame le Docteur Claire-Hélène Nolin

Et au Docteur Coralie Lo Presti et au Docteur Elodie Bambina pour leurs nombreux conseils et partages durant mon semestre.

\section{Aux équipes hospitalières,}

Et plus particulièrement, à Angèle, cadre de santé au SCQIP, pour son implication, son partage et son soutien durant mes semestres.

\section{A ma famille,}

A ma mère, sans qui mes études n'auraient pas été possible ou du moins n'auraient pas été les mêmes. Merci pour ton soutien, ton aide et la confiance que tu as pu avoir en moi chaque jour. A mon père, pour son soutien, son aide et ses conseils durant mes études. Vous êtes des parents formidables qui ont toujours tout fait pour m'aider au mieux.

A ma famille qui a toujours cru en moi et qui m'a toujours soutenue.

A mes fidèles amies de l'internat et bien plus que ça, Flora et Wiem, nous avons révisé ensemble et réussi ensemble. A nos discussions interminables, nos révisions même après l'internat, nos voyages, nos escapades chez l'une et l'autre, nos trajets (même sur Lyon Wiwi pour le DU), je suis tellement heureuse de vous avoir rencontrées lors de mes études. L'internat n'aurait pas été pareil sans vous ! A Sandrine, pour nos moments passés ensemble, nos trajets et révisions. 

A Fanny Mathias, pour son soutien et ses nombreux conseils tout au long de mon internat.

A mes co-internes, avec qui j'ai passé de bons moments.

A mes amis de la faculté de Marseille, qui ont rendu ces années, ces révisions, ces soirées plus belles : Majda, Héloïse, Lisa, Anja (ma binôme), Julia, Polo, Eléonore, Antoine. Une mention spéciale à Majda qui m'a toujours soutenue et conseillée toutes ces années, merci d'être là pour moi.

A mes amis de Nice et d'ailleurs, Ilhame, Guillaume et Mohamed (" les 3 mousquetaires »), Gabriel, Christelle, Sophia, Rebecca, Yohann, Romain, Meriem et Charlène pour votre soutien malgré la distance.

A celui qui m'accompagne dans la vie de tous les jours, qui a rendu ma vie à Marseille plus belle qu'elle ne l'était déjà, merci pour ton soutien, ton aide, ta présence à mes côtés et ton amour. 

"L'Université n'entend donner aucune approbation, ni improbation aux opinions émises dans les thèses. Ces opinions doivent être considérées comme propres à leurs auteurs. " 


\section{TABLE DES MATIERES}

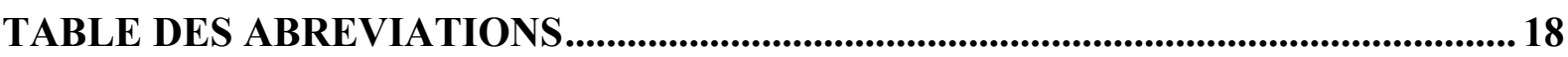

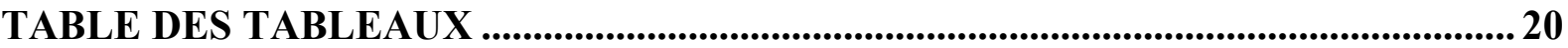

TABLE DES COMPLEMENTS ELECTRONIQUES ....................................................... 21

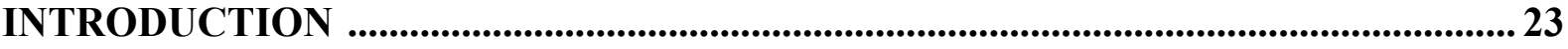

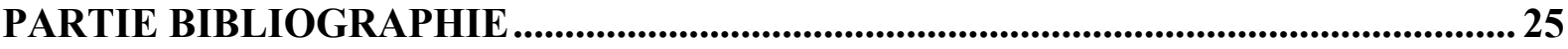

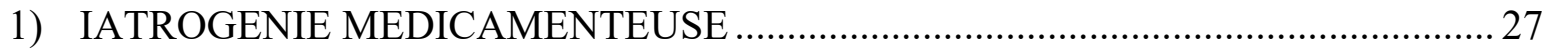

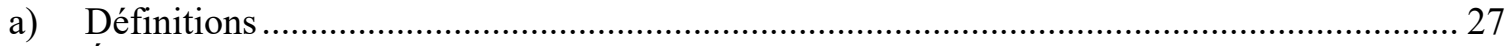

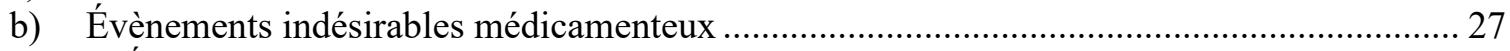

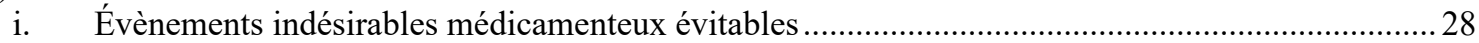

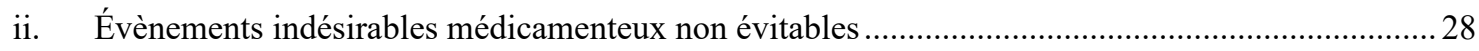

c) Risque iatrogène et prise en charge médicamenteuse du patient.......................................... 29

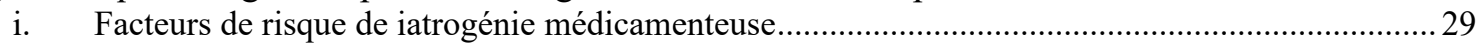

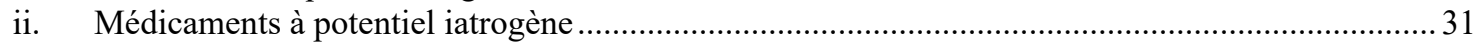

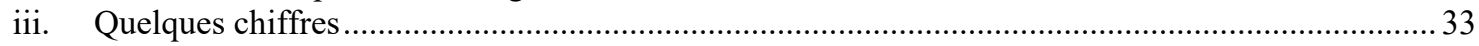

2) PRISE EN CHARGE MEDICAMENTEUSE DU SUJET AGE .................................. 33

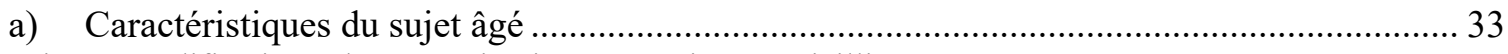

i. Modifications pharmacocinétiques associées au vieillissement..................................................... 33

ii. Modifications pharmacodynamiques associées au vieillissement....................................................... 35

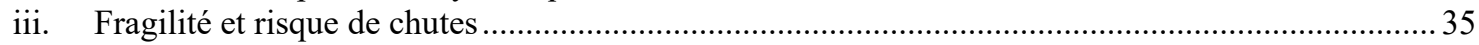

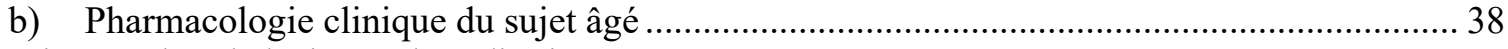

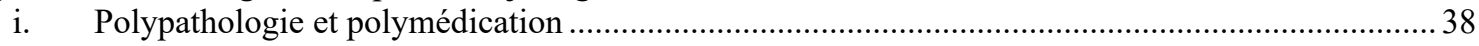

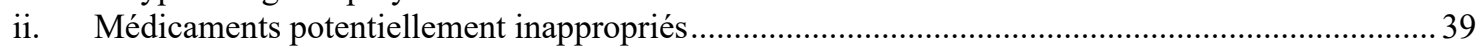

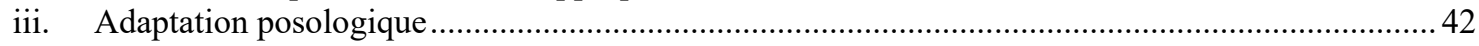

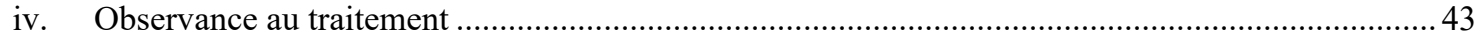

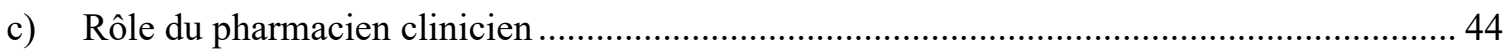

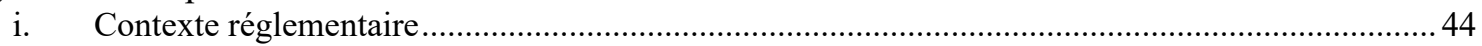

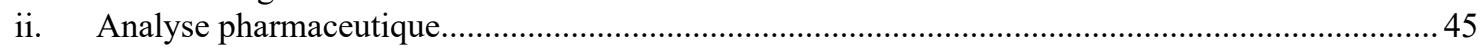

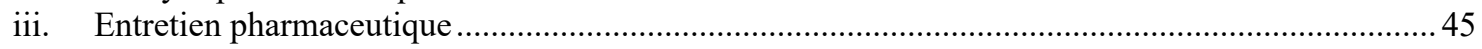

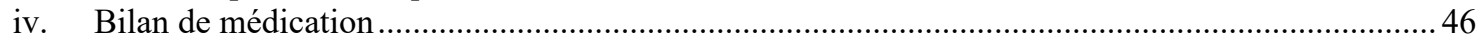

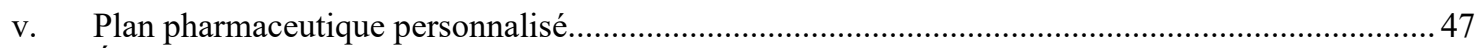

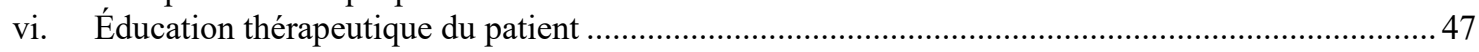

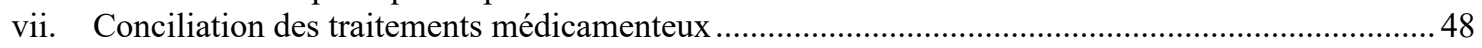

3) CONCILIATION DES TRAITEMENTS MEDICAMENTEUX …............................ 48

a) Définition de la conciliation des traitements médicamenteux ............................................... 48

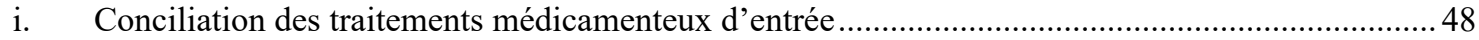

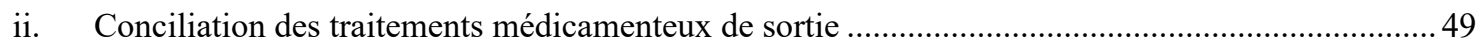

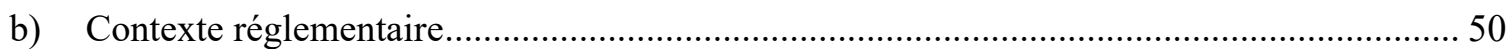

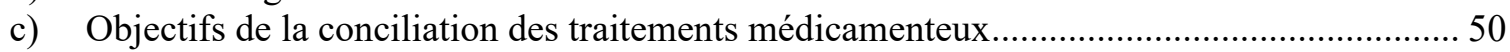

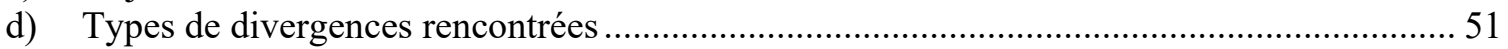

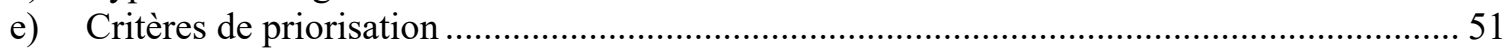

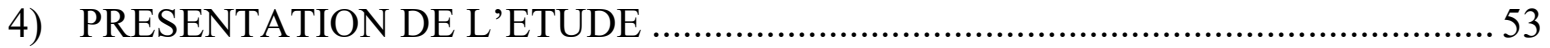

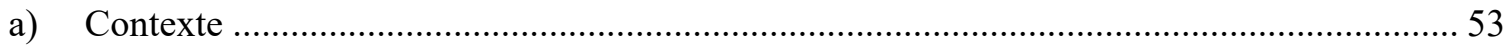

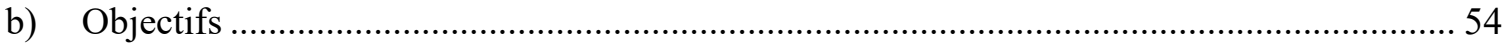

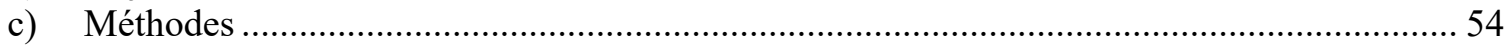

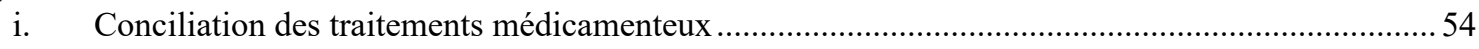

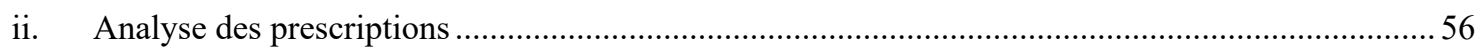

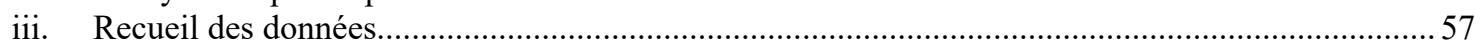

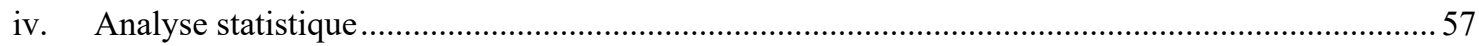


MATERIEL ET METHODES

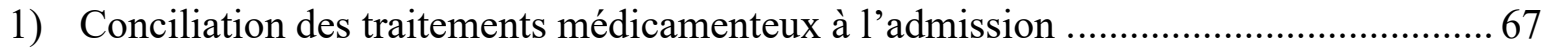

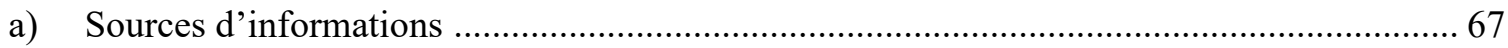

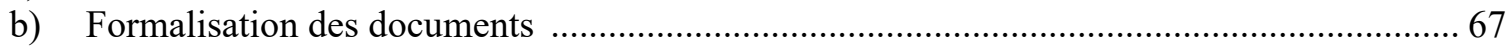

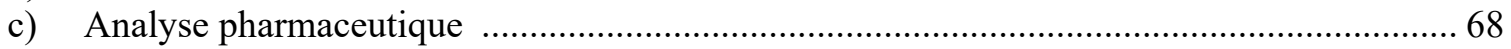

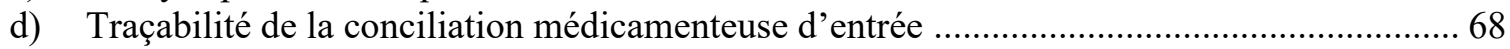

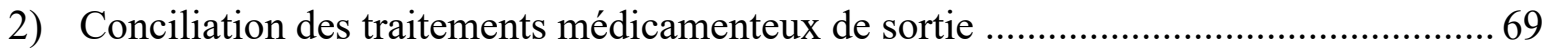

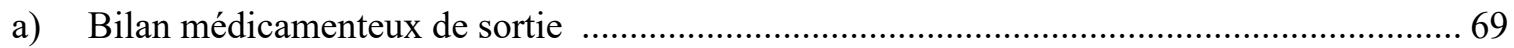

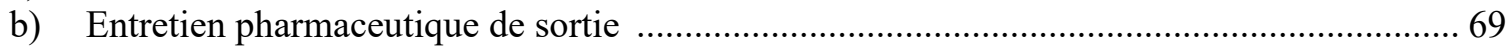

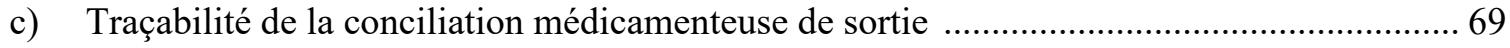

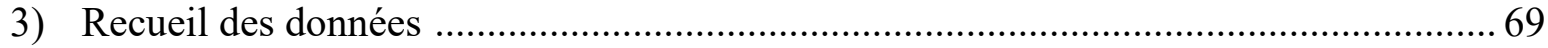

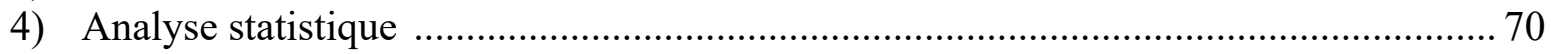

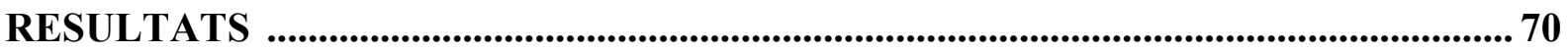

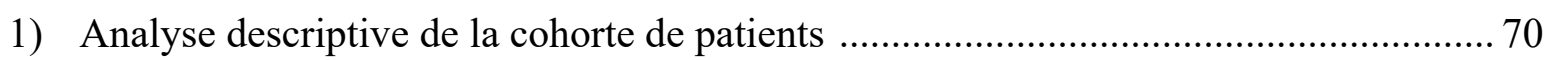

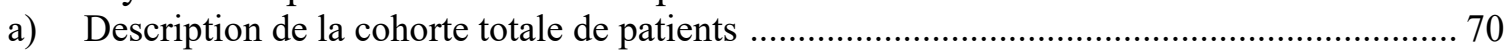

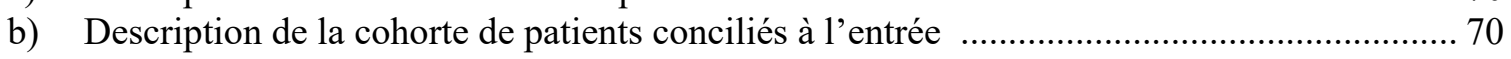

2) Conciliation des traitements médicamenteux à l'admission ......................................... 71

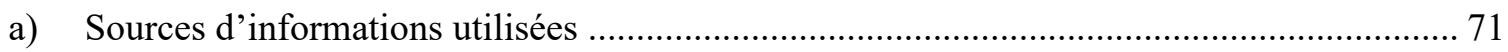

b) Divergences retrouvées lors des conciliations médicamenteuses d'entrée .......................... 72

c) Difficultés rencontrées lors de la conciliation médicamenteuse d'entrée ............................. 72

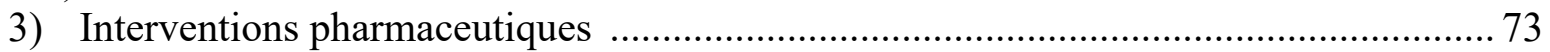

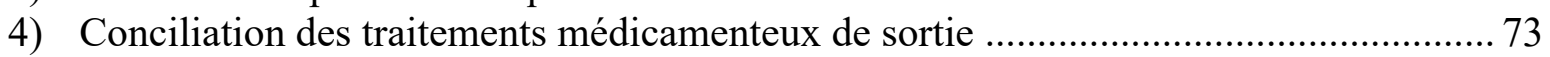

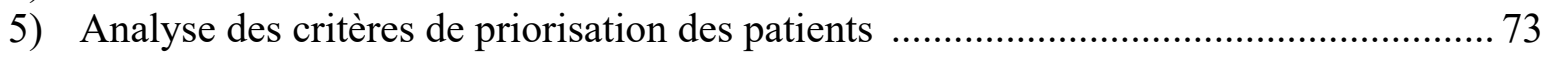

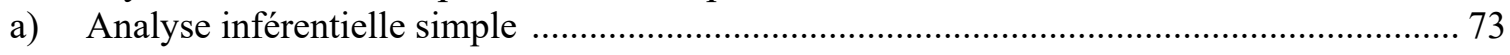

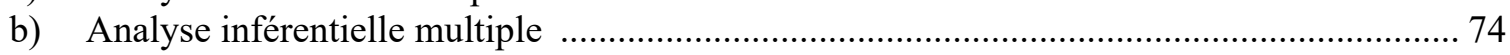

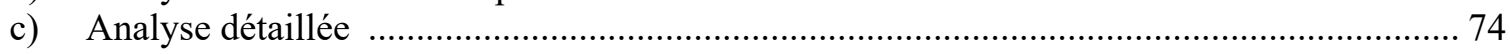

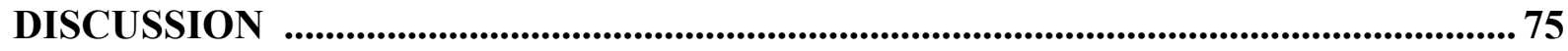

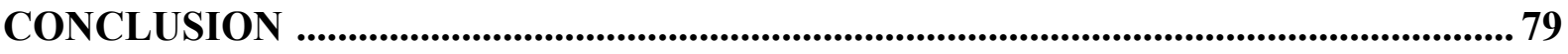

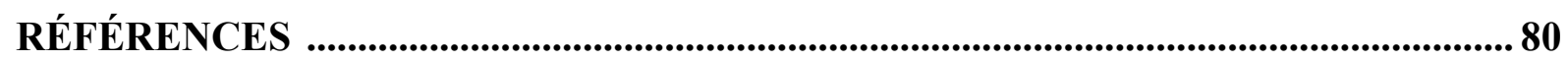

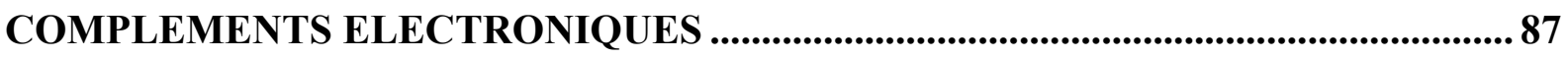

DISCUSSION GENERALE ET PERSPECTIVES .....................................................99

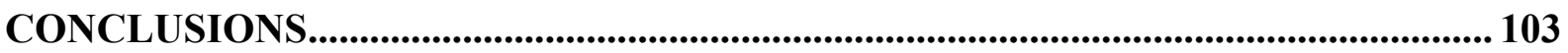

REFERENCES BIBLIOGRAPHIQUES …............................................................................. 107 


\section{TABLE DES ABREVIATIONS}

ADL : Activities of Daily Living

ADS : Anticholinergic Drugs Scale

AINS : Anti-Inflammatoires Non Stéroïdiens

AM : $\quad$ Assurance Maladie

AMM : Autorisation de Mise sur le Marché

ANSM : Agence Nationale de Sécurité du Médicament

ARA II : Antagonistes des Récepteurs de l'Angiotensine II

ARS : $\quad$ Agence Régionale de Santé

AVC : Accident Vasculaire Cérébral

AVK : Antivitamine K

BHE : Barrière Hémato-Encéphalique

BMO : Bilan Médicamenteux Optimisé

BMS : Bilan Médicamenteux de Sortie

CAQES : Contrat d'Amélioration de la Qualité et l'Efficience des Soins

CGD : Centre Gérontologique Départemental

Cl : $\quad$ Clairance rénale

CNIL : Commission Nationale de l'Informatique et des Libertés

COMEDIMS : Commission du Médicament et des Dispositifs Médicaux Stériles

CRPV : Centres Régionaux de Pharmacovigilance

CSA : $\quad$ Court Séjour Alzheimer

CSG : Court Séjour Gériatrique

CTM : Conciliation des Traitements Médicamenteux

DBI : $\quad$ Drug Burden Index

DFG : Débit de Filtration Glomérulaire

DGOS : Direction Générale d'Offre et des Soins

DI : $\quad$ Divergence Intentionnelle

DNI : $\quad$ Divergence Non Intentionnelle

DP : Dossier Pharmaceutique

DPI : $\quad$ Dossier Patient Informatisé

EHPAD : Etablissement d'Hébergement pour Personnes Agées Dépendantes

EI : $\quad$ Evènement Indésirable

EIG : Effet Indésirable Grave

EIM : Evènement Indésirable Médicamenteux

EM : $\quad$ Erreur médicamenteuse

ENEIS : Enquête Nationale sur les Evénements Indésirables liés aux Soins

EPP : Evaluation des Pratiques Professionnelles

ES : $\quad$ Etablissement de Santé

ET : $\quad$ Ecart-Type

ETP : Education Thérapeutique du Patient

FCT : $\quad$ Fiche de Conciliation des Traitements 
GPR : Guide de Prescription et Rein

HAD : Hospitalisation à Domicile

HAS : Haute Autorité de Santé

HDJ : Hospitalisation De Jour

IDE : Infirmier Diplômé d'Etat

IEC : Inhibiteurs de l'Enzyme de Conversion

IHC : Insuffisance Hépato-Cellulaire

IMC : Indice de Masse Corporelle

IP : Interventions Pharmaceutiques

IPP : Inhibiteurs de la Pompe à Protons

IR : Insuffisance Rénale

IRS : Insuffisance Rénale Sévère

ISRS : Inhibiteurs Sélectifs de la Recapture de la Sérotonine

JORF : Journal Officiel de la République Française

LAP : $\quad$ Logiciels d'Aide à la Prescription

MCO : $\quad$ Médecine Chirurgie Obstétrique

MPI : Médicaments Potentiellement Inappropriés

NCC MERP : National Coordinating Council for Medication Error Reporting and Prevention

OMA : Ordonnance Médicamenteuse à l'Admission

OMEDIT :Observatoire du Médicament, des Dispositifs médicaux et de l'Innovation Thérapeutique

OMS : Organisation Mondiale de la Santé

ORA : Odds Ratio Ajusté

OSH : Ordonnance de Sortie d'Hospitalisation

PA : $\quad$ Principe Actif

PACA : Provence-Alpes Côte d'Azur

PEC : $\quad$ Prise en Charge

PECM : Prise en Charge Médicamenteuse

PPP : $\quad$ Plan Pharmaceutique Personnalisé

PUI : $\quad$ Pharmacie à Usage Intérieur

RCP : $\quad$ Résumé des Caractéristiques du Produit

SIADH : Syndrome de sécrétion Inappropriée d'Hormone Anti-Diurétique

SMR : $\quad$ Service Médical Rendu

SNC : $\quad$ Système Nerveux Central

SSR : $\quad$ Soins de suite et de Réadaptation

STOPP/START : Screening Tool of Older Persons' Prescriptions/Screening Tool to Alert to Right Treatment

UCC : Unité Cognitivo-Comportementale

USLD : $\quad$ Unité de Soins de Longue Durée 


\section{TABLE DES TABLEAUX}

\section{PARTIE BIBLIOGRAPHIE}

Tableau 1 : Échelles d'évaluation de la fragilité du patient âgé

\section{PARTIE ARTICLE}

Tableau 1. Analyse descriptive de la cohorte de patients $(n=136$ patients $)$

Tableau 2. Analyse inférentielle simple

Tableau 3. Analyse inférentielle multiple

Tableau 4. Analyse détaillée 


\section{TABLE DES COMPLEMENTS ELECTRONIQUES}

Complément électronique 1 : Trame entretien patient et/ou famille

Complément électronique 2 : Fiche de conciliation des traitements à l'entrée

Complément électronique 3 : Bilan médicamenteux de sortie

Complément électronique 4 : Lettre explicative bilan médicamenteux de sortie jointe à la lettre de liaison d'hospitalisation 



\section{INTRODUCTION}

Les personnes âgées d'au moins 65 ans représentaient en 2018, 19,6\% de la population contre $19,2 \%$ un an auparavant et 18,8\% deux ans auparavant [1]. En 2020, le vieillissement de la population française se poursuit : plus d'une personne sur cinq en France a 65 ans ou plus [2]. En Europe, la part de cette population a également augmenté depuis une quinzaine d'années : elle est passée ainsi de $16,2 \%$ en 2003 à $19,7 \%$ en 2018 [2]. Ce type de patient est particulièrement exposé aux risques d'événements indésirables médicamenteux (EIM). En effet, les sujets âgés sont le plus souvent polypathologiques et donc polymédicamentés. La prescription médicamenteuse devient alors un problème de santé publique du fait de l'augmentation constante de la consommation de médicaments dans la population âgée. Le vieillissement physiologique et la fréquence des comorbidités modifient le rapport bénéfice/risque des médicaments : la connaissance et l'évaluation systématique de ces facteurs sont donc indispensables pour améliorer la qualité de la prescription médicamenteuse [3].

Dans cette optique, la Haute Autorité de Santé (HAS) impose d'étendre et de généraliser la conciliation des traitements médicamenteux (CTM) au sein des établissements de santé par le biais des certifications et notamment celle de 2020 (critère 2.2.1-06). Cette activité est également intégrée dans le Contrat d'Amélioration de la Qualité et l'Efficience des Soins (CAQES) ; établi entre l'organisme local d'Assurance Maladie (AM), l'Etablissement de santé (ES) et l'Agence Régionale de Santé (ARS) en matière de régulation des prescriptions médicamenteuses, de pertinence et de sécurité des soins [4]. La CTM est un processus interactif et pluriprofessionnel qui garantit la continuité des soins en intégrant à une nouvelle prescription les traitements en cours du patient [5]. L'enjeu principal est de prévenir ou corriger les EIM potentiellement iatrogènes, en favorisant la transmission d'informations complètes et exactes sur les médicaments du patient aux points de transition qui sont le plus souvent à risque médicamenteux tels que l'admission, la sortie et les transferts du patient [6].

Le pharmacien hospitalier se doit de mener des actions de pharmacie clinique au sein de l'ES. Parmi celles-ci sont retrouvées la réalisation de bilans de médication et les entretiens pharmaceutiques, avec notamment la CTM d'entrée et de sortie [7]. Des critères de priorisation à la réalisation de la CTM pour les patients hospitalisés ont été proposés par plusieurs OMEDIT [8-10] mais potentiellement non adaptés pour une population exclusivement gériatrique, ce qui est le cas précis de notre étude. 
En effet, dans cette population, tous les patients peuvent être considérés comme à risque si on s'intéresse notamment aux critères suivants : âge, polymédication, insuffisance rénale (IR) modérée, patient diabétique ou patient atteint de cancer. De ce fait, d'autres critères de priorisation ont été initialement définis en lien avec les équipes médicales de notre établissement. Cependant, ces choix de critères sont-ils pertinents ? En existeraient-ils d'autres à prioriser?

Ainsi, grâce à une étude prospective relevant les données des conciliations médicamenteuses d'entrée réalisées sur une période de 10 mois, l'objectif de ce travail était d'analyser et de définir des critères de priorisation pertinents pour la conciliation médicamenteuse chez une population exclusivement gériatrique. Ceci dans le but d'éviter le maximum d'erreurs médicamenteuses (EM) et de sensibiliser les patients et les professionnels de santé à l'intérêt de cette activité, aussi bien à l'entrée du patient qu'à sa sortie de l'établissement (dans le cadre du renforcement du lien ville-hôpital).

Dans ce mémoire, nous verrons dans un premier temps comment peut être définie la iatrogénie médicamenteuse et les évènements indésirables qui en découlent pour en prévenir le risque et optimiser la prise en charge médicamenteuse (PECM) spécifique des sujets âgés. Dans un second temps, nous nous focaliserons sur l'activité de conciliation médicamenteuse afin d'étudier et d'analyser les critères de priorisation possibles chez le patient âgé. Des compléments méthodologiques de notre étude principale seront détaillés avant de présenter l'article original, soumis à la Revue Thérapies. Enfin, après une discussion générale, nous évoquerons les différentes perspectives envisagées suite à ce travail. 
ETUDE OBSERVATIONNELLE DE FACTEURS PREDICTIFS D'ERREURS MEDICAMENTEUSES ET DE CRITERES DE PRIORISATION EN CONCILIATION MEDICAMENTEUSE GERIATRIQUE 



\section{1) IATROGENIE MEDICAMENTEUSE}

\section{a) Définitions}

L’Organisation Mondiale de la Santé (OMS) décrit en 1969 la iatrogénie médicamenteuse comme « Toute réponse néfaste et non recherchée à un médicament survenant à des doses utilisées chez l'homme à des fins de prophylaxie, de diagnostic et de traitement » [11]. Selon la HAS, elle correspond aux conséquences indésirables ou négatives sur l'état de santé individuel ou collectif de tout acte ou mesure pratiqués ou prescrits par un professionnel habilité et qui vise à préserver, améliorer ou rétablir la santé » [12].

On parle de iatrogénie médicamenteuse lorsque la thérapeutique médicamenteuse induit des effets, réactions, événements ou accidents indésirables, tant en raison des effets propres des médicaments concernés qu'à cause du contexte et des modalités de leur utilisation [13]. Elle représente un problème majeur de santé publique. En effet, la lutte contre la iatrogénie médicamenteuse est un des objectifs de la loi n 2004-806 du 9 août 2004 relative à la politique de santé publique. En 2015, elle est toujours parmi les axes identifiés par la loi de financement de la sécurité sociale [13,14].

\section{b) Évènements indésirables médicamenteux}

Un événement indésirable médicamenteux (EIM) peut se qualifier comme « un dommage survenant chez le patient, lié à sa PECM et résultant de soins appropriés, de soins inadaptés ou d'un déficit de soins » [15]. L'EIM peut engendrer l'aggravation de la pathologie existante, l'absence d'amélioration attendue de l'état de santé, la survenue d'une pathologie nouvelle ou prévenue, l'altération d'une fonction de l'organisme, une réaction nocive due à la prise d'un médicament [15].

Parmi ces EIM, on retrouve deux catégories : les EM ayant une conséquence clinique liée à un dysfonctionnement dans le circuit de la PECM d'un patient (EIM évitables); et les effets indésirables qui sont liés à la pharmacologie du médicament et qui sont potentiellement connus par le patient (EIM non évitables) [16]. 
«Un effet indésirable grave (EIG) est un effet indésirable létal, ou susceptible de mettre la vie en danger, ou entraînant une invalidité ou une incapacité importantes ou durables, ou provoquant ou prolongeant une hospitalisation, ou se manifestant par une anomalie ou une malformation congénitale » [17]. Les EIG peuvent être à l'origine d'une hospitalisation. Une part non négligeable de ces EIG serait liée aux médicaments et serait considérée comme évitable $[18,19]$.

\section{i. Évènements indésirables médicamenteux évitables}

L'EIM évitable peut se désigner comme tout événement indésirable qui ne serait pas survenu si les soins avaient été conformes à la prise en charge (PEC) considérée comme satisfaisante au moment de la survenue de cet événement $[15,18]$. Il est lié à un dysfonctionnement non intentionnel dans l'organisation des soins. L'EM est évitable car elle manifeste ce qui aurait dû être fait et qui ne l'a pas été au cours de la PECM d'un patient. Lorsqu'un EIM s'avère secondaire à une EM, il est considéré comme évitable ; en particulier lorsqu'il s'agit d'un effet indésirable $[15,20]$.

De tels évènements peuvent résulter de plusieurs pratiques notamment : la prescription, la communication des ordonnances, l'approvisionnement, la préparation, la délivrance, l'administration, l'information et l'éducation du patient, le suivi thérapeutique et les modalités d'utilisation. Un référentiel répertoriant les EM selon différents critères a été proposé dès 1995 par le National Coordinating Council for Medication Error Reporting and Prevention (NCC MERP). Les critères qui avaient été retenus pour caractériser et qualifier à posteriori l'EM étaient : sa nature, son type, l'étape de réalisation dans la chaine de soins, les causes, le niveau de réalisation de l'erreur et la gravité de ses conséquences cliniques pour le patient [21,22].

\section{ii. Évènements indésirables médicamenteux non évitables}

Ils correspondent aux effets indésirables médicamenteux et surviennent dans des conditions normales d'utilisation du produit. Ils sont généralement connus et prévisibles étant liés aux propriétés pharmacologiques du médicament [23]. 
Selon l'OMS, un effet indésirable est une « réaction nocive et non voulue à un médicament, se produisant aux posologies normalement utilisées chez l'homme pour la prophylaxie, le diagnostic ou le traitement d'une maladie, ou pour la restauration, la correction ou la modification d'une fonction physiologique, ou résultant d'un mésusage du médicament ou produit » [15]. Ces effets indésirables font l'objet de déclarations auprès des Centres Régionaux de Pharmacovigilance (CRPV).

c) Risque iatrogène et prise en charge médicamenteuse du patient

\section{i. $\quad$ Facteurs de risque de iatrogénie médicamenteuse}

Les facteurs de risque iatrogènes sont liés à l'âge du patient, à une polypathologie, à une mauvaise utilisation des médicaments, aux médicaments eux-mêmes avec la polymédication et les interactions médicamenteuses, au manque de coordination entre les différents prescripteurs [24] ainsi qu'au contexte socio-environnemental [25]. La présence de troubles cognitifs, de pathologies de la mémoire et des troubles de la compréhension entraîne également une adhésion plus complexe au traitement [26].

- Age :

L'âge et la polypathologie sont des facteurs de gravité des accidents iatrogéniques [27].

Lors du vieillissement, l'organisme subit des modifications au niveau des paramètres pharmacocinétiques et pharmacodynamiques des médicaments (que nous verrons dans une prochaine partie), ayant d'une part des conséquences sur l'action d'un certain nombre de médicaments et d'autre part, sur leur administration.

De nombreux facteurs sont susceptibles d'interférer avec l'administration et le bon usage des médicaments [25] :

- La diminution des capacités physiques,

- Les difficultés de communication,

- Les troubles de la déglutition (risque de stagnation prolongée des médicaments dans la bouche et l'œsophage),

- La diminution de l'acuité visuelle ou de l'audition. 
Dans certains cas, il n'existe plus de possibilité de compensation à ces facteurs pouvant entrainer une mauvaise observance au traitement, la seule solution devient alors la prise contrôlée par un tiers tels que la famille, un proche ou un infirmier diplômé d'état (IDE) à domicile.

- Mauvaise utilisation des médicaments :

Plusieurs situations peuvent entraîner cette situation [25] :

- Une prescription inadaptée : par les objectifs thérapeutiques, par des prescriptions non pertinentes au vu de l'indication/du choix de la classe médicamenteuse/de la dose et/ou de la durée, interactions médicamenteuses, association de médicaments comportant des effets indésirables communs et majorant leur toxicité, surveillance inadaptée, réévaluation de la prescription insuffisante, médicaments inutiles ;

- Une information insuffisante du patient et de son entourage ;

- Une automédication inappropriée ;

- Une mauvaise observance du traitement.

- Manque d'étude chez le sujet âgé :

Lors de l'obtention d'une autorisation de mise sur le marché (AMM), les essais thérapeutiques d'un nouveau médicament chez les personnes âgées ne concernent bien souvent que des effectifs relativement réduits. Il n'y a souvent pas d'essais thérapeutiques spécifiques au sujet âgé et une transposition de conclusions d'études est faite chez le sujet plus jeune dans cette population [28].

- Polymédication :

Les EI sont favorisés par la polymédication en réponse à une polypathologie fréquente. Les effets indésirables sont plus fréquents lorsque la consommation médicamenteuse est plus importante. 
De nombreux facteurs prédisposent à la polymédication chez le sujet âgé [28] :

- Les multiples prescriptions

- La tendance des prescripteurs à prescrire

- La demande des patients

- La prescription pour des symptômes et non une maladie

- Le renouvellement automatique des prescriptions

- L'automédication et l'auto-prescription.

La prise en compte de l'ensemble de ces facteurs de risque au moment de l'instauration, de la surveillance ou de la poursuite d'un traitement médicamenteux, permettra de diminuer la survenue d'EI [25].

- Contexte socio-environnemental :

Ces facteurs peuvent influencer la PECM ainsi que le suivi thérapeutique, comme l'isolement social ou géographique, la dépendance, le changement du mode de vie (déménagement, institutionnalisation) ou les conditions climatiques extrêmes [25].

\section{ii. Médicaments à potentiel iatrogène}

Du fait de la morbidité rencontrée dans cette tranche d'âge, la plupart des médicaments prescrits et administrés sont des substances à index thérapeutique étroit et exposent à une toxicité dosedépendante (psychotropes, antihypertenseurs, digoxine, anti-inflammatoires, anticoagulants) [29]. Les médicaments à demi-vie longue sont également à risque plus important d'EI chez la personne âgée.

Quelques exemples d'EI sont fréquemment retrouvés chez le sujet âgé [30] :

- L'hypotension artérielle due aux associations fréquentes d'antihypertenseurs, aux dérivés nitrés, aux neuroleptiques et aux opioïdes, 
- L'IR aiguë retrouvée avec les diurétiques, les anti-inflammatoires non stéroïdiens (AINS), les inhibiteurs de l'enzyme de conversion (IEC) ou antagonistes des récepteurs de l'angiotensine II (ARA II), les aminosides et les produits de contraste,

- Les troubles du rythme et/ou de la conduction cardiaque retrouvés avec les digitaliques, les bêtabloquants, les antiarythmiques, les inhibiteurs calciques et les médicaments responsables de troubles électrolytiques,

- Les troubles neurologiques (troubles de la vigilance, la confusion, la démence...) retrouvés avec les anxiolytiques, les antidépresseurs, les neuroleptiques, les antiparkinsoniens, les opioïdes, les antibiotiques tels que les quinolones et les anticholinergiques,

- Les accidents hémorragiques pouvant être retrouvés avec les anticoagulants surtout si association avec des antiagrégants plaquettaires ou des AINS,

- Les hypoglycémies dues à l'insuline ou aux sulfamides hypoglycémiants, plus ou moins en association avec des médicaments altérant les mécanismes de contre-régulation,

- Les ulcérations gastroduodénales avec les AINS.

Une surveillance de l'état clinique des patients, une surveillance de leurs paramètres biologiques ainsi que des dosages plasmatiques des principes actifs des médicaments s'avèrent justifiés et primordiaux quant à la PEC optimale de ces patients fragilisés.

Deux classes thérapeutiques souvent prescrites sont à haut risque iatrogène dans cette population : les psychotropes (risque de chutes, fractures et troubles confusionnels [31]) et les anticholinergiques (risque de confusions, chutes et rétentions urinaires). Pour ces derniers, ces effets sont plus marqués chez le sujet âgé à cause de l'affaiblissement du système cholinergique induit par le vieillissement [32].

La polymédication, ainsi que les troubles neuropsychiatriques du sujet âgé doivent donc rendre le prescripteur de psychotropes et d'anticholinergiques particulièrement vigilant dans cette population vulnérable [33-35]. L'usage des listes de médicaments potentiellement inappropriés (MPI) que nous développerons plus tard pourrait permettre d'identifier les médicaments au rapport bénéfice risque défavorable chez les sujets âgés et d'orienter le choix du prescripteur vers des traitements plus favorables au patient [31]. 


\section{iii. Quelques chiffres}

Chez la personne âgée, la iatrogénie médicamenteuse représente jusqu'à $20 \%$ des hospitalisations aux urgences des plus de 75 ans et $25 \%$ des admissions des plus de 85 ans [36]. Les EM peuvent survenir tout au long du processus de PECM du patient [37]. Certaines études citent les erreurs de prescription comme les plus fréquemment impliquées dans la survenue des EM [38-40]. Les erreurs sont plus fréquentes à l'admission, indépendamment du type de service [41], notamment dans une étude de Bobb et al., il a été retrouvé 2,4 erreurs pour 1000 ordonnances dont $64 \%$ lors de l'admission [42].

Concernant la typologie des erreurs, les erreurs de doses figurent parmi les plus fréquentes [4244]. Les erreurs d'omission (53,0 \%) sont celles survenant le plus fréquemment à l'étape de la prescription, suivies des erreurs de dosage $(15,5 \%)$, de fréquence $(8,5 \%)$ et de voie d'administration (5,0\%) [45]. D'autres erreurs sont également rencontrées telles que la présence d'interactions médicamenteuses, la non prise en compte des allergies du patient, la prescription d'un médicament non indiqué, un défaut dans le suivi thérapeutique, une confusion dans l'identité du patient ou une non-conformité réglementaire de la prescription.

L'ensemble de ces éléments introduit une partie de la complexité de la PECM du sujet âgé que nous allons détailler à présent.

\section{2) PRISE EN CHARGE MEDICAMENTEUSE DU SUJET AGE}

a) Caractéristiques du sujet âgé

\section{i. Modifications pharmacocinétiques associées au vieillissement}

La pharmacocinétique signifie «ce que l'organisme fait au médicament ». Elle se définit comme l'étude du devenir du médicament dans l'organisme [28].

Le vieillissement peut avoir des conséquences sur l'action des médicaments en considérant les paramètres pharmacocinétiques : 
- La réduction de la fonction rénale : la posologie des médicaments à élimination rénale doit être adaptée à l'estimation du débit de filtration glomérulaire (DFG) [25]. Avec l'âge, il existe une diminution du poids des reins (cortex) et du nombre de glomérules. Les modifications fonctionnelles constatées sont alors une diminution du flux sanguin rénal, de la filtration glomérulaire avec une importante variabilité, de la sécrétion et réabsorption tubulaires et de l'excrétion rénale des médicaments à élimination rénale prédominante engendrant une IR chronique " physiologique ». De nombreux médicaments ont donc une demi-vie augmentée chez le sujet âgé [28].

- L'hypoprotidémie et l'hémoconcentration chez les patients dénutris : il existe un risque potentiel de surdosage des médicaments fortement liés aux protéines plasmatiques [25]. La distribution des médicaments peut être modifiée avec l'âge par la diminution de l'albumine et l'augmentation de la fraction libre [46].

- Les modifications de la composition corporelle soit la perte en eau corporelle, au niveau ostéo-musculaire et le gain adipeux : les distributions masse grasse/ masse maigre et donc les volumes de distributions sont modifiés (augmentation du pourcentage de masse grasse et diminution de la masse maigre). Il y a une réduction des débits sanguins des organes et tissus. Des conséquences sur la distribution des médicaments sont notables telles que l'augmentation du volume de distribution des médicaments liposolubles, une diminution du volume de distribution des médicaments hydrosolubles, un allongement de la phase de distribution et une distribution préférentielle des médicaments vers le cerveau [28].

- Des variations de la réponse aux médicaments : notamment ceux qui agissent sur le système nerveux central (SNC) (plus grande sensibilité à tout effet neurologique des médicaments) et sur l'appareil cardiovasculaire [46]. La modification de la perméabilité de la barrière hémato-encéphalique (BHE) peut entrainer une plus grande sensibilité aux médicaments agissant au niveau du SNC (effet sédatif par exemple) [25].

- Une diminution du métabolisme hépatique de certains médicaments dépendants du flux hépatique et des cytochromes P450 [46] : le volume et le poids du foie diminuent ainsi que le nombre d'hépatocytes et le débit sanguin hépatique [28].

- Une diminution du pH gastrique, du débit splanchnique, de la mobilité intestinale et un ralentissement de la vidange gastrique ont des conséquences sur la résorption digestive notamment avec un ralentissement de la vitesse de résorption des médicaments avec un pic plasmatique plus tardif [28]. 
Pour de nombreux médicaments, ces variations pharmacologiques liées à l'âge sont modérées et ne justifient pas nécessairement une modification des doses [46]. En revanche, l'utilisation de certains médicaments est à adapter à chaque patient, notamment à leur fonction rénale (estimation systématique du DFG) et à leur état de nutrition (albuminémie).

Cependant, certains médicaments ne devraient pas être prescrits chez le patient âgé, du fait d'une balance bénéfice/risque défavorable comparés à d'autres alternatives thérapeutiques [46].

ii. Modifications pharmacodynamiques associées au vieillissement

La pharmacodynamie signifie « ce que le médicament fait à l'organisme ».

Le vieillissement peut aussi avoir des conséquences sur l'action des médicaments en considérant cette fois-ci la pharmacodynamie des médicaments [25] :

- Le vieillissement du cœur, tel que la perte du contingent de cellules nodales, pouvant entraîner une plus grande sensibilité à certains médicaments (troubles voire blocs conductifs) ;

- La fragilité osseuse nécessitant de surveiller particulièrement le risque d'hypotension orthostatique favorisé par certains médicaments (chutes, fractures).

Ces modifications physiologiques co-existent souvent avec de multiples pathologies qui sont aggravées par des épisodes aigus intercurrents (déshydratation, décompensation cardiaque, maladies infectieuses...) [25]. Ces épisodes aigus intercurrents (et leurs conséquences comme par exemple une IR) expliquent que même des médicaments pris au long cours peuvent être à l'origine d'un accident médicamenteux [25].

\section{iii. Fragilité et risque de chutes}

Le vieillissement est un processus physiologique continu et variable selon les individus. Le terme fragilité décrit la réduction multisystémique des réserves fonctionnelles apparaissant chez certaines personnes âgées et limitant alors les capacités de leur organisme à répondre au stress même mineur. Cet état d'instabilité physiologique expose l'individu à de multiples risques tels qu'à une décompensation fonctionnelle, à une perte d'autonomie, d'institutionnalisation et au décès [47]. 
L'étude de Fried et al. définit cinq caractéristiques de " phénotype de fragilité » : faiblesse, faible endurance, activité physique réduite, vitesse de marche lente et perte de poids involontaire au cours de la dernière année. Les personnes présentant au moins trois de ces caractéristiques sont qualifiées de « fragiles » tandis que les personnes « préfragiles » n'en présentent qu'une ou deux [48]. Cette étude a permis de démontrer que les personnes considérées fragiles (selon leurs critères) étaient plus à risque de développer des limitations fonctionnelles et des déficiences au niveau de la mobilité, de chuter, d'être hospitalisées et de décéder dans un délai de trois ans $[48,49]$.

Il existe aujourd'hui de nombreuses échelles d'évaluation de la fragilité du patient âgé selon l'approche envisagée [50] (Tableau 1).

\section{Tableau 1 : Échelles d'évaluation de la fragilité du patient âgé}

\begin{tabular}{|c|c|c|}
\hline Type d'approche & Nom du test & Critères du test \\
\hline \multirow{4}{*}{ Motrice } & Critères de Fried & $\begin{array}{l}\text { - Perte de poids involontaire } \\
\text { - Vitesse de marche lente } \\
\text { - Faible endurance } \\
\text { - Faiblesse/fatigue } \\
\text { - Activités physiques réduites }\end{array}$ \\
\hline & Vitesse de marche & Mètre/ secondes \\
\hline & $\begin{array}{c}\text { Score SPPB } \\
\text { (Short Physical Performance Battery) }\end{array}$ & $\begin{array}{l}\text { - Test d'équilibre } \\
\text { - Vitesse de marche } \\
\text { - Test de lever de chaise }\end{array}$ \\
\hline & $\begin{array}{c}\text { TUG test } \\
\text { (Timed Up and Go) }\end{array}$ & $\begin{array}{l}\text { Se lever d'une chaise avec accoudoir, marcher sur trois } \\
\text { mètres, faire demi-tour et revenir s'assoir }\end{array}$ \\
\hline $\begin{array}{l}\text { Médico-psycho- } \\
\text { sociale }\end{array}$ & $\begin{array}{l}\text { Echelle de Rockwood Clinical Frailty } \\
\text { Scale }\end{array}$ & $\begin{array}{l}\text { - Mobilité } \\
\text { - ADL (Activities of Daily Living, autonomie) } \\
\text { - Incontinence } \\
\text { - Troubles cognitifs }\end{array}$ \\
\hline \multirow{2}{*}{$\begin{array}{l}\text { Outil de repérage } \\
\text { Dépistage }\end{array}$} & Questionnaire Prisma 7 & $\begin{array}{l}\text { - Age }>85 \text { ans } \\
\text { - Sexe masculin } \\
\text { - Limitation des activités } \\
\text { - Sédentarisme } \\
\text { - Aide d'une personne proche } \\
\text { - Utilisation d'une canne, d'un déambulateur ou d'un } \\
\text { fauteuil roulant }\end{array}$ \\
\hline & $\begin{array}{l}\text { Questionnaire du Gérontopôle de } \\
\text { Toulouse }\end{array}$ & $\begin{array}{l}\text { - Vie seule à domicile } \\
\text { - Perte de poids au cours des } 3 \text { derniers mois } \\
\text { - Fatigue depuis les } 3 \text { derniers mois } \\
\text { - Difficulté pour se déplacer } \\
\text { - Plainte de mémoire } \\
\text { - Vitesse de marche ralentie }\end{array}$ \\
\hline $\begin{array}{l}\text { Évaluation de la } \\
\text { Précarité }\end{array}$ & $\begin{array}{c}\text { Score EPICES } \\
\text { (Évaluation de la Précarité et des } \\
\text { Inégalités de santé dans les } \\
\text { Centres d'examens de Santé) }\end{array}$ & Autoquestionnaire (11 critères) \\
\hline
\end{tabular}


Concernant le risque de chute, il n'est pas en rapport avec l'âge chronologique. En effet, les capacités d'adaptation au risque déclinent régulièrement avec l'avancée en âge, de la personne âgée active et dynamique à la personne âgée dépendante. De plus, des facteurs intrinsèques tels que les médicaments ou les maladies altérant les fonctions sensitives, cognitives ou motrices ou bien extrinsèques tels que les facteurs comportementaux ou environnementaux peuvent favoriser la chute.

La prévalence des chutes est importante et peut occasionner une forte morbi-mortalité chez :

- La personne âgée fragile ayant une réduction de ses capacités d'adaptation qui ne lui permet plus de faire face à des perturbations externes mineures. Toute chute lui fait courir un risque majeur de perte d'autonomie et de désinsertion sociale, et éventuellement une institutionnalisation.

- La personne âgée dépendante vivant en institution ayant un risque de chute particulièrement élevé, aux conséquences graves [51].

Ces chutes multifactorielles, sont avant tout des marqueurs de mauvais état de santé. Elles sont la conséquence de l'affaiblissement général, des démences ou d'autres (poly)pathologies, des polymédications, parfois des contentions [51]. Celles qui surviennent à domicile sont un signe précurseur de la perte d'autonomie et représentent un problème majeur de santé publique.

Lorsque la chute est responsable de fracture, il s'agit majoritairement de cas de fracture du col du fémur, indirectement responsable d'une mortalité élevée dans les mois qui suivent. Le risque fracturaire est plus important en cas de pathologies fragilisant l'os, altérant la vision, la marche et l'équilibre ou en cas de polypathologie chronique.

Par ailleurs, l'impact psychologique de la chute peut être majeur. Le syndrome post-chute se caractérise par une désadaptation psychomotrice par diminution spontanée de l'activité ainsi que des capacités fonctionnelles, troubles posturaux (telle que la rétropulsion) et troubles de la marche, favorisant ainsi le risque de nouvelle chute. Sa prise en charge, réadaptative et psychosociale, est essentielle au maintien de l'autonomie de la personne âgée [51]. 
b) Pharmacologie clinique du sujet âgé

\section{i. Polypathologie et polymédication}

Il n'existe pas de définition consensuelle de la polypathologie, cependant ce terme est couramment utilisé pour parler d'une accumulation de pathologies. On parlera de polypathologie lorsque les patients souffrent d'au moins deux maladies chroniques [52].

Elle est souvent associée à :

- Une vulnérabilité sociale et psychique,

- Une perte d'autonomie,

- Une altération de la qualité de vie,

- Une dépression,

- Ou une déficience sensorielle.

La prévalence des maladies chroniques augmente du fait de l'allongement de l'espérance de vie [53].

Précédemment abordée comme facteur de risque de iatrogénie médicamenteuse, la polypathologie peut être à l'origine d'une polymédication qui augmente d'une part le risque d'EI directement liés à chaque médicament mais aussi dus aux interactions médicamenteuses.

Selon l'OMS, la polymédication correspond à « l'administration de nombreux médicaments de façon simultanée ou par l'administration d'un nombre excessif de médicaments », fréquemment observée chez les personnes âgées [54]. Elle est facilitée par le nomadisme médical, les prescripteurs multiples qui s'ignorent, la superposition de traitements symptomatiques sans pertinence et une demande parfois insistante de traitement par le patient $[55,56]$.

La définition classique se réfère à la prise régulière d'au moins 5 médicaments [52] mais il n'existe pas de seuil consensuel. En effet, plusieurs seuils de définition de la polymédication peuvent être rencontrés dans la littérature (supérieur à 5, supérieur à 8 , supérieur à 10 ou seulement la notion de " nombreux médicaments »). Mais également, plusieurs auteurs définissent la polymédication par l'utilisation de 5 médicaments ou plus [57,58], du fait de la croissance linéaire du risque d'effets indésirables avec le nombre de médicaments et ce à partir de 5 médicaments [54,59]. 
En France, selon les données de l'AM, en 2015, $30 \%$ à $40 \%$ des personnes âgées de 75 ans ou plus prennent au moins 10 médicaments différents par jour [60]. La gestion de la polymédication est donc un enjeu majeur de santé publique, particulièrement pour les médecins généralistes mais également en secteur hospitalier lors de la réévaluation des prescriptions. Des analyses doivent systématiquement être faites grâce à des logiciels d'aide à la prescription (LAP) tel que Thériaque [61] ou bien DDI Predictor [62]. En effet, ce dernier permet de prédire quantitativement l'impact sur l'exposition des médicaments des interactions médicamenteuses d'origine métabolique (menées par les cytochromes P450 3A4, 2D6, 2C9, 2 C19 et 1A2).

\section{ii. Médicaments potentiellement inappropriés}

Pour de multiples raisons, les personnes âgées ont un risque plus important d'être victimes d'un EIM du fait d'une sensibilité accrue à l'action des médicaments. La prescription du traitement le plus adapté pour obtenir le meilleur rapport bénéfice/risque est un défi de tous les jours pour le praticien du patient âgé.

Certains médicaments augmenteraient le risque d'EI et sont donc considérés comme potentiellement inappropriés (« potentially inappropriate medications ») dans cette population. Ainsi, ce type de médicament est défini comme un médicament ayant un rapport bénéfice/risque défavorable et/ou une efficacité discutable par rapport à d'autres alternatives thérapeutiques plus sûres [63].

La prescription chez les sujets âgés n'est pas toujours optimale [64]:

- Le « misuse » ou prescription inappropriée est le concept le plus étudié. Les médicaments sont indiqués mais ne sont pas correctement prescrits (mauvaise fréquence, dose, durée, mauvais suivi...) ou la balance bénéfice/risque est défavorable. Ceci correspond aux MPI dont une liste a été établie initialement par Beers [65].

- L'« overuse » ou excès de prescription : lorsque des médicaments n'ayant pas d'indication valide ou présentant une efficacité discutable sont prescrits. Un médicament inefficace étant un médicament avec un service médical rendu (SMR) insuffisant. 
- L'« underuse » ou défaut de prescription : lorsque des médicaments indiqués et efficaces ne sont pas prescrits. Cette sous-utilisation de médicaments est le résultat de la faible représentation de la population gériatrique et/ou polypathologique lors des essais cliniques, ne permettant pas d'évaluer correctement le rapport bénéfice/risque du médicament. Les études post-AMM et la pharmacovigilance prennent ainsi toute leur importance [66].

Différentes listes et méthodes de relecture d'ordonnances existent pour identifier ces concepts de prescription sous-optimale. Les listes utilisent des critères généraux, elles sont dites explicites. Les méthodes, quant à elles, utilisent les listes et détaillent leur application aux particularités des patients, elles sont dites implicites [67].

Aux Etats-Unis, en 1991, un consensus d'experts liste les médicaments à éviter chez les personnes âgées pour limiter la survenue d'effets indésirables : ce sont les critères de Beers [65]. Une liste de 30 médicaments ou classes médicamenteuses avait été élaborée, indépendamment des doses mais tenant compte de la posologie et de la durée de prescription. Cette liste est dite « explicite » car elle est indépendante des caractéristiques des patients.

En 1997, de nouveaux critères de Beers ont été établis. En effet, de nouveaux médicaments étaient apparus sur le marché et les connaissances sur les effets indésirables chez la personne âgée se multipliaient. La nouvelle liste proposait 28 médicaments ou classes médicamenteuses applicables aux personnes âgées de 65 ans et plus, et 35 médicaments dans 15 indications médicales connues [63]. Cette liste a été également réactualisée en 2003 [68]. En 2001, une variante a été proposée en classant les médicaments en trois catégories : médicaments à éviter systématiquement (11 médicaments), médicaments rarement appropriés (huit médicaments), médicaments parfois indiqués mais souvent mal utilisés (14 médicaments) [69].

Au Canada, une autre liste de médicaments inappropriés a été proposée par McLeod et al. en raison d'un désaccord sur l'inclusion de certains médicaments par Beers ; en introduisant notamment la notion d'interactions maladie/médicament et médicament/médicament [70]. Ces trois paramètres (médicaments, interactions maladie/médicament et médicament/médicament) caractérisent l'ensemble des critères qualifiant la prescription inappropriée en gériatrie. Ces critères ont été déterminés à partir de consensus d'experts fondés sur la méthode Delphi (méthode qualitative de détermination d'un référentiel) à deux tours [65,68-71]. 
Cette liste a été réactualisée et adaptée à la pratique médicale française par Laroche et al. en 2007 et en 2011 pour être utilisée chez des malades de 75 ans et plus, avec un intérêt supplémentaire : proposer les alternatives pharmacologiques existantes [72,73].

Enfin, en 2015 la liste européenne des médicaments potentiellement inappropriés EU(7)- PIM a compilé et mis à jour ces travaux [74]. Il existe également des recommandations sur les médicaments anticholinergiques grâce à l'ADS (Anticholinergic Drugs Scale).

Les méthodes dites implicites s'appuient sur les données des listes en prenant en compte les comorbidités du patient et son environnement. Elles permettent un jugement clinique au cas par cas. Elles sont ainsi plus spécifiques mais plus chronophages. La méthode implicite DICTIAS pour Diagnostic, Indication, Contre-indication, Tolérance, Interaction, Ajustement de dose, Sécurité, a été développée par le collège des gériatres et a prouvé son intérêt dans de nombreuses études [67].

En 2008, l'outil STOPP/START (Screening Tool of Older Persons' Prescriptions/Screening Tool to Alert to Right Treatment) a été élaboré en Irlande en utilisant la méthode de consensus Delphi. Il correspond à une liste de critères explicites, validée par des experts européens (médecins généralistes, gériatres, neuropsychiatres, pharmaciens), qui permet l'évaluation des traitements médicamenteux prescrits aux patients de 65 ans et plus. Cet outil s'intègre à une démarche globale d'amélioration de la PEC du patient âgé [75]. Chaque critère est accompagné d'une description concise et explicative sur la prescription potentiellement inappropriée [31].

Cet outil permet de cibler les médicaments les plus couramment prescrits en gériatrie et de détecter [76,77] :

- Les principales interactions médicament-médicament et médicament-comorbidité, les effets indésirables en lien avec la prescription de ces médicaments (critères STOPP);

- L'omission de traitements considérés comme appropriés (critères START), en l'absence de contre-indication et selon les systèmes physiologiques $[78,79]$.

La liste START and STOPP a été mise à jour en 2015 et traduite en langue française. Elle se compose maintenant de 115 critères : passage de 22 à 34 critères en 9 sections qui ciblent 30 situations cliniques pour la liste START et de 65 à 81 critères en 12 sections qui ciblent 42 substances/classes médicamenteuses pour la liste STOPP [80,81]. 


\section{iii. Adaptation posologique}

Comme vu précédemment, le vieillissement peut avoir des conséquences sur l'action des médicaments en considérant notamment les paramètres pharmacocinétiques: effets sur l'absorption, la distribution, le métabolisme et l'excrétion des médicaments.

La diminution de la filtration glomérulaire du sujet âgé justifie d'utiliser, pour les médicaments à élimination rénale ou dont le métabolite actif est éliminé par le rein, des posologies adaptées à la fonction rénale [82].

Celle-ci doit être évaluée en pratique à partir de la créatininémie, par l'estimation du DFG [83] et non plus par l'estimation de la clairance de la créatinine selon la formule de Cockcroft et Gault.

En effet, la fiabilité des méthodes utilisées et celle des techniques de dosage de la créatinine ont été réévaluées par la HAS en 2012. L'équation CKD-EPI pour estimer le DFG et la méthode enzymatique standardisée IDMS (Isotopic Dilution Mass Spectroscopy) pour doser la créatinine, qui sont les procédés les plus performants, sont désormais à utiliser.

Cependant, chez les sujets de plus de 75 ans, l'équation CKD-EPI n'est pas encore totalement validée. Les résultats sont à interpréter avec précaution, en les confrontant avec la clinique et avec le résultat des autres examens. Par ailleurs, l'adaptation des posologies des médicaments se fait actuellement en fonction de la clairance estimée par la formule de Crockcroft et Gault, comme indiqué dans les résumés des caractéristiques des produits (RCP). Une révision de ces RCP permettant d'adapter les posologies selon le DFG estimé par l'équation CKD-EPI est désormais souhaitable.

Il existe des sites permettant d'obtenir des recommandations sur une éventuelle adaptation posologique en fonction du DFG des patients, notamment le site GPR (Guide de Prescription et Rein) [84]. Il permet d'obtenir un schéma d'administration du médicament adapté à la fonction rénale du patient (diminution de posologie, durée définie, suspension du traitement si contre-indication de la molécule en cas d'IR modérée ou sévère). 


\section{iv. Observance au traitement}

L'observance thérapeutique se définit comme la capacité à prendre correctement son traitement, c'est-à-dire tel qu'il est prescrit par le médecin. Elle comprend l'administration médicamenteuse : posologie, plan de prise, fréquence de prises, ainsi que l'application des règles hygiéno-diététiques, le suivi médical et les visites de contrôle [85].

Les principaux facteurs de risque de mauvaise observance sont représentés par la polymédication, la complexité des schémas posologiques et le manque de communication entre le patient et ses professionnels de santé [86].

Le défaut d'observance aux traitements peut être associé à :

- Une évolution défavorable de la maladie,

- Une dégradation de la qualité de vie,

- Une augmentation des risques d'hospitalisation,

- Une augmentation des coûts de santé $[87,88]$.

Concernant à l'extrême la non-observance, elle peut être [88] :

- Liée au patient. : due à un défaut de connaissance ou de motivation, des croyances ou à l'absence de relation de confiance avec le médecin.

- Liée au contexte socio-économique, psychologique ainsi qu'au mode de vie du patient.

- Liée au traitement lui-même, comme la complexité du traitement, le coût, la durée, le nombre de prises multiples.

- Liée au soignant de par l'information délivrée et la relation médecin-malade (écoute, empathie).

De manière générale, il est démontré que la non-observance au traitement prescrit est plus fréquente si :

- La pathologie est chronique,

- Les patients sont d'un âge avancé,

- Les patients sont polymédiqués,

- Le nombre quotidien de prises est plus élevé,

- La durée du traitement est plus longue,

- La symptomatologie est peu ou pas symptomatique [88]. 
Enfin, l'observance réside en une excellente relation médecin-malade et en la délivrance d'explications claires et appropriées par le corps médical [87]. Dans ce contexte et devant la complexité de ces paramètres pharmacologiques, le rôle du pharmacien clinicien est essentiel pour optimiser la PECM du patient âgé, toujours en lien avec les équipes médicales.

\section{c) Rôle du pharmacien clinicien}

\section{i. Contexte réglementaire}

L’Ordonnance n²016-1729 du 15 décembre 2016 relative aux pharmacies à usage intérieur (PUI) a confié aux pharmaciens la mission de mener toute action de pharmacie clinique en vue de contribuer à la sécurisation, à la qualité, à la pertinence et à l'efficience des soins, en collaboration avec les autres membres de l'équipe de soins et en y associant le patient [89].

Les travaux de la Direction Générale de l'Offre de Soins (DGOS) mesurent le déploiement des pratiques de pharmacie clinique, que sont :

- Prévenir et corriger les EM potentiellement iatrogènes (Enquête Nationale sur les Evénements Indésirables liés aux soins ENEIS)

- Transmettre des informations complètes et exactes sur le traitement du patient aux points de transition de son parcours de soins.

Ainsi, le Guide « Conciliation des traitements médicamenteux en établissement de santé » a été publié par la HAS en décembre 2016 et mis à jour en février 2018 [6].

Plus récemment, le nouveau décret PUI du 21 mai 2019 (n²019-489) décrit les actions de pharmacie clinique dont la réalisation du bilan de médication (défini à l'article R. 5125-33-5) et de la CTM qui correspond au socle de ce bilan.

Les actions de pharmacie clinique décrites dans ce dernier décret sont les suivantes [7] :

- L'expertise pharmaceutique clinique des prescriptions faisant intervenir des médicaments, produits ou objets mentionnés à l'article L. 4211-1 ainsi que des dispositifs médicaux stériles aux fins d'assurer le suivi thérapeutique des patients ;

- La réalisation de bilans de médication définis à l'article R. 5125-33-5 ;

- L'élaboration de plans pharmaceutiques personnalisés (PPP) en collaboration avec les autres membres de l'équipe de soins, le patient, et, le cas échéant, son entourage ; 
- Les entretiens pharmaceutiques et les autres actions d'éducation thérapeutique auprès des patients ;

- L'élaboration de la stratégie thérapeutique permettant d'assurer la pertinence et l'efficience des prescriptions et d'améliorer l'administration des médicaments.

Les missions de pharmacie clinique deviennent donc prédominantes dans le métier du pharmacien hospitalier et sont mises en pratiques grâce à différentes activités journalières que nous allons détailler à présent.

\section{ii. Analyse pharmaceutique}

L'analyse pharmaceutique de l'ordonnance fait partie intégrante de l'acte de dispensation et permet la vérification des posologies, des doses, des durées de traitement, du mode et des rythmes d'administration, de l'absence de contre-indications, d'interactions et de redondances médicamenteuses.

Elle est à distinguer de la « validation pharmaceutique » qui correspond à une action interne à une pharmacie concluant une analyse pharmaceutique, autorisant la délivrance de produits de santé [90]. Cette activité est quantifiée chaque année dans le cadre du CAQES au niveau régional Provence-Alpes Côte d'Azur (PACA) par l'indicateur obligatoire Q19 : « taux de prescriptions validées par le pharmacien avant délivrance » [91].

\section{iii. Entretien pharmaceutique}

Un entretien pharmaceutique correspond à un échange entre un patient et un pharmacien et permet de recueillir, de rassembler des informations et de renforcer les messages de conseil, de prévention et d'éducation.

Il est abordé selon des objectifs prédéfinis et adaptés au patient et peut être réalisé dans le cadre [90] :

- D'une CTM (entrée ou sortie),

- D'une évaluation et/ou d'un renforcement de l'adhésion thérapeutique,

- D’une action éducative ciblée. 
Il peut être réalisé lors d'une hospitalisation conventionnelle, d'une hospitalisation de jour (HDJ), d'une consultation externe, de manière conventionnée à l'officine ou dans le cadre d'une maison de santé et peut être conduit dans le contexte d'une consultation pluridisciplinaire. Un compte rendu de cet entretien doit être intégré au dossier patient [90].

\section{iv. Bilan de médication}

Un bilan de médication est un entretien structuré avec un patient, dont l'objectif est d'améliorer le bon usage des médicaments. Il permet d'optimiser les prescriptions médicamenteuses et d'éviter l'overuse, le misuse ou l'underuse que nous avons vu précédemment [90].

Le décret $n^{\circ}$ 2011-375 du 5 avril 2011 [92] mentionne « qu'un bilan de médication comprend l'évaluation de l'observance et de la tolérance du traitement ainsi que tous les éléments prévus avec le médecin pour le suivi du patient. Dans ce bilan, le pharmacien recense les effets indésirables et identifie les interactions avec d'autres traitements en cours dont il a connaissance. Il s'assure du bon déroulement des prestations associées ».

L'arrêté du 28 novembre 2016 paru au Journal Officiel de la République Française (JORF) $\mathrm{n}^{\circ} 0279 \mathrm{du}$ 01/12/2016 [93] confirme que le pharmacien peut réaliser un bilan de médication et recueillir dans le dossier médical personnel, auprès du patient ou du prescripteur, dans l'objectif de détecter d'éventuelles contre-indications par rapport aux résultats d'analyses biologiques, aux antécédents, au diagnostic établi. Le pharmacien peut également évaluer le choix d'une molécule et proposer une alternative thérapeutique mieux adaptée au prescripteur afin d'établir une nouvelle ordonnance si nécessaire.

La rédaction d'une intervention pharmaceutique (IP) est conseillée lorsque le pharmacien identifie un problème mettant en jeu l'efficacité ou la sécurité du traitement. Elle permet la formalisation écrite de l'analyse pharmaceutique et sa transmission éventuelle au prescripteur [93].

Dans le nouveau décret $n^{\circ}$ 2019-489 du 21 mai 2019 évoqué précédemment [7], le bilan de médication correspond à la conciliation médicamenteuse à l'entrée et à l'expertise pharmaceutique clinique au sein d'une équipe médicale spécialisée.

Il se déroule sous la forme d'un entretien structuré avec le patient ayant pour objet de recueillir les informations sur l'observance, la perception des traitements, les modalités de prise et les 
effets indésirables. De façon pluridisciplinaire, il permet donc d'identifier les patients ou situations à risque nécessitant un PPP.

\section{v. Plan pharmaceutique personnalisé}

Le PPP réalisé par le pharmacien peut être un entretien pharmaceutique ciblé (adhésion thérapeutique, éducation thérapeutique...) ou une expertise pharmaceutique clinique (conseils de bon usage des produits de santé, plan de prise construit avec le patient).

Il permet donc d'établir un projet formalisé de suivi thérapeutique individualisé dans un souci de continuité des soins [90] dans le cadre du lien ville-hôpital.

\section{vi. Éducation thérapeutique du patient}

Comme nous venons de l'aborder, un PPP peut être associé à l'éducation thérapeutique du patient (ETP). L'ETP nécessite d'adopter une posture d'écoute active.

L'ETP correspond à $[94,95]$ :

- Des programmes autorisés et financé par l'ARS,

- Un ensemble de professionnels de santé sous la responsabilité d'un coordinateur,

- Un bilan éducatif avec des séances (mise en œuvre de moyens adaptés aux objectifs négociés) et une synthèse (évaluation de l'atteinte de ces objectifs),

- Une intégration au parcours de soins (recommandations de la HAS),

- Un accord du patient nécessaire,

- Un ensemble coordonné d'activités d'éducation,

- Une logique pluriprofessionnelle intégrée dans un projet thérapeutique global,

- Des séances animées multidisciplinaires par des professionnels de santé,

- Des objectifs : rendre le patient autonome (compétences d'autosoins et compétences d'adaptation), faciliter son adhésion thérapeutique et améliorer sa qualité de vie. 
vii. Conciliation des traitements médicamenteux

Les personnes âgées à risque de iatrogénie sont aussi plus souvent hospitalisées, et l'hospitalisation peut conduire à une discontinuité de leur traitement [96]. Dès lors, les modifications des prescriptions pendant et après une hospitalisation spécialisée représentent un enjeu majeur. Ainsi, l'HAS recommande de mettre en place l'activité de CTM dans le guide « Conciliation des traitements médicamenteux en établissement de santé », publié en décembre 2016 et mis à jour en février 2018 [6].

Nous allons donc à présent détailler les modalités de mises en place et de suivi de cette activité en établissement de santé (ES).

\section{3) CONCILIATION DES TRAITEMENTS MEDICAMENTEUX}

a) Définition de la conciliation des traitements médicamenteux

La CTM est définie comme un processus formalisé prenant en compte tous les médicaments pris et à prendre par le patient lors de l'élaboration d'une prescription à l'hôpital. Elle associe le patient et repose sur le partage d'informations et sur une coordination interactive et pluriprofessionnelle entre les différents acteurs de soins (médecins, infirmiers, pharmaciens) [97]. L'enjeu principal est de prévenir ou corriger les EM potentiellement iatrogènes, en favorisant la transmission d'informations complètes et exactes sur les médicaments du patient aux points de transition que sont l'admission, la sortie et les transferts du patient [6].

\section{i. $\quad$ Conciliation des traitements médicamenteux d'entrée}

La CTM d'entrée est un acte pharmaceutique qui consiste à établir une liste fiable et exhaustive de tous les médicaments pris par le patient, prescrits ou non (automédication). Elle permet ainsi d'optimiser la prescription lors de l'entrée du patient et la continuité des soins entre la ville et l'hôpital [89]. Elle sécurise la prescription hospitalière et représente un document utile pour les médecins hospitaliers. Elle est issue du bilan médicamenteux optimisé (BMO). 
Le BMO correspond à une démarche structurée qui permet d'établir la liste exhaustive et complète des médicaments pris ou à prendre par le patient avant son hospitalisation (soit prescrits par le médecin traitant ou spécialiste, soit pris en automédication).

La fiche de conciliation des traitements (FCT) est un document qui permet la comparaison entre le bilan médicamenteux et l'ordonnance en cours et précise pour chaque ligne de médicament :

- Le statut du médicament (poursuivi, arrêté, modifié, suspendu, substitué)

- L'existence d'une divergence

- Le caractère intentionnel (divergence intentionnelle (DI)) ou non de la divergence (divergence non intentionnelle (DNI))

- La décision médicale relative aux divergences signalées

- Les sources d'informations utilisées pour établir le BMO.

\section{ii. Conciliation des traitements médicamenteux de sortie}

La CTM de sortie est un processus interactif qui garantit la continuité du traitement médicamenteux en favorisant le lien ville-hôpital. Elle permet la transmission d'une information juste et validée relative au traitement global du patient vers :

- Les professionnels de santé d'aval :

- Médecin traitant, en intégrant à sa nouvelle prescription les modifications apportées au traitement pendant l'hospitalisation,

- Pharmacien d'officine, en optimisant son acte de dispensation,

$\circ \mathrm{ES}$,

○ Établissement d'hébergement pour personnes âgées dépendantes (EHPAD).

- Le patient, si ses capacités cognitives et son état de conscience le permettent afin de l'aider à poursuivre son traitement médicamenteux.

Ce document compare le traitement habituel du patient soit le BMO et de sortie du patient en mentionnant les modifications apportées pendant l'hospitalisation (ajout, arrêt, modification de posologie, ...) et apporte si besoin des compléments d'informations sur le bon usage du médicament. 


\section{b) Contexte réglementaire}

La HAS a publié un guide de mise en œuvre de la conciliation médicamenteuse dans les ES en décembre 2016 et mis à jour en 2018 [6]. Depuis, la CTM fait partie des missions des PUI (Ordonnance du 15 décembre 2016, décret $n^{\circ}$ 2019-489 du 21 mai 2019) comme activité de pharmacie clinique [89].

Cette activité apparait également dans l'obligation du CAQES « Mise en œuvre d'une stratégie de déploiement de la pharmacie clinique intégrée à la politique de management de la PECM et de la conciliation médicamenteuse » avec l'indicateur national Q7 sur le « Déploiement de la conciliation médicamenteuse chez les patients priorisés sur la base d'une analyse des risques » [91].

Pour chaque ES, cet indicateur doit être transmis chaque année sous la forme du « Nombre de patients priorisés et bénéficiant d'une conciliation médicamenteuse d'entrée et/ou de sortie / Nombre de patients priorisés hospitalisés » [98].

c) Objectifs de la conciliation des traitements médicamenteux

Selon la HAS, les problèmes liés à la thérapeutique médicamenteuse sont à l'origine de $21,7 \%$ des hospitalisations des personnes âgées [6].

La CTM vise donc à :

- Sécuriser la PECM du patient tout au long de son parcours en interceptant les EM et en favorisant le lien ville-hôpital

- Améliorer l'observance du patient et la connaissance de ses traitements

- Identifier les causes iatrogènes à l'hospitalisation et donc contribuer à la maîtrise des coûts de PEC des EIM. 


\section{d) Types de divergences rencontrées}

Lors de la CTM, que cela soit à l'entrée ou à la sortie du patient, il est possible de rencontrer des divergences entre les traitements.

Comme nous l'avons vu précédemment, celles-ci peuvent être intentionnelles (documentées et décidées par les équipes médicales selon l'état du patient) ou non intentionnelles (non documentées) aussi qualifiées d'EM.

Les différents types de DNI rencontrées peuvent être des :

- Omissions (oubli d'un ou plusieurs médicaments)

- Erreurs de dosage

- Erreurs de posologie

- Erreur de plan de prise

- Erreur de forme galénique

- Erreur de voie d'administration

- Erreur de principe actif (PA) (médicament prescrit différent).

\section{e) Critères de priorisation}

Afin d'aider les ES à prioriser leurs activités de pharmacie clinique compte-tenu de leur caractère chronophage, des critères de priorisation à la réalisation de la CTM pour les patients hospitalisés ont été proposés par plusieurs OMEDIT [8-10] tels que :

- Âges extrêmes $(<15$ ans et $\geq 75$ ans [9], $\geq 65$ ans [8,10]) : risque iatrogénique lié à âge (arrêté RETEX du 6 avril 2011, patients à risque)

- Certaines situations cliniques à risque :

○ Grossesse [10]

- Insuffisants rénaux (patients en IR sévère) [8-10]: risque iatrogénique lié à l'IR, augmentation du temps de demi-vie des médicaments, accumulation du médicament ou de ses métabolites dans l'organisme.

○ Patients cirrhotiques [9,10]: risque iatrogénique lié à l'insuffisance hépatique, insuffisance hépato-cellulaire (IHC), hypertension portale pouvant aboutir à la 
formation de varices œsophagiennes à l'origine d'hémorragies digestives, état précancéreux.

- Comorbidités $[8,9]$ :

- Patients diabétiques : risques iatrogéniques liés à l'insuline (Never events) et aux antidiabétiques oraux (ISMP's List of High-Alert Medications)

- Patients atteints d'un cancer: risque iatrogénique lié au patient et à la chimiothérapie, toxicité importante des chimiothérapies anticancéreuses, médicament à marge thérapeutique étroite, complexité des schémas thérapeutiques, polymédication importante (chimiothérapies, soins de support, traitement des comorbidités associées), risque élevé d'interactions médicamenteuses, susceptibilité particulière aux EIM des patients atteints de cancer.

- Présence de plus de 3 comorbidités ou score de Charlson $\geq 2[10,99]$.

- Polymédication ( $\geq 5$ médicaments prescrits) [8-10]

- Médicaments à risque prescrits [8-10] : risque iatrogénique lié à certains médicaments (arrêté du 6 avril 2011 (RETEX), concernant notamment les médicaments à marge thérapeutique étroite) :

- Anticoagulants oraux ou injectables (Never events)

- Deux médicaments au moins pour le traitement des pathologies cardiovasculaires (sauf anticoagulants)

○ Digoxine (marge thérapeutique étroite)

○ Immunosuppresseurs (marge thérapeutique étroite)

- Médicaments pour le traitement de l'épilepsie

- Autres (au choix de l'ES).

- Provenance du patient, facteurs relatifs aux organisations à risque (entrée non programmée, service d'urgences) [10]

Ces outils ont pour but d'identifier les services et patients à risque pour leur donner la priorité mais pas l'exclusivité [9]. Chaque établissement peut donc choisir ses propres critères de priorisation des patients selon leur spécificité de services. 


\section{4) PRESENTATION DE L'ETUDE}

Nous allons désormais présenter le contexte et l'objectif de notre étude, pour ensuite décrire certains aspects complémentaires de la méthodologie qui ne figureront pas dans l'article et les différents outils utilisés.

\section{a) $\underline{\text { Contexte }}$}

Le Centre Gérontologique Départemental (CGD) de Marseille est un établissement sanitaire et médico-social spécialisé dans la PEC de la personne âgée. Il a une capacité de plus de 500 lits et places dont :

- 81 lits en médecine chirurgie obstétrique (MCO)

- 45 lits en hospitalisation à domicile (HAD)

- 53 lits en SSR

- 150 lits en USLD

- 218 lits en EHPAD.

La dispensation nominative est réalisée de façon automatisée pour l'ensemble des services de l'établissement. Les activités de pharmacie clinique ont été mises en place dans les unités de soins en lien avec les équipes médicales.

La conciliation médicamenteuse a notamment été mise en place et a été déployée en 2019 dans les services de courts et moyens séjours uniquement. En effet, le Court séjour gériatrique (CSG), le Court séjour Alzheimer (CSA), l'Unité cognitivo-comportementale (UCC) et le service de Soins de suites et de réadaptation (SSR) sont des services cibles prioritaires de la conciliation médicamenteuse car des modifications de traitements y sont très fréquentes et ce sur une courte période (par rapport à d'autres services de longs séjours).

Cette activité a été mise en place sur critères de priorisation définis en commission du médicament et des dispositifs médicaux stériles (COMEDIMS) en lien avec les équipes médicales de l'établissement : admission d'un service hospitalier, insuffisance rénale sévère (IRS, clairance de la créatinine selon Cockcroft-Gault $<30 \mathrm{~mL} / \mathrm{min}$ ) et prescription de médicaments à risque. 


\section{b) Objectifs}

L'objectif principal de notre étude était d'analyser des facteurs prédictifs de risque de DNI sur la prescription d'entrée du patient âgé hospitalisé dans le cadre de la conciliation médicamenteuse.

L'objectif secondaire était donc de définir des critères de priorisation des patients pertinents et adaptés à la population de notre établissement.

c) Méthodes

\section{i. Conciliation des traitements médicamenteux}

Avant d'entamer toute démarche de conciliation médicamenteuse, l'accord des équipes médicales en charge du patient devait être recueilli (par mail ou téléphone).

- Conciliation des traitements médicamenteux d'entrée :

Le lendemain de l'entrée du patient priorisé, l'interne en pharmacie (ou le pharmacien en son absence) se rendait dans l'unité de soins afin de recueillir les informations sur les médicaments du patient afin de réaliser la conciliation médicamenteuse d'entrée.

\section{○ Sources d'informations :}

Plusieurs sources d'informations ont pu être consultées :

$\checkmark$ Dossier médical du patient (papier et logiciel hospitalier Osiris ${ }^{\circledR}$ )

$\checkmark$ Courriers médicaux (médecin traitant, urgences, spécialistes)

$\checkmark$ Entretien avec le patient ou son entourage, si possible (selon avis médical)

$\checkmark$ Appel de la pharmacie d'officine avec recueil des ordonnances des trois derniers mois

$\checkmark$ Médicaments du domicile et/ou prescriptions apportés par le patient.

Le nombre de sources d'informations à consulter devait être au minimum de 3.

L'entretien avec le patient (ou son entourage) était réalisé selon une trame semi-structurée (Complément électronique 1) pour évaluer l'observance du patient et la connaissance de ses traitements. 
Cette recherche permettait d'établir la liste exhaustive de tous les médicaments du patient qu'ils soient prescrits ou non (automédication, compléments alimentaires, phytothérapie, homéopathie, usage cutané, ...).

○ Fiche de conciliation des traitements :

L'interne en pharmacie rédigeait une FCT à l'entrée (Complément électronique 2) qui était validée par le pharmacien et le médecin référent du patient. Cette étape était indispensable pour garantir la qualité du document et lui permettre d'être utilisé tout au long du parcours de soins du patient.

Cette fiche renseignait :

$\checkmark$ Les informations générales du patient (nom, prénom, date de naissance, service)

$\checkmark$ Les coordonnées du médecin traitant, pharmacien d'officine, \pm EHPAD, \pm IDE

$\checkmark$ La date de la conciliation médicamenteuse, nom du pharmacien

$\checkmark$ Les sources d'informations consultées

$\checkmark$ Le BMO :

○ Liste et statut des médicaments établis suite à la recherche (poursuivi, arrêté, modifié, suspendu, substitué, ajouté)

- Ordonnance prescrite à l'entrée

○ Évaluation des divergences entre les deux listes (intentionnelles ou non)

○ Gestion de ces divergences / commentaires.

En cas de DNI, l'interne en pharmacie contactait le médecin prescripteur pour l'informer et mettre à jour si nécessaire la prescription selon les informations recueillies (via téléphone, mail ou contact direct dans le service).

- Traçabilité de la conciliation des traitements médicamenteux d'entrée :

Enfin, le bilan médicamenteux et tous les documents récupérés pour la conciliation médicamenteuse (notamment les ordonnances de l'officine) étaient archivés dans le dossier patient informatisé (DPI) pour être réutilisable lors de la conciliation de transfert ou de sortie du patient hospitalisé. 
- Conciliation médicamenteuse de sortie :

- Bilan médicamenteux de sortie:

En pratique, lorsque la date de sortie du patient était définie, les équipes médicales ou les assistants médico administratifs prévenaient l'interne en pharmacie (ou le pharmacien en son absence) pour qu'il puisse formaliser le bilan médicamenteux de sortie (BMS) du patient (Complément électronique 3).

Ce document comparait le BMO et la prescription de sortie du patient en mentionnant les modifications apportées pendant l'hospitalisation (ajout, arrêt, modification de posologie, ...) et apportait si besoin des compléments d'informations sur le bon usage du médicament.

- Entretien pharmaceutique de sortie :

Un entretien pharmaceutique de sortie était réalisé avec les patients sortants afin de faire le point sur les thérapeutiques modifiées et conservées quand cela était possible.

- Traçabilité de la conciliation médicamenteuse de sortie :

Après validation du médecin référent et du pharmacien, ce bilan était intégré à la «lettre de liaison hospitalisation » adressée au médecin traitant et accompagné si besoin d'une lettre explicative (Complément électronique 4).

Dans le cas d'un retour à domicile, le bilan était également adressé à la pharmacie d'officine du patient.

\section{ii. $\quad$ Analyse des prescriptions}

En parallèle, l'interne en pharmacie (ou le pharmacien en son absence) réalisait une analyse pharmaceutique de la prescription et renseignait les IP proposées sur la FCT :

$\checkmark$ Substitution de MPI chez la personne âgée avec analyse selon les critères STOPP / START

$\checkmark$ Adaptation de posologies si IR (GPR) ou insuffisance hépatique

$\checkmark$ Analyse des interactions médicamenteuses (y compris avec la phytothérapie)

$\checkmark$ Adaptation des formes galéniques si troubles de la déglutition

$\checkmark$ Proposition d'éducation thérapeutique (antivitamine-K (AVK), anticoagulants oraux). 


\section{iii. Recueil des données}

Le recueil et l'analyse des données de CTM effectuées à l'entrée et à la sortie des patients ont été réalisés grâce à un tableau Excel $^{\circledR}$ sur une période de 10 mois (de juin 2019 à mars 2020) de façon prospective.

Les données sur la CTM d'entrée concernaient :

- L'identité et l'âge du patient

- Le service d'hospitalisation

- La date d'admission dans l'ES

- La provenance du patient (domicile, urgences, service hospitalier)

- Le motif d'hospitalisation

- Les antécédents du patient

- Les paramètres cliniques et biologiques du patient à son entrée dans le service

- Le nombre de lignes de traitement à l'admission

- Le nombre de critères de priorisation ayant permis de choisir le patient pour la CTM

- Le nombre et le type de sources d'informations utilisées

- Le nombre et le type de divergences rencontrées

- Le nombre et le type d'IP transmises et prises en compte par le médecin prescripteur.

Les données sur la CTM de sortie concernaient :

- La date de sortie du patient

- Le nombre de lignes de traitement à la sortie

- Les paramètres biologiques présents sur le bilan le plus proche de la sortie

- Le nombre et les types de divergences rencontrées

- Le nombre et le type d'IP transmises et prises en compte par le médecin prescripteur.

\section{iv. Analyse statistique}

Afin d'une part d'évaluer si les critères de priorisation définis au sein de notre ES étaient pertinents et d'autre part d'étudier si d'autres paramètres seraient à prioriser dans notre population gériatrique à risque, des analyses statistiques ont été effectuées sur notre cohorte de patients conciliés à l'entrée. 
Une analyse descriptive de la cohorte de patients a été réalisée. Le but de cette analyse consiste à décrire des données exhaustives à l'aide de moyens appropriés : valeurs calculées (moyenne, médiane, écart-type, ...) ou représentations graphiques [100]. Les résultats seront exprimés en moyenne pour les variables qualitatives et en pourcentage pour les variables quantitatives.

Les critères de priorisation ont été étudiés à l'aide de tests statistiques permettant des analyses inférentielles. Le but de ces analyses est d'effectuer des estimations et des prévisions à partir d'un sous-ensemble de la population [100].

L'analyse inférentielle simple a été réalisée à l'aide de plusieurs tests :

- Test U de Mann-Whitney : permet de tester l'hypothèse selon laquelle la distribution des données est la même pour deux groupes. Il s'agit d'un test non-paramétrique c'està-dire un test qui ne repose pas sur une hypothèse de distribution des données [101].

- Test $t$ de Student : permet de comparer les moyennes de deux groupes d'échantillons distribués suivant la loi normale afin de savoir si les moyennes des deux groupes sont significativement différentes au point de vue statistique [102].

- Test de Chi2 : permet de tester l'hypothèse d'absence de relation entre deux variables catégorielles pour des échantillons de grande taille.

- Test exact de Fisher: permet de tester l'hypothèse de l'indépendance entre deux variables qualitatives, de tester si les fréquences observées sur 2 échantillons sont identiques. Il est utilisé pour l'analyse des tableaux de contingence et s'applique aux échantillons de petite taille [103].

L'analyse inférentielle multiple, quant à elle a été réalisée à l'aide de :

- La régression logistique binaire ascendante conditionnelle : est basée sur un modèle proche de celui de la régression logistique. Au lieu d'avoir une ligne par individu, on aura une ligne par choix possible (sous forme d'une variable binaire qui constitue la variable dépendante). Ainsi, ce ne sont plus les caractéristiques des individus qui sont modélisées mais celles des différentes alternatives [104].

- L'ajustement de la régression par test d'Hosmer-Lemeshow : est utilisé pour la qualité d'ajustement de la régression logistique modèles. Il est souvent utilisé dans les modèles de prédiction des risques. Il compare les effectifs attendus des événements et non événements aux effectifs observés afin d'évaluer l'ajustement du modèle aux données [105]. 


\section{PARTIE ARTICLE}

ETUDE OBSERVATIONNELLE DE FACTEURS PREDICTIFS D'ERREURS MEDICAMENTEUSES ET DE CRITERES DE PRIORISATION EN CONCILIATION MEDICAMENTEUSE GERIATRIQUE 

Titre long : Etude observationnelle de facteurs prédictifs d'erreurs médicamenteuses et de critères de priorisation en conciliation médicamenteuse gériatrique

Title of article: Observational study of factors predictive of medication errors and of prioritization criteria in geriatric medication reconciliation

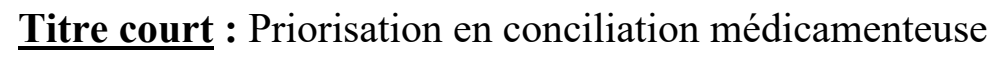

Melissa Abbes ${ }^{1}$, Charlotte Papailhau ${ }^{1}$, Vincent Robert ${ }^{1}$, Dorothée Naudet ${ }^{2}$, Cristel Gille ${ }^{2}$, Caroline Franqui ${ }^{3}$, Catherine Molines ${ }^{4}$, Michel Grino ${ }^{5}$, Marie-Bénédicte Vincentelli ${ }^{1}$

${ }^{1}$ Pharmacie à Usage Intérieur du Centre Gérontologique Départemental de Marseille, 13012, France

${ }^{2}$ Service de Soins et de Réadaptation du Centre Gérontologique Départemental de Marseille, 13012, France

${ }^{3}$ Court Séjour Alzheimer et Unité Cognitivo-Comportementale du Centre Gérontologique Départemental de Marseille, 13012, France

${ }^{4}$ Court Séjour Gériatrique du Centre Gérontologique Départemental de Marseille, 13012, France

${ }^{5}$ Département de Recherche Clinique, Centre Gérontologique Départemental de Marseille, 13012, France

Auteur correspondant

Dr Michel Grino, Département de Recherche Clinique, Centre Gérontologique Départemental, 176 Avenue de Montolivet, 13012 Marseille.michel.grino@gmail.com 


\section{Résumé :}

Objectif : La population gériatrique, atteinte de différentes pathologies et polymédicamentée, est exposée aux risques d'évènements indésirables médicamenteux. La conciliation des traitements médicamenteux (CTM), processus interactif et pluriprofessionnel, garantit la continuité des soins. L'objectif de cette étude était d'analyser et de définir des critères de priorisation pertinents pour la CTM en gériatrie afin d'éviter le maximum d'erreur médicamenteuse (EM).

Méthodes : Un audit clinique de la CTM réalisée aux points de transition que sont l'admission et la sortie du patient a été réalisé de façon prospective pendant 10 mois. Les patients ont été sélectionnés en fonction d'une procédure de priorisation déjà établie dans notre structure qui était la présence d'au moins un des trois critères suivants : provenance d'un service hospitalier, insuffisance rénale sévère et prescription de médicaments à risque.

Résultats : La cohorte de patients conciliés à l'entrée comprenait 136 patients. Les divergences intentionnelles étaient de 8 en moyenne par prescription. Au total, 63 divergences non intentionnelles (DNI) ont été recensées dont la majeure partie (76,2\%) impliquait des omissions de médicament. Trois critères ont été retenus comme facteurs prédictifs indépendants du risque de DNI sur l'ordonnance d'entrée par rapport au bilan médicamenteux optimisé : antécédents rhumatologiques, provenance d'un service hospitalier et hyponatrémie. L'hyponatrémie s'est révélée dans notre étude être le critère qui représentait significativement le plus de risque de présenter une DNI sur l'ordonnance du patient, notamment à risque d'omission de traitement à l'entrée.

Conclusion : Cette étude permettra d'améliorer les critères de priorisation de la procédure de l'établissement de santé ainsi que de mettre en place la CTM en hospitalisation de jour gériatrique afin de renforcer le lien ville-hôpital.

Mots-clés: conciliation des traitements médicamenteux, erreur de médication, service de gériatrie, critères d'admission, étude observationnelle 


\section{Abstract}

Objective: The geriatric population, which suffers from various pathologies and is polymedicated, is exposed to the risk of adverse drug events. Medication reconciliation (MR), which is an interactive and pluriprofessional process, guarantees continuity of care. The objective of this study was to analyze and to define relevant prioritization criteria for MR in older patients in order to avoid a maximum of medication errors.

Methods: A clinical audit of MR at the transition points of patient admission and discharge was conducted prospectively for 10 months. Patients were selected on the basis of a prioritization procedure already established in our structure, that is the presence of at least one of the three following criteria: originating from an hospital department, severe renal failure and prescription of at-risk drugs.

Results: The cohort of reconciled patients at admission included 136 patients. Intentional discrepancies averaged 8 per prescription. A total of 63 unintentional discrepancies (UDs) were identified, the majority of which (76.2\%) involved drug omissions. Three criteria were retained as independent predictors of UDs risk on the entry prescription compared to the optimized drug assessment: rheumatological history, originating from an hospital department and hyponatremia. Hyponatremia was found in the present study to be the most relevant criterion that significantly increased the risk of having an UD on the patient's prescription, particularly a risk of treatment omission at admission.

Conclusion: This study will allow to improve the prioritization criteria on the healthcare establishment's procedure and to implement MR in geriatric day hospitalization in order to strengthen the city-hospital link.

Keywords: medication reconciliation, medication errors, geriatric service, admission criteria, observational study 
AMM : $\quad$ Autorisation de Mise sur le Marché

AVK : $\quad$ Antivitamine $\mathrm{K}$

BMO : $\quad$ Bilan Médicamenteux Optimisé

CAQES : Contrat d'Amélioration de la Qualité et l'Efficience des Soins

CSA : $\quad$ Court Séjour Alzheimer

CSG : $\quad$ Court Séjour Gériatrique

CTM : $\quad$ Conciliation des Traitements Médicamenteux

DFG : $\quad$ Débit de Filtration Glomérulaire

DI : $\quad$ Divergence Intentionnelle

DNI : $\quad$ Divergence Non Intentionnelle

DP : $\quad$ Dossier Pharmaceutique

DPI : $\quad$ Dossier Patient Informatisé

EHPAD : Etablissement d'Hébergement pour Personnes Agées Dépendantes

EIM : $\quad$ Evènement Indésirable Médicamenteux

EM : $\quad$ Erreur médicamenteuse

ES : $\quad$ Etablissement de Santé

ET : $\quad$ Ecart Type

FCT : $\quad$ Fiche de Conciliation des Traitements

GPR : $\quad$ Guide de Prescription et Rein

HAS : $\quad$ Haute Autorité de Santé

IDE : Infirmier Diplômé d'Etat

IMC : $\quad$ Indice de Masse Corporelle

IP : $\quad$ Interventions Pharmaceutiques

IPP : $\quad$ Inhibiteurs de la Pompe à Protons

IR : $\quad$ Insuffisance Rénale

IRS : $\quad$ Insuffisance Rénale Sévère

ISRS : $\quad$ Inhibiteurs Sélectifs de la Recapture de la Sérotonine

MPI : $\quad$ Médicaments Potentiellement Inappropriés

OMA : Ordonnance Médicamenteuse à l'Admission

OMEDIT : Observatoire des Médicaments, Dispositifs médicaux et Innovations Thérapeutiques

PUI : $\quad$ Pharmacie à Usage Intérieur

SIADH : $\quad$ Syndrome de sécrétion Inappropriée d'Hormone Anti-Diurétique

SSR : $\quad$ Soins de Suite et de Réadaptation

UCC : Unité Cognitivo-Comportementale

USLD : $\quad$ Unité de Soins de Longue Durée 


\section{$\underline{\text { INTRODUCTION }}$}

En 2020, le vieillissement de la population française se poursuit, plus d'une personne sur cinq en France étant âgé de 65 ans ou plus [1]. Les principales conséquences pharmacocinétiques et pharmacodynamiques liées à l'âge augmentent la fragilité de ces patients, la coexistence de polypathologies et d'une polymédication et de ce fait le risque d'évènements indésirables médicamenteux (EIM) [2]. L'évaluation du rapport bénéfice/risque et l'optimisation de la prescription des médicaments de façon pluridisciplinaire sont donc essentielles pour améliorer la qualité de la prescription médicamenteuse dans cette population fragile et à risque $[2,3]$. Ainsi, la conciliation des traitements médicamenteux (CTM) est devenue une priorité de la Haute Autorité de Santé (HAS) en lien avec le nouveau décret des pharmacies à usage intérieur (PUI) n²019-489 de mai 2019 et l'indicateur Q7 du contrat d'amélioration de la qualité et l'efficience des soins (CAQES) [4]. Processus interactif et pluriprofessionnel [5], la CTM permet de prévenir ou de corriger les erreurs médicamenteuses (EM) potentiellement iatrogènes, en favorisant la transmission d'informations complètes et exactes sur les médicaments du patient à son admission, sa sortie et ses transferts [6].

Afin d'optimiser au mieux la CTM, les Observatoires des médicaments, dispositifs médicaux et innovations thérapeutiques (OMEDIT) proposent des critères de priorisation des patients de la population générale tels que l'âge, certaines comorbidités (diabète, cancer, ...) ou la présence de médicaments à risque sur l'ordonnance d'entrée. Cependant, ceux-ci ne sont potentiellement pas adaptés à une population gériatrique.

Nous avons donc entrepris une étude sur la CTM chez le sujet âgé car il n'existe pas de critères de priorisation exclusifs proposés par les instances pour réaliser une CTM dans cette population. Chaque établissement peut choisir ses propres critères de priorisation (liés au patient, à la prescription, au type de service ou à l'organisation de la pharmacie). A titre d'exemple, l'OMEDIT Bretagne a mené une étude sur la priorisation du déploiement de la pharmacie clinique selon une approche globale des risques en gériatrie ; en définissant des critères discriminatoires (passage par les urgences, durée moyenne de séjour supérieure à la moyenne nationale) ou non discriminatoires (exemples : âge $>65$ ans, médicaments à risque, insuffisance rénale sévère (IRS)) [7]. Cependant, cette priorisation n'a pas été mise en œuvre dans notre établissement car elle ne concernait pas l'activité de conciliation médicamenteuse à proprement parler. De ce fait, l'originalité de notre travail réside dans l'analyse et la recherche statistique de critères de priorisation pertinents en gériatrie, en comparant une cohorte de patients conciliés 
à la présence finale d'une divergence non intentionnelle (DNI) sur l'ordonnance médicamenteuse à l'admission (OMA).

\section{MATERIEL ET METHODES}

\section{Type d'étude et services concernés}

Cette étude a reçu un avis favorable du comité d'éthique de l'établissement. Un audit clinique de l'activité de conciliation médicamenteuse réalisée aux points de transition spécifiques que sont l'admission et la sortie du patient a été réalisé de façon prospective pendant une durée de 10 mois, de juin 2019 à mars 2020.

Les services concernés étaient des services de soins de courts séjours et de moyens séjours : Court séjour gériatrique (CSG), Soins de suite et de réadaptation gériatrique (SSR), Court séjour Alzheimer (CSA) et Unité cognitivo-comportementale (UCC). Ces derniers assurent la prise en charge des patients âgés à problématique cognitive et/ou comportementale et présentant des conduites d'errance. Une conciliation médicamenteuse d'entrée a été exceptionnellement réalisée en Unité de soins de longue durée (USLD) à la demande du médecin du service.

Nous avons suivi la procédure spécifique à notre établissement, établie en accord avec les équipes médicales, dans laquelle il est convenu de prioriser les patients à risque d'EM (et donc prioritaires pour la conciliation médicamenteuse) s'ils présentaient au moins un des trois critères suivants : provenance d'un service hospitalier (urgences comprises), IRS à l'admission (clairance de la créatinine selon Cockcrauft-Gault $<30 \mathrm{~mL} / \mathrm{min}$ ), prescription de médicaments à risque définis au sein de l'établissement avec les équipes médicales (anticoagulants oraux ou injectables, bi anti-agrégation plaquettaire, immunosuppresseurs, digoxine, anticancéreux oraux, neuroleptiques, clozapine, antibiotiques, corticoïdes oraux). 


\section{Procédures de CTM}

1) Conciliation des traitements médicamenteux à l'admission

\section{a) Sources d'informations}

Plusieurs sources d'informations sur le traitement habituel et/ou d'une précédente hospitalisation ont pu être consultées telles que le dossier médical du patient, les courriers médicaux, l'entretien avec le patient ou son entourage, l'appel de la pharmacie d'officine avec recueil des ordonnances des trois derniers mois, les médicaments du domicile et/ou prescriptions apportés par le patient. Le nombre de sources d'informations à consulter devait être au minimum de 3 . Cette recherche permettait d'établir la liste exhaustive de tous les médicaments du patient qu'ils soient prescrits ou non (automédication, compléments alimentaires, phytothérapie, homéopathie, usage cutané, ...).

L'entretien avec le patient (ou son entourage) était réalisé selon une trame semi-structurée (Complément électronique 1) pour évaluer l'observance du patient et la connaissance de ses traitements.

\section{b) Formalisation des documents}

Une fiche de conciliation des traitements (FCT) (Complément électronique 2) était utilisée et validée par le pharmacien et le médecin référent du patient.

Cette fiche renseignait :

- Les informations générales du patient (nom, prénom, date de naissance, service)

- Les coordonnées du médecin traitant, pharmacien d'officine, \pm établissement d'hébergement pour personnes âgées dépendantes (EHPAD), \pm infirmier diplômé d'état (IDE)

- La date de la conciliation médicamenteuse

- Les sources d'informations consultées

- Le bilan médicamenteux optimisé (BMO) :

- Liste et statut des médicaments établis suite à la recherche (poursuivi, arrêté, modifié, suspendu, substitué, ajouté) 
○ Ordonnance prescrite à l'entrée

○ Évaluation des divergences entre les deux listes (intentionnelles ou non)

○ Gestion de ces divergences / commentaires.

En cas de DNI, l'interne en pharmacie contactait le médecin prescripteur pour l'informer et mettre à jour si nécessaire la prescription selon les informations recueillies. Les types de DNI pouvaient être des omissions, erreur de dosage, de posologie, de plan de prise, de forme galénique, de principe actif. Les types de divergences intentionnelles (DI) pouvaient être des instaurations et/ou adaptations posologiques volontaires (par le médecin du service) au vu de l'état clinique et biologique du patient.

\section{c) Analyse pharmaceutique}

En parallèle, l'interne en pharmacie (ou le pharmacien en son absence) réalisait une analyse pharmaceutique de la prescription à l'entrée, pendant et à la sortie du patient et renseignait les interventions pharmaceutiques (IP) proposées sur la FCT :

- Substitution de médicaments potentiellement inappropriés (MPI) chez la personne âgée avec analyse selon les critères STOPP / START

- Adaptation de posologies si IR (site Guide de Prescription et Rein GPR) ou insuffisance hépatique

- Analyse des interactions médicamenteuses (y compris avec la phytothérapie)

- Adaptation des formes galéniques si troubles de la déglutition

- Proposition d'éducation thérapeutique (antivitamines K, anticoagulants oraux).

\section{d) Traçabilité de la conciliation médicamenteuse d'entrée}

Le bilan médicamenteux et tous les documents récupérés pour la conciliation médicamenteuse (notamment les ordonnances de l'officine) étaient archivés dans le dossier patient informatisé (DPI) pour être réutilisable lors de la conciliation de transfert ou de sortie du patient hospitalisé. 
2) Conciliation des traitements médicamenteux de sortie

a) Bilan médicamenteux de sortie

Ce document comparait le BMO et l'ordonnance de sortie du patient en mentionnant les modifications apportées pendant l'hospitalisation (ajout, arrêt, modification de posologie, ...) et apportait si besoin des compléments d'informations sur le bon usage du médicament (Complément électronique 3).

b) Entretien pharmaceutique de sortie

Un entretien pharmaceutique de sortie était réalisé avec les patients sortants afin de faire le point sur les thérapeutiques modifiées et conservées quand cela était possible.

c) Traçabilité de la conciliation médicamenteuse de sortie

Après validation du médecin référent et du pharmacien, ce bilan était intégré à la « lettre de liaison hospitalisation » adressée au médecin traitant et accompagné si besoin d'une lettre explicative (Complément électronique 4).

Dans le cas d'un retour à domicile, le bilan était également adressé à la pharmacie d'officine du patient.

\section{3) Recueil des données}

Les données suivantes ont été recueillies :

- à l'entrée : provenance, entourage au domicile, antécédents, données anthropométriques, statut d'autonomie et risque de chute, critères de priorisation, traitements, bilan biologique, caractéristiques de la CTM avec le nombre de lignes de prescription, le type d'interventions pharmaceutiques (IP) renseignées et prises en compte ;

- à la sortie : durée de séjour, nombre et type de divergences rencontrées, bilan biologique, traitements, type d'IP renseignées et prises en compte. 


\section{4) Analyse statistique}

Les résultats sont exprimés par la moyenne \pm l'écart type (ET) pour les variables qualitatives et le nombre (\%) pour les variables quantitatives. L'analyse statistique simple a été réalisée à l'aide de Statistical package for the social sciences (SPSS, V23) en utilisant le test U de MannWhitney ou le test $\mathrm{t}$ de Student ou le test de Chi2 ou le test de Fisher. Les variables associées à une valeur de $\mathrm{p}<0,2$ ont été introduites dans un test de régression logistique binaire ascendante conditionnelle. Une valeur de $\mathrm{p}<0,05$ a été considérée significative. La qualité d'ajustement de la régression a été étudiée par le test d'Hosmer-Lemeshow et une colinéarité des variables prédictives indépendantes a été recherchée.

\section{$\underline{\text { RESULTATS }}$}

1) Analyse descriptive de la cohorte de patients

a) Description de la cohorte totale de patients

Au total, l'interne en pharmacie a réalisé 138 de ces conciliations médicamenteuses : 108 à l'entrée et la sortie, 28 uniquement à l'entrée et 2 uniquement à la sortie du patient.

\section{b) Description de la cohorte de patients conciliés à l'entrée}

La répartition des conciliations médicamenteuses d'entrée par service représentait : 72 patients $(52,9 \%)$ au SSR, 57 patients $(41,9 \%)$ au CSG, 5 patients $(3,7 \%)$ au CSA, 1 patient $(0,7 \%)$ à l'UCC et 1 patient $(0,7 \%)$ à 1 'USLD.

Le Tableau 1 présente l'ensemble des données descriptives de la cohorte de patients conciliés à l'entrée. Toutes les données biologiques ont été extraites de l'analyse standard réalisée à chaque entrée du patient $(\mathrm{J}+1)$.

La cohorte de patients conciliés à l'entrée comprenait donc 136 patients avec un ratio H/F de 0,55 (88 femmes et 48 hommes) et une moyenne d'âge de 85,9 ans ( \pm 5,23 ans). 
Concernant la provenance des patients, $75 \%$ des patients étaient adressés par un service hospitalier (dont 8,8 \% d'un service de chirurgie), 5,1 \% par un EHPAD et 19,9\% venaient directement du domicile. Par ailleurs, 70,5\% des patients bénéficiaient d'un IDE au domicile.

Les antécédents cardio-vasculaires représentaient la majorité des antécédents des patients (86\%) suivis des antécédents psychiatriques dont les démences (47,1\%), endocrinologiques $(42,6 \%)$ et rhumatologiques $(38,2 \%)$.

La moitié des patients (50,4\%) présentaient une dépendance majeure (score ADL, activities of daily living, avec un score $<3$ signifiant une dépendance majeure et un score à 0 signifiant une dépendance totale), 59,6\% des troubles cognitifs et $65,7 \%$ des risques de chute.

La majorité des patients présentaient une hypoalbuminémie $(58,2 \%)$ et une hypovitaminose D (73,8 \%). Concernant le degré d'IR à l'entrée, 27,4\% des patients présentaient une IRS selon la formule de Cockroft et Gault et 12,6 \% l'étaient selon la formule CKD EPI.

Le nombre de lignes de traitement à l'entrée était supérieur à 5 pour $97,1 \%$ des patients (polymédication) et supérieur à 10 pour 59,6\% des patients, avec une moyenne de 10 lignes par patient. Concernant la prescription de médicaments à risque, 75,7 \% des patients avaient au moins un de ces médicaments présent sur son ordonnance d'entrée, notamment des anticoagulants $(55,3 \%)$, des antibiotiques $(36,9 \%)$ et des neuroleptiques $(19,4 \%)$.

\section{2) Conciliation des traitements médicamenteux à l'admission}

\section{a) Sources d'informations utilisées}

Du fait de la spécificité gériatrique des patients et des possibles troubles cognitifs associés, les entretiens ne pouvaient souvent pas être réalisés uniquement avec le patient (57,6\%). L'aide des aidants était souvent sollicitée : famille (28\%), famille et patient (12,8\%), IDE libéral $(1,6 \%)$. Le dossier médical était consulté systématiquement. La source externe la plus pertinente s'avérait être la pharmacie d'officine (91,6\%) qui transmettait par messagerie sécurisée les ordonnances des trois derniers mois du patient. Cela permettait de centraliser les ordonnances des différents spécialistes du patient mais également d'avoir un regard sur l'automédication de celui-ci. La constitution d'une liste exhaustive des traitements du patient, prescrits ou non, était donc possible. 
b) Divergences retrouvées lors des conciliations médicamenteuses d'entrée

En moyenne, 9 divergences (intentionnelles ou non intentionnelles) ont été retrouvées par prescription à l'entrée du patient.

Les DI correspondaient à des modifications volontaires de traitement dont les raisons devaient être renseignées dans le dossier patient (ajout, modification ou interruption de traitement). Celles-ci étaient de 8 en moyenne par prescription, avec une médiane de 8 (entre 1 et 18). La réévaluation du traitement habituel du patient par les équipes médicales hospitalières est donc appliquée et nécessaire.

Notre étude a retrouvé une moyenne de 0,8 DNI par prescription. Celles-ci correspondaient à des modifications de traitement involontaires ou erreurs médicamenteuses (ordonnance non transmise, omission de médicament, erreurs de posologie, de voie d'administration ou d'horaire de prise). Cinquante-sept patients présentaient au moins une DNI. Au total, 63 DNI ont été recensées pendant la durée de notre étude dont la majeure partie impliquait des omissions de médicament $(76,2 \%)$. Les différentes causes de DNI étaient les suivantes : 48 omissions (76,2\%), 10 erreurs de posologie (15,9\%), 4 erreurs de dosage $(6,3 \%), 1$ erreur de médicament $(1,6 \%)$.

c) Difficultés rencontrées lors de la conciliation médicamenteuse d'entrée

La principale difficulté rencontrée relevait du caractère non interrogeable du patient dans $47 \%$ des cas, parmi ceux-ci ont été retrouvés des troubles cognitifs du patient (98\%) ou une barrière de la langue $(2 \%)$.

Par ailleurs, il était parfois difficile d'obtenir les 3 sources d'informations minimales nécessaires à la réalisation de la CTM d'entrée :

- A l'entretien : non connaissance des traitements et/ou faux renseignements du patient sur la pharmacie d'officine habituelle aboutissant à des recherches longues et fastidieuses $(1,5 \%)$

- Pas d'ordonnance de ville à l'arrivée du patient dans le service 
- Patient en rupture de traitement : difficultés de recherche de traitement par appels aux différentes pharmacies d'officine $(2,2 \%)$

- Patient isolé $(1,5 \%)$

- Patient vivant à l'étranger : longues recherches de pharmacie d'officine à l'étranger $(1,5 \%)$

- Pas de réponse de l'établissement de santé (ES) précédant l'hospitalisation (0,7\%)

- Écriture du médecin difficilement déchiffrable : appel de la pharmacie d'officine pour avoir la confirmation du traitement délivré $(2,2 \%)$

- Nomadisme de médecin et de pharmacie d'officine.

3) Interventions pharmaceutiques

Grâce à l'analyse pharmaceutique réalisée en parallèle des conciliations médicamenteuses, plusieurs IP ont pu être transmises aux équipes médicales. Le nombre d'IP totales transmises, leur taux d'acceptation ainsi que leurs détails sont présentés dans le Complément électronique 5.

\section{4) Conciliation des traitements médicamenteux de sortie}

Parmi la cohorte de patients, 108 ont pu bénéficier d'une conciliation médicamenteuse de sortie. Parmi les 28 patients restants, 14 patients ont été transférés dans un autre hôpital (ou service), 4 sont retournés à domicile (mais dans ces deux cas sans que la pharmacie ne soit prévenue) et 10 patients sont décédés. Les conciliations médicamenteuses de sortie n'ont donc pas pu être réalisées dans les temps ou n'étaient pas indiquées.

5) Analyse des critères de priorisation des patients:

a) Analyse inférentielle simple

La présence d'une DNI lors de la CTM d'entrée a été comparée à chaque critère évoqué dans la partie descriptive de la cohorte. Tous les résultats statistiques de cette analyse sont présentés dans le Tableau 2. Les variables associées à une augmentation des risques de DNI étaient : une diminution de l'index de masse corporelle, les antécédents rhumatologiques, une hyponatrémie, 
une hyperkaliémie, des risques de chute, la provenance d'un service hospitalier de médecine ou d'un service de chirurgie. Les variables associées à une diminution des risques de DNI étaient : les antécédents neurologiques ou urinaires, une leucocytose, des troubles cognitifs, la provenance du domicile, la prescription de médicaments à risque.

\section{b) Analyse inférentielle multiple}

Les critères préalablement sélectionnés en analyse simple ont été inclus dans l'analyse inférentielle multiple. L'ajustement de la régression est de très bonne qualité (Test d'HosmerLemeshow : Chi2 $=5,129 \mathrm{P}=0,400)$ et le modèle permet d'expliquer 22,5\% des DNI $\left(\mathrm{R}^{2} \mathrm{de}\right.$ Nagelkerke). Il n'existe pas de multicolinéarité. Les résultats sont présentés dans le Tableau 3. Ainsi, les prédicteurs indépendants d'une DNI sur l'ordonnance du patient étaient l'hyponatrémie, suivie de la provenance d'un service hospitalier et des antécédents rhumatologiques à type de fractures notamment, alors que des antécédents neurologiques étaient prédicteurs indépendants d'une faible incidence de DNI.

\section{c) Analyse détaillée}

L'analyse détaillée consistait à comparer les quatre critères précédemment retenus (antécédents neurologiques, antécédents rhumatologiques, provenance d'un service hospitalier et hyponatrémie) avec le type de DNI retrouvé (omission d'un médicament, erreur de posologie, erreur de dosage, erreur de médicament). Les résultats sont présentés dans le Tableau 4.

L'omission de médicament et les erreurs de posologie étaient les DNI majoritairement retrouvées lors des CTM d'entrée. Les critères hyponatrémie et provenance d'un service hospitalier du patient sont significativement à risque de retrouver une DNI d'omission de traitement à l'entrée. Les antécédents rhumatologiques montrent une tendance à la significativité à risque de retrouver une DNI d'omission de traitement. Les antécédents neurologiques montrent une tendance à la significativité avec une diminution de retrouver une DNI d'omission de traitement. 


\section{$\underline{\text { DISCUSSION }}$}

Cette étude avait pour objectif d'évaluer des facteurs prédictifs de risque de présence de DNI sur l'ordonnance d'entrée du patient hospitalisé dans des services de gériatrie lors de la conciliation médicamenteuse d'entrée [8]. Elle a été réalisée sur une cohorte de 136 patients conciliés à l'entrée de leur hospitalisation dans des services de courts ou moyens séjours.

Lors de cette étude, 136 CTM à l'admission ont été réalisées : 108 à l'entrée et la sortie, 28 uniquement à l'entrée et 2 uniquement à la sortie du patient. Au total, 63 DNI ont été recensées dont la majeure partie impliquait des omissions de médicament (76,2\%), suivies des erreurs de posologie $(15,9 \%)$, de dosage $(6,3 \%)$ et de principe actif $(1,6 \%)$. Dans l'étude de Boissinot et al., l'omission représentait également la principale catégorie de DNI (56\%), suivis d'erreurs de posologie (33\%) et d'ajout (11\%) [9]. Dans l'étude de Saint-Germain et al., l'omission était également la principale erreur (58 \%) et $33 \%$ des DNI concernaient un médicament du système cardiovasculaire [10].

La comparaison entre le BMO et l'OMA met en avant un grand nombre de médicaments prescrits au patient âgé, souvent atteint de multiples comorbidités. Le nombre moyen de divergences est important et presque égal au nombre de médicaments prescrits ( 9 divergences en moyenne pour une moyenne de 10 médicaments prescrits sur le BMO) comme c'était le cas de l'étude de Boissinot et al. sur une cohorte de patients âgés avec 7,5 divergences en moyenne pour 7,7 médicaments prescrits en moyenne sur le BMO [9].

Les DI rencontrées dans notre étude étaient : des arrêts expliqués notamment par des pratiques locales (arrêt systématique de certains médicaments à l'entrée, par exemple anti-inflammatoires non stéroïdiens, réévaluation de la prise en charge de la douleur) ; des substitutions de médicaments non présents au livret thérapeutique de l'établissement par des spécialités contenues dans le livret ; des ajouts, dont une partie due également à des pratiques locales (protocole de bain de bouche: bicarbonate de sodium + Fungizone $^{\circledR}$, protocole d'insuline : Novorapid $^{\circledR}$ ); des modifications de posologie par une réévaluation thérapeutique des équipes médicales hospitalières qui est primordiale chez le sujet âgé (MPI, risque de chute, arrêt des traitements prescrits sur une trop longue durée par exemple les benzodiazépines, réévaluation de la posologie selon le degré d'IR...).

Dans la présente étude, le nombre moyen de DNI retrouvé par prescription est de 0,8 . Il était de 0,6/prescription dans l'étude de Barthod [11]. Le pourcentage observé de DNI que nous avons 
retrouvées $(46,3 \%)$ se situe dans la moyenne par rapport aux résultats d'autres études répertoriant, en moyenne, de $32 \%$ à $54 \%$ de DNI à l'admission [9,12-15].

Trois critères ont été retenus comme facteurs prédictifs indépendants du risque de DNI sur l'ordonnance d'entrée par rapport au BMO : les antécédents rhumatologiques, la provenance d'un service hospitalier et l'hyponatrémie. Sur les 3 critères de priorisation habituellement utilisés dans notre établissement, seul le critère " provenance d'un service hospitalier » paraît pertinent et 2 nouveaux critères, antécédents rhumatologiques et hyponatrémie, ont été validés par notre étude. On peut émettre l'hypothèse que la provenance d'un service hospitalier, critère qui est cohérent avec ceux des OMEDIT, engendre une multiplicité des prescriptions et un manque de transfert d'informations entre les ES.

Dans l'étude de Boursier et al., l'analyse par régression logistique multiple montre, dans une population hospitalisée en rhumatologie, que le nombre de DNI est lié positivement au nombre de médicaments à l'inclusion et au contact avec l'officine du patient comme source documentaire. La présence de divergences de gravité significative ou critique était associée indépendamment avec les antécédents de goutte, de dyslipidémie et d'insuffisance cardiaque. De ce fait, cibler prioritairement la CTM chez les patients avec un de ces antécédents ou un nombre de médicaments à l'admission $\geq 6$ permettrait de réduire l'activité de $27 \%$, tout en détectant $87 \%$ des divergences significatives [16].

Concernant l'hyponatrémie, elle s'est révélée dans notre étude être le critère qui représentait significativement le plus de risque de présenter une DNI sur l'ordonnance du patient, et plus précisément un risque d'omission de traitement à l'entrée. Dans l'étude de Barbier et al., en analyse multiple, l'hospitalisation en services gériatriques pour désordres hydro-électrolytiques (non précisés) et le nombre de lignes de traitement $>9$ sont des facteurs prédictifs significatifs de DNI [8]. Plusieurs études [17-22] ont montré que la forme chronique légère d'hyponatrémie peut provoquer une augmentation du taux de chutes, probablement en raison d'une altération des mécanismes d'attention, de posture et de marche [17]. En effet, l'hyponatrémie provoquerait une altération des fonctions cognitives [18], des troubles de la marche [19] voire des fractures $[20,21]$. Le lien de causalité entre l'hyponatrémie et le risque de retrouver une DNI telle que l'omission d'un traitement que nous avons mis en évidence pourrait être en partie consécutif à un oubli par le patient de mentionner un ou plusieurs de ses médicaments habituels lors de l'entretien. La réévaluation de la prise en charge médicamenteuse (PECM) de ces patients atteints d'hyponatrémie pourrait éviter un nombre considérable d'hospitalisations [19]. 
De plus, l'hyponatrémie peut être retrouvée lors de l'usage de multiples médicaments : les diurétiques thiazidiques (qui peuvent engendrer un syndrome de sécrétion inappropriée d'hormone anti-diurétique (SIADH), les antidépresseurs inhibiteurs sélectifs de la recapture de la sérotonine (ISRS) ou les inhibiteurs de la pompe à protons (IPP)). Ces médicaments étant souvent trop prescrits en ville et peu réévalués, le lien avec l'existence d'une DNI à l'entrée paraît cohérent (absence de suivi thérapeutique). Dans l'étude de Beuscart et al., des patients âgés de 65 ans et plus prenant au moins cinq médicaments par jour, ont été interrogés afin d'évaluer leur gestion des médicaments à domicile. Dans cette cohorte de patients âgés, plus de 700 inspections d'armoires à pharmacie ont identifié des problèmes liés à un mauvais emplacement ou stockage et à la présence fréquente de médicaments inappropriés ou à risque (15\% au regard des comorbidités et des traitements d'un patient) et redondants [23]. Cette identification des difficultés dans la gestion des médicaments à domicile montre l'intérêt de la réalisation d'une conciliation médicamenteuse à l'entrée de ces patients âgés ambulatoires dans le secteur hospitalier.

Notre travail s'avère être une étude complète dotée d'un suivi précis du patient avec notamment l'analyse des IP réalisées tout au long du parcours du patient. Cependant, elle connaît plusieurs limites. Premièrement, la population que nous avons étudiée a été choisie du fait de la procédure établie de l'ES sur la priorisation des patients pour la CTM, ce qui constitue un biais de sélection évident qui aurait pu masquer ou sélectionner artificiellement un ou plusieurs autres critères prédicteurs de DNI. Toutefois, il est important de remarquer que les nouveaux critères prédicteurs de DNI mis en évidence dans notre étude sont indépendants de ceux prédéfinis par l'établissement. Deuxièmement, il y a eu moins de CTM réalisées dans les services de CSA/UCC. En effet, ces services accueillent une population de patients avec des troubles cognitifs sévères qui seraient le plus à risque d'erreurs médicamenteuses (erreur de plan d'administration, de posologie, de principe actif...) mais l'entretien avec ces patients est compliqué, les informations sont difficiles à centraliser et souvent peu fiables. Troisièmement, un BMO nécessite d'identifier l'ensemble des traitements prescrits ou pris en automédication (nom, dosage et posologie exacts). Ce recueil doit être le plus exhaustif possible. La réalisation de la CTM d'entrée impose une prise en charge transversale entre différents professionnels de santé : médecin traitant, médecin gériatre hospitalier, urgentiste, pharmacien d'officine et pharmacien hospitalier [9]. Depuis octobre 2012, les pharmaciens des PUI tout comme ceux d'officine peuvent accéder au dossier pharmaceutique (DP) [24] et connaître l'ensemble des médicaments délivrés (prescrits par le médecin ou conseillés par le pharmacien) au cours des 
quatre derniers mois, grâce à la lecture des données contenues sur la carte vitale du patient (carte d'assurance maladie française). Or, la PUI de notre établissement ne dispose pas encore du système informatique associé au lecteur de carte vitale qui permettrait l'accès au DP. Une autorisation est nécessaire pour réaliser cette activité. Ainsi, l'accès au DP est à optimiser afin de bénéficier d'une source d'information supplémentaire fiable [9]. Nous n'avons malheureusement pas pu réaliser d'étude du suivi des CTM de sortie (prise en compte des modifications thérapeutiques au cours de l'hospitalisation par le médecin traitant, transfert de ces modifications thérapeutiques à la pharmacie d'officine) en raison de difficultés d'accès aux données patients d'un point de vue administratif. Pour finir, une grande partie des patients conciliés étant en provenance d'un service hospitalier, il serait intéressant d'étudier dans une prochaine étude le type de DNI retrouvées selon la spécificité des services d'où proviennent les patients (urgences, unité d'hospitalisation de courte durée, chirurgie, médecine, ...). 


\section{CONCLUSION}

Cette étude prospective réalisée dans des services exclusivement gériatriques s'inscrit dans un processus global de sécurisation de la PECM aux points de transition du parcours du patient, et ce de façon pluridisciplinaire. L'analyse des facteurs prédictifs de DNI a permis d'identifier des critères pertinents pour prioriser les patients à concilier à l'entrée, en lien avec la spécificité gériatrique de notre établissement: antécédents rhumatologiques, provenance d'un service hospitalier et hyponatrémie sur l'analyse biologique d'entrée. Cette priorisation permettra d'optimiser le temps pharmaceutique dédié à cette activité chronophage tout en interceptant le maximum d'EM. Couplée à l'analyse pharmaceutique réalisée pendant l'hospitalisation, la transmission du BMO au médecin traitant et à la pharmacie d'officine permet de renforcer le lien ville-hôpital, véritable enjeu de santé publique à l'heure actuelle. A l'avenir, la mise en place de la CTM en hôpital de jour gériatrique (avec notamment l'utilisation de ces critères de priorisation) pourra être un nouvel enjeu de sécurisation médicamenteuse d'un point de transition émergent et prioritaire qu'est le secteur ambulatoire.

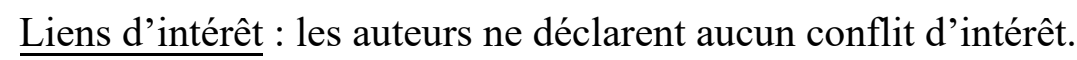

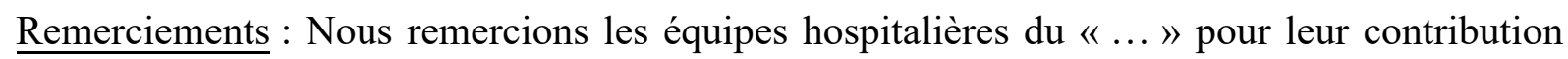
dans ce travail. 


\section{RÉFÉRENCES}

1. INSEE. Bilan démographique. 2019 [Internet]. [cité 11 nov 2020]. Disponible sur : https://www.insee.fr/fr/statistiques/4281618\#titre-bloc-21

2. Legrain S, Lacaille S. Prescription médicamenteuse du sujet âgé. EMC - Médecine. 1 avr 2005;2(2):127-36.

3. Dauphinot V, Jean-Bart E, Krolak-Salmon P, Mouchoux C. A multi-center, randomized, controlled trial to assess the efficacy of optimization of drug prescribing in an elderly population, at 18 months of follow-up, in the evolution of functional autonomy: the OPTIM study protocol. BMC Geriatr. 2017;17(195):1-9.

4. ARS, Omédit PACA-Corse [Internet]. [cité 18 janv 2021]. Disponible sur : https://www.omeditpacacorse.fr/

5. OMEDIT Ile de France. Conciliation médicamenteuse [Internet]. [cité 11 nov 2020]. Disponible sur : http://www.omedit-idf.fr/qualite-securite/parcours-du-patient/concili ation-medicamenteuse-2/

6. Haute Autorité de Santé (HAS), 2018. Mettre en œuvre la conciliation des traitements médicamenteux en établissement de santé [Internet]. [cité 11 nov 2020]. Disponible sur : https://www.has-sante.fr/jcms/c_2736453/fr/mettre-en-oeuvre-la-conciliation-destraitements-medicamenteux-en-etablissement-de-sante

7. Lurton Kolb A. OMEDIT Bretagne, Priorisation du déploiement de la pharmacie clinique selon une approche globale des risques au pole gériatrique rennais [Internet]. [cité 24 janv 2021]. Disponible sur : https://www.omeditbretagne.fr/wp-content/uploads/2020/01/REX -P\%C3\%B4le-G\%C3\%A9riatrique-Rennais-Dr-Agn\%C3\%A8s-KOLB-LURTON.pdf

8. Barbier G, Berthou J, Koeberle S, Amsallem A, Nerich V, Limat S, et al. Sécurisation du parcours patient en gériatrie: quels facteurs de risque d'erreurs médicamenteuses à l'admission? Le Pharmacien Hospitalier et Clinicien. 2020;55(3):275-81.

9. Boissinot L, Bachalat N, Perrier-Cornet E, Leglise P, Huchon-Bécel D. Conciliation des traitements médicamenteux en gériatrie: Pertinence et faisabilité. Pharmactuel. 2014;47(2):105-13.

10. Saint-Germain P, Ruelle M, Mary A, Sid Idris S, Hannat S, Pelloquin N, et al. Impact clinique des divergences de traitement constatées chez 200 patients conciliés dans un service de gériatrie aiguë. La Revue de Médecine Interne. 2016;37(10):667-73.

11. Barthod L. Conciliation médicamenteuse à l'admission en santé mentale: cibler les patients à risque pour une démarche efficiente [Internet] [Thèse de doctorat]. [Faculté de pharmacie de Marseille] : Aix-Marseille ; 2018 [cité 04 fév 2021]. Disponible sur : https://dumas.ccsd.cnrs.fr/dumas-01834380

12. Pérennes M, Carde A, Nicolas X, Dolz M, Bihannic R, Grimont P, et al. Conciliation médicamenteuse : une expérience innovante dans un service de médecine interne pour diminuer les erreurs d'anamnèse médicamenteuses. La Presse Médicale. 2012;41(3, Part 1):e77-86. 
13. Bernard V, Rebai C, Ledoyen A, Roussel-Galle MC, Xavier-Ribot M, Beauclair P. Intégration de la conciliation médicamenteuse à une pratique pharmaceutique de terrain. Présentée à la session Hopipharm 2013. Congrès national des pharmaciens des hôpitaux. Lyon, 2013 [cité 29 nov 2020]. Disponible sur : https://www.synprefh.org/espacecongres/hopipharm/archives-hopipharm/communications-orales-affichees-lyon-2013

14. Cornish PL, Knowles SR, Marchesano R, Tam V, Shadowitz S, Juurlink DN, et al. Unintended Medication Discrepancies at the Time of Hospital Admission. Arch Intern Med. 2005;165(4):424-9.

15. Garriguet P, Beauverie P, Yassine S, Mouffak S, Alemanni J, Gaudoneix-Taïeb M, et al. Conciliation médicamenteuse en psychiatrie générale adulte: résultats de 3 années d'expérience. Le Pharmacien Hospitalier et Clinicien. 2017;52(2):138-46.

16. Boursier A, Mary A, Grados F, Desailly Henry I, Terrier Lenglet A, Fardellone P, et al. Détermination des caractéristiques des erreurs médicamenteuses détectées par la conciliation médicamenteuse en rhumatologie. Revue du Rhumatisme. 2018;85(3):285-90.

17. Decaux G. Is Asymptomatic Hyponatremia Really Asymptomatic? The American Journal of Medicine. 2006;119(7, Supplement 1):S79-82.

18. Boyer S. Contribution à l'étude du rôle de l'hyponatrémie légère et chronique dans la survenue de la chute grave du sujet âgé fragile admis en unité de Médecine d'Urgence de la Personne Agée (MUPA) [Internet] [Thèse de doctorat]. Université de Limoges ; 2018 [cité 15 nov 2020]. Disponible sur : https://www.theses.fr/2018LIMO0067

19. Renneboog B, Musch W, Vandemergel X, Manto MU, Decaux G. Mild Chronic Hyponatremia Is Associated With Falls, Unsteadiness, and Attention Deficits. The American Journal of Medicine. 2006;119(1):71.e1-71.e8.

20. Gankam Kengne F, Andres C, Sattar L, Melot C, Decaux G. Mild hyponatremia and risk of fracture in the ambulatory elderly. QJM. 2008;101(7):583-8.

21. Hoorn EJ, Rivadeneira F, van Meurs JB, Ziere G, Stricker BHc, Hofman A, et al. Mild hyponatremia as a risk factor for fractures: the Rotterdam Study. Journal of Bone and Mineral Research. 2011;26(8):1822-1828.

22. Kinsella S, Moran S, Sullivan MO, Molloy MGM, Eustace JA. Hyponatremia independent of osteoporosis is associated with fracture occurrence. Clin J Am Soc Nephrol. 2010;5(2):275-80.

23. Beuscart J-B, Petit S, Gautier S, Wierre P, Balcaen T, Lefebvre J-M, et al. Polypharmacy in older patients: identifying the need for support by a community pharmacist. BMC Geriatrics. 2019;19(1):277.

24. Ordre National des Pharmaciens. Qu'est-ce que le DP? - Le Dossier Pharmaceutique [Internet]. [cité 24 janv 2021]. Disponible sur : http://www.ordre.pharmacien.fr/LeDossier-Pharmaceutique/Qu-est-ce-que-le-DP 
Tableau 1. Analyse descriptive de la cohorte de patients ( $\mathrm{n}=136$ patients)

\begin{tabular}{lc}
\hline & $(\mathrm{n} \%)$ \\
\hline Sexe & \\
Masculin & $48(35,3)$ \\
Féminin & $88(64,7)$ \\
Age (ans, moyenne $\pm \mathrm{ET})$ & $85,9 \pm 6,8$ \\
IMC $\left(\mathrm{kg} / \mathrm{m}^{2}\right.$, moyenne $\left.\pm \mathrm{ET}\right)$ & $24,2 \pm 4,9$ \\
\hline Antécédents & \\
$\quad$ Infectieux & $15(11,0)$ \\
Cardio-vasculaires & $117(86,0)$ \\
Broncho-pulmonaires & $24(17,6)$ \\
Neurologiques & $11(8,1)$ \\
Psychiatriques & $64(47,1)$ \\
Rhumatologiques & $52(38,2)$ \\
Oncologiques & $31(22,8)$ \\
$\quad$ Digestifs & $18(13,2)$ \\
Endocrinologiques & $58(42,6)$ \\
Ophtalmologiques & $35(25,7)$ \\
Néphrologiques & $28(20,6)$ \\
Urinaires & $21(15,4)$ \\
$\quad$ Hématologiques & $15(11,0)$ \\
\hline Thrombopénie & $8(5,9)$ \\
Thrombocytose & $16(11,8)$ \\
Leucopénie & $4(2,9)$ \\
Leucocytose & $27(19,9)$ \\
Neutropénie & $3(2,2)$ \\
Polynucléose & $30(22,1)$ \\
\hline Hypoalbuminémie & $78(58,2)$ \\
Hyperalbuminémie & $1(0,7)$ \\
Hyponatrémie & $12(8,8)$ \\
Hypernatrémie & $12(8,8)$ \\
Hypokaliémie & $9(6,6)$ \\
Hyperkaliémie & $46(33,8)$ \\
Hypovitaminose D & $76(73,8)$ \\
Hypervitaminose D & $1(1,0)$ \\
\hline IRS Cockroft & $32(27,4)$ \\
IRS CDK EPI & $13(12,6)$ \\
\hline
\end{tabular}


Analyse descriptive (suite)

\begin{tabular}{lc}
\hline & $\mathrm{n}(\%)$ \\
\hline Dépendance & \\
Majeure & $67(50,4)$ \\
Totale & $6(4,5)$ \\
Troubles cognitifs & $81(59,6)$ \\
Risques de chutes & $71(65,7)$ \\
\hline Service hospitalier & $102(75,0)$ \\
dont service de chirurgie & $12(8,8)$ \\
EHPAD & $7(5,1)$ \\
Domicile & $27(19,9)$ \\
Présence d'une IDE au domicile & $86(70,5)$ \\
\hline Nombre de lignes de traitement à l'entrée & \\
Supérieures à 5 & $132(97,1)$ \\
Supérieures à 10 & $81(59,6)$ \\
Médicaments à risque & $103(75,7)$ \\
Antibiotiques & $38(36,9)$ \\
Anticoagulants & $57(55,3)$ \\
Clozapine & $2(1,9)$ \\
Neuroleptiques & $20(19,4)$ \\
Double antiagrégant plaquettaire & $6(5,8)$ \\
Méthotrexate & $2(1,9)$ \\
Anticorps monoclonal Prolia ${ }^{\circledR}$ & $2(1,9)$ \\
Anticancéreux Hydrea ${ }^{\circledR}$ & $2(1,9)$ \\
Marge thérapeutique étroite & \\
Hémigoxine & \\
Buprénorphine & $3(2,2)$ \\
Immunomodulateur Firmagon ${ }^{\circledR}$ & $1(0,7)$ \\
\hline Divergence non intentionnelle & $1(0,7)$ \\
Omission & $57(41,9)$ \\
Erreur de posologie & $48(84,2)$ \\
Erreur de dosage & $10(17,5)$ \\
Erreur de plan de prise & $4(7,0)$ \\
Erreur de médicament & 0 \\
&
\end{tabular}

Les pourcentages des médicaments à risque ont été calculés respectivement par rapport à la ligne médicaments à risque. ET : écart type ; IMC : index de masse corporelle ; IRS : insuffisance rénale sévère. 
Tableau 2. Analyse inférentielle simple

\begin{tabular}{|c|c|c|c|}
\hline & \multicolumn{2}{|c|}{$\begin{array}{c}\mathrm{n}(\%) \\
\text { Divergence non } \\
\text { intentionnelle }\end{array}$} & \multirow[t]{2}{*}{$p$} \\
\hline & Non & Oui & \\
\hline \multicolumn{4}{|l|}{ Sexe } \\
\hline Masculin & $31(64,6)$ & $17(35,4)$ & \\
\hline Féminin & $48(54,5)$ & $40(45,5)$ & 0,257 \\
\hline Age (ans, moyenne \pm ET) & $85,9 \pm 7,1$ & $85,9 \pm 6,5$ & 0,865 \\
\hline $\begin{array}{l}\mathrm{IMC}\left(\mathrm{kg} / \mathrm{m}^{2}, \text { moyenne } \pm\right. \\
\text { ET) }\end{array}$ & $24,7 \pm 5,1$ & $23,5 \pm 4,5$ & $0,190^{*}$ \\
\hline \multicolumn{4}{|l|}{ Antécédents } \\
\hline Infectieux & $9(11,4)$ & $6(10,5)$ & 0,874 \\
\hline Cardio-vasculaires & $69(87,3)$ & $48(84,2)$ & 0,603 \\
\hline Broncho-pulmonaires & $13(16,5)$ & $11(19,3)$ & 0,668 \\
\hline Neurologiques & $10(12,7)$ & $1(1,8)$ & $0,021 *$ \\
\hline Psychiatriques & $37(46,8)$ & $27(47,4)$ & 0,951 \\
\hline Rhumatologiques & $26(32,9)$ & $26(45,6)$ & $0,133^{*}$ \\
\hline Oncologiques & $16(20,3)$ & $15(26,3)$ & 0,406 \\
\hline Digestifs & $8(10,1)$ & $10(17,5)$ & 0,208 \\
\hline Endocrinologiques & $32(40,5)$ & $26(45,6)$ & 0,552 \\
\hline Ophtalmologiques & $19(24,1)$ & $16(28,1)$ & 0,597 \\
\hline Néphrologiques & $16(20,3)$ & $12(21,1)$ & 0,909 \\
\hline Urinaires & $15(19,0)$ & $6(10,5)$ & $0,178 *$ \\
\hline Hématologiques & $8(10,1)$ & $7(12,3)$ & 0,692 \\
\hline Thrombopénie & $6(7,6)$ & $2(3,5)$ & 0,468 \\
\hline Thrombocytose & $8(10,1)$ & $8(14,0)$ & 0,485 \\
\hline Leucopénie & $2(2,5)$ & $2(3,5)$ & 1,000 \\
\hline Leucocytose & $19(24,1)$ & $8(14,0)$ & $0,149 *$ \\
\hline Neutropénie & $3(3,8)$ & 0 & 0,264 \\
\hline Polynucléose & $20(25,3)$ & $10(17,5)$ & 0,281 \\
\hline Hypoalbuminémie & $45(57,7)$ & $33(58,9)$ & 0,886 \\
\hline Hyperalbuminémie & 0 & $1(1,8)$ & 0,418 \\
\hline Hyponatrémie & $2(2,5)$ & $10(17,5)$ & $0,002 *$ \\
\hline Hypernatrémie & $8(10,1)$ & $4(7,0)$ & 0,528 \\
\hline Hypokaliémie & $6(7,6)$ & $3(5,3)$ & 0,734 \\
\hline Hyperkaliémie & $23(29,1)$ & $23(40,4)$ & $0,172 *$ \\
\hline Hypovitaminose D & $44(77,2)$ & $32(69,6)$ & 0,500 \\
\hline Hypervitaminose D & $1(1,8)$ & 0 & 1,000 \\
\hline IRS Cockroft \& Gault & $21(29,2)$ & $11(24,4)$ & 0,577 \\
\hline IRS CDK EPI & $9(15,5)$ & $4(8,9)$ & 0,315 \\
\hline
\end{tabular}


Analyse inférentielle simple (suite)

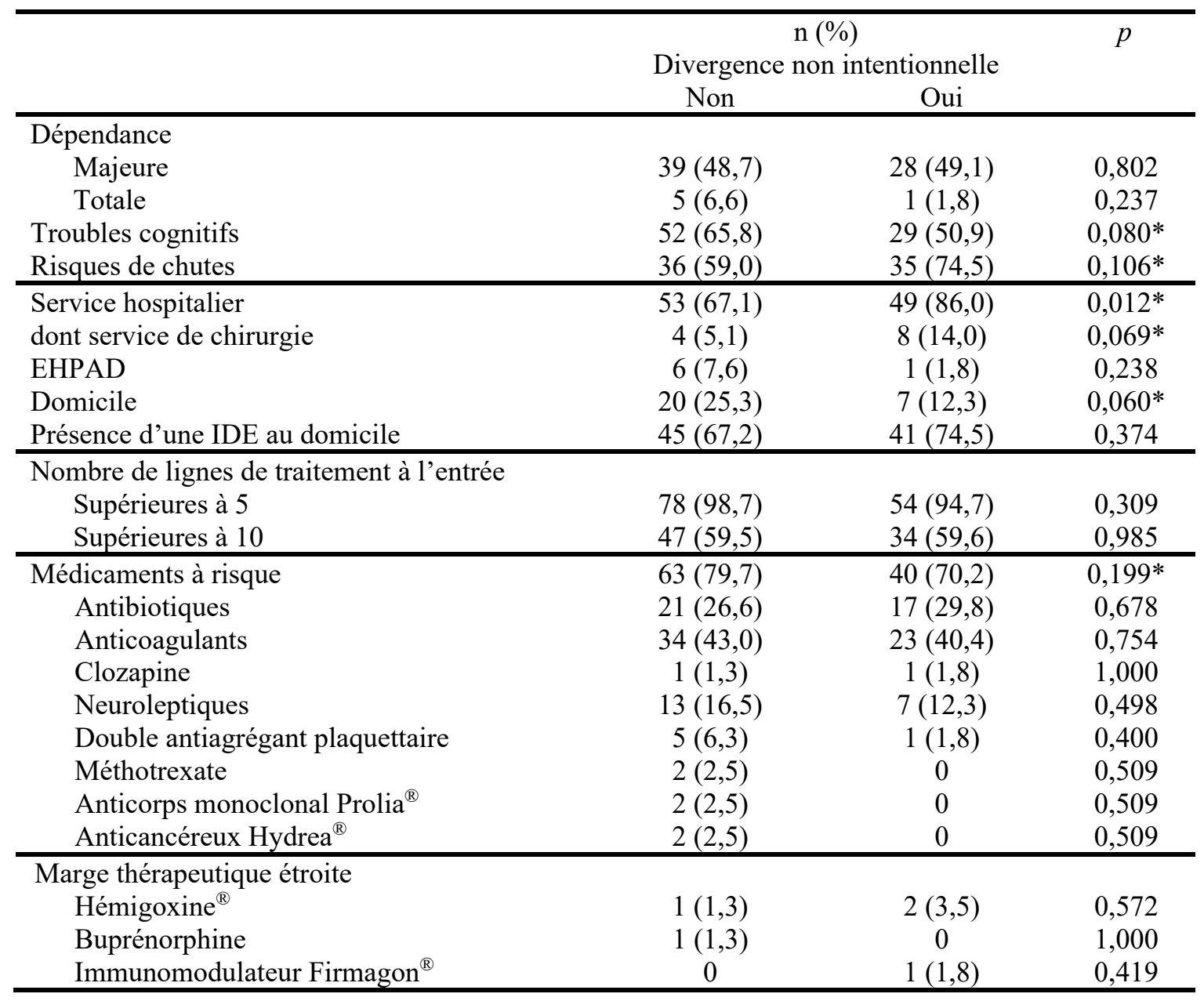

L'astérisque indique les variables utilisées pour l'analyse multiple, ET: écart type ; IMC: index de masse corporelle; IRS : insuffisance rénale sévère.

Tableau 3. Analyse inférentielle multiple

\begin{tabular}{lccc}
\hline & ORA & IC 95\% & $P$ \\
\hline Antécédents neurologiques & 0,091 & $0,011-0,779$ & 0,029 \\
Antécédents rhumatologiques & 2,276 & $1,037-4,995$ & 0,040 \\
Service hospitalier & 3,034 & $1,205-7,640$ & 0,018 \\
Hyponatrémie & 6,423 & $1,281-32,203$ & 0,024 \\
\hline
\end{tabular}

ORA : odds ratio ajusté, IC : intervalle de confiance. 
Tableau 4. Analyse détaillée

\begin{tabular}{lccc}
\hline & \multicolumn{2}{c}{$\mathrm{n}(\%)$} & $p$ \\
\hline \multicolumn{4}{c}{ Nonission } \\
\hline Antécédents neurologiques & $10(11,4)$ & $1(2,1)$ & 0,096 \\
Antécédents rhumatologiques & $30(34,1)$ & $22(45,8)$ & 0,178 \\
Service hospitalier & $61(69,3)$ & $41(85,4)$ & 0,038 \\
Hyponatrémie & $3(3,4)$ & $9(18,8)$ & 0,003 \\
\hline \multicolumn{4}{c}{ Erreur de posologie } \\
\hline Antécédents neurologiques & $10(7,9)$ & $1(10,0)$ & 0,583 \\
Antécédents rhumatologiques & $48(38,1)$ & $4(40,0)$ & 1,000 \\
Service hospitalier & $94(74,6)$ & $8(80,0)$ & 1,000 \\
Hyponatrémie & $11(8,7)$ & $1(10,0)$ & 1,000 \\
\hline \multicolumn{4}{c}{ Erreur de dosage } \\
\hline Antécédents neurologiques & $11(8,3)$ & 0 \\
Antécédents rhumatologiques & $50(37,9)$ & $2(50,0)$ & 1,000 \\
Service hospitalier & $99(75,0)$ & $3(75,0)$ & 1,037 \\
Hyponatrémie & $12(9,1)$ & 0 & 1,000 \\
\hline \multicolumn{4}{c}{ Erreur de médicament } \\
\hline Antécédents neurologiques & $11(8,1)$ & 0 \\
Antécédents rhumatologiques & $52(38,5)$ & 0 & 1,000 \\
Service hospitalier & $101(74,8)$ & $1(100,0)$ \\
Hyponatrémie & $12(8,9)$ & 0 \\
\hline
\end{tabular}

\section{Compléments électroniques :}

Complément électronique 1 : Trame entretien patient et/ou famille

Complément électronique 2 : Fiche de conciliation des traitements à l'admission

Complément électronique 3 : Bilan médicamenteux de sortie

Complément électronique 4 : Lettre explicative bilan médicamenteux de sortie jointe à la lettre de liaison d'hospitalisation

Complément électronique 5 : Détail des interventions pharmaceutiques 
COMPLEMENTS ELECTRONIQUES 



\begin{tabular}{|l|l|l|l|}
\hline & \multicolumn{3}{|l|}{ CONCILIATION MEDICAMENTEUSE } \\
& \multicolumn{4}{|c|}{ Trame entretien patient } \\
\hline
\end{tabular}

L'entretien avec le patient est une des sources importantes d'informations contribuant à la connaissance de tous les médicaments que prend le patient (prescrits ou non) et à évaluer son observance.

Il est réalisé par l'interne en pharmacie dans les premières journées d'hospitalisations puis complété, si nécessaire, lors de contacts ultérieurs avec le patient ou son entourage.

Au préalable, il consulte le dossier médical du patient afin de recueillir les premières données médicales du patient : date et motif d'hospitalisation, origine du patient (domicile, EHPAD), antécédents médicaux, traitements médicamenteux...

\section{I - INTRODUCTION}

- Se présenter, dire sa profession et expliquer l'objectif de l'entretien

- Vérifier l'identité du patient («Quel est votre nom et prénom ? » et non « Etes-vous bien $\mathrm{Mr} X ? »)+$ bracelet d'identification

- Vérifier que le patient est réceptif («Pouvez-vous me consacrer quelques minutes pour parler de votre traitement ? Ce moment vous convient-il ? »)

\section{|| - RECUEIL DES DONNEES}

Poser des questions ouvertes, par exemple :

- "Avez-vous une liste de vos médicaments ? Etes-vous venu avec vos ordonnances?"

- «Avez-vous apporté vos médicaments habituels (boîtes) ? Si oui, les avez-vous dans votre chambre ? Les prenez-vous en plus des médicaments qui vous sont donnés par les infirmières ? (Si oui, le savent-elles ?) »

- «Quels sont les médicaments que vous preniez avant votre hospitalisation ? » (Si le patient ne les connait pas : "Y a-t-il un membre proche qui connaît votre traitement ? Comment pouvons-nous le joindre ? »)

- " $\quad$ Savez-vous à quoi servent vos médicaments ?"

- "Comment prenez-vous vos médicaments ? (combien, quand, à quelle fréquence ? ex : arrêt de traitement le week-end ou prise hebdomadaire ou mensuelle ?)

- « Depuis combien de temps avez-vous ce traitement? A-t-il été modifié récemment? Si oui savez-vous pourquoi ?"

- «Avez-vous des difficultés pour les prendre (oubli, effets indésirables) ? Modifiez-vous la façon de les prendre par rapport à ce que vous a dit votre médecin et votre pharmacien ? ॥

- «Qui vous a prescrit vos médicaments ? (médecin traitant, médecins spécialistes, hospitaliers) ?» 


\section{III - CIBLAGE SUR LES MEDICAMENTS PARTICULIERS}

Insister sur les médicaments particuliers suivants et sur les modalités de suivi :

- « Est-ce que vous utilisez :

- Des gouttes pour les yeux ? gouttes/vaporisateurs pour le nez ? pommades/crèmes ?

- Des patchs ?

- Des médicaments injectables ? De l'insuline? »

- «Avez-vous des médicaments nécessitants un suivi particulier?

- Anticoagulants : avez-vous une carte patient?

- AVK : avez-vous un carnet de suivi ? Connaissez-vous votre INR cible ? »

- $\quad$ Antibiotiques: «Prenez-vous des antibiotiques ? Depuis quand et pourquoi ? En avezvous pris dans les trois derniers mois? »

- Médicaments sans prescription médicale : «Prenez-vous des médicaments en dehors de ceux prescrits par un ou plusieurs médecins que vous achetez à la pharmacie ou sur internet?»

$\rightarrow$ Phytothérapie / compléments alimentaires / vitamines / homéopathie

\section{IV - OBSERVANCE DU TRAITEMENT (si domicile)}

- "Que faites-vous lorsqu'il vous arrive d'oublier un médicament? »

- "Etes-vous aidé au quotidien dans la gestion de votre traitement ? Si oui, par qui ? "

- " Avez-vous des outils d'aide à la gestion de votre traitement ? (pilulier, alertes/alarmes téléphoniques,...)»

\section{V - ALLERGIES / EFFETS INDESIRABLES}

- «Etes-vous allergique à certains médicaments ? Comment se manifeste cette allergie ? En avez-vous déjà parlé à un professionnel de santé ?

- «Avez-vous des effets indésirables à votre traitement ? Si oui, lesquels ? »

\section{VI - COORDONNEES DES PROFESSIONNELS DE SANTE}

$\rightarrow$ Nom du médecin traitant et pharmacien d'officine. 


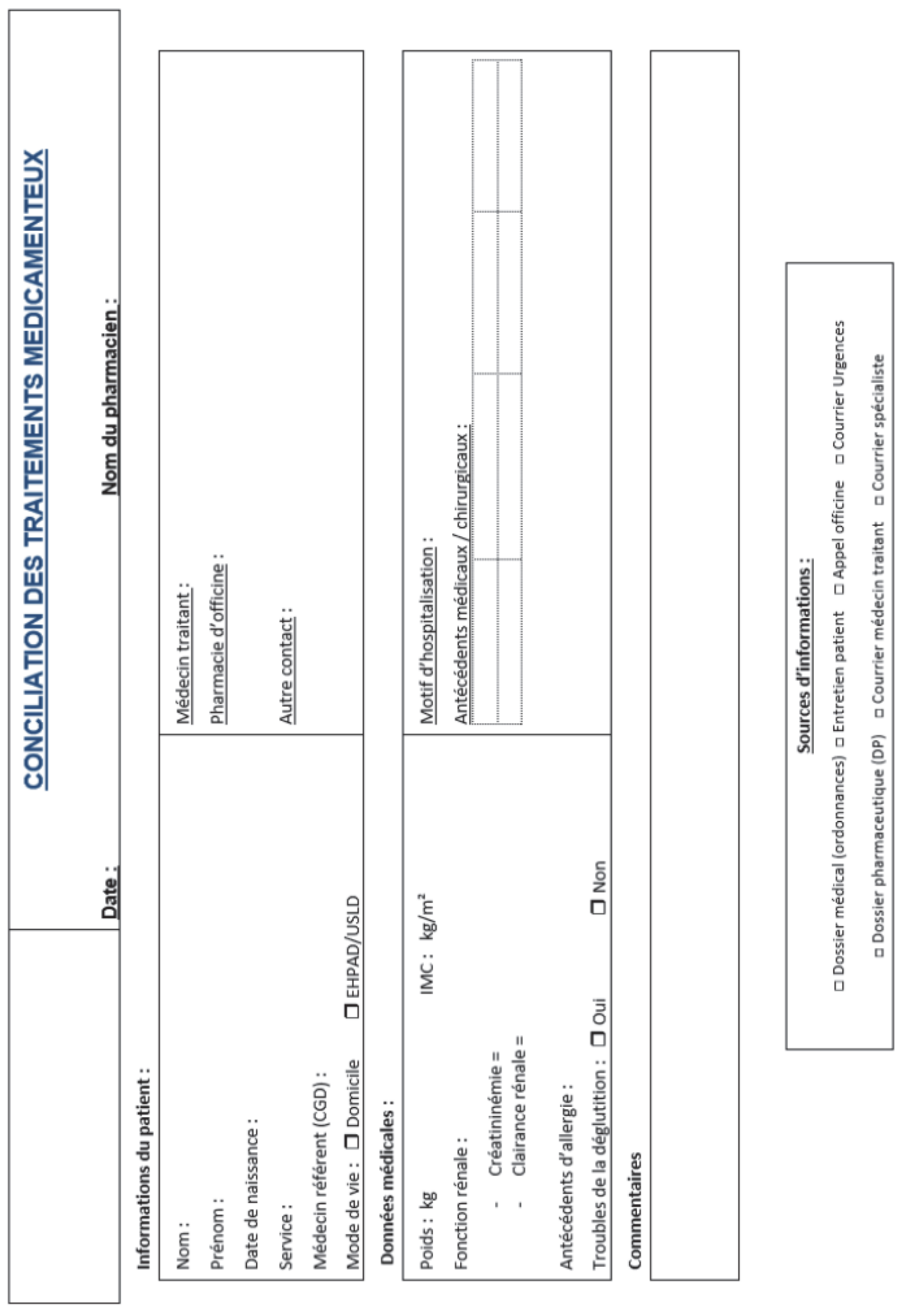




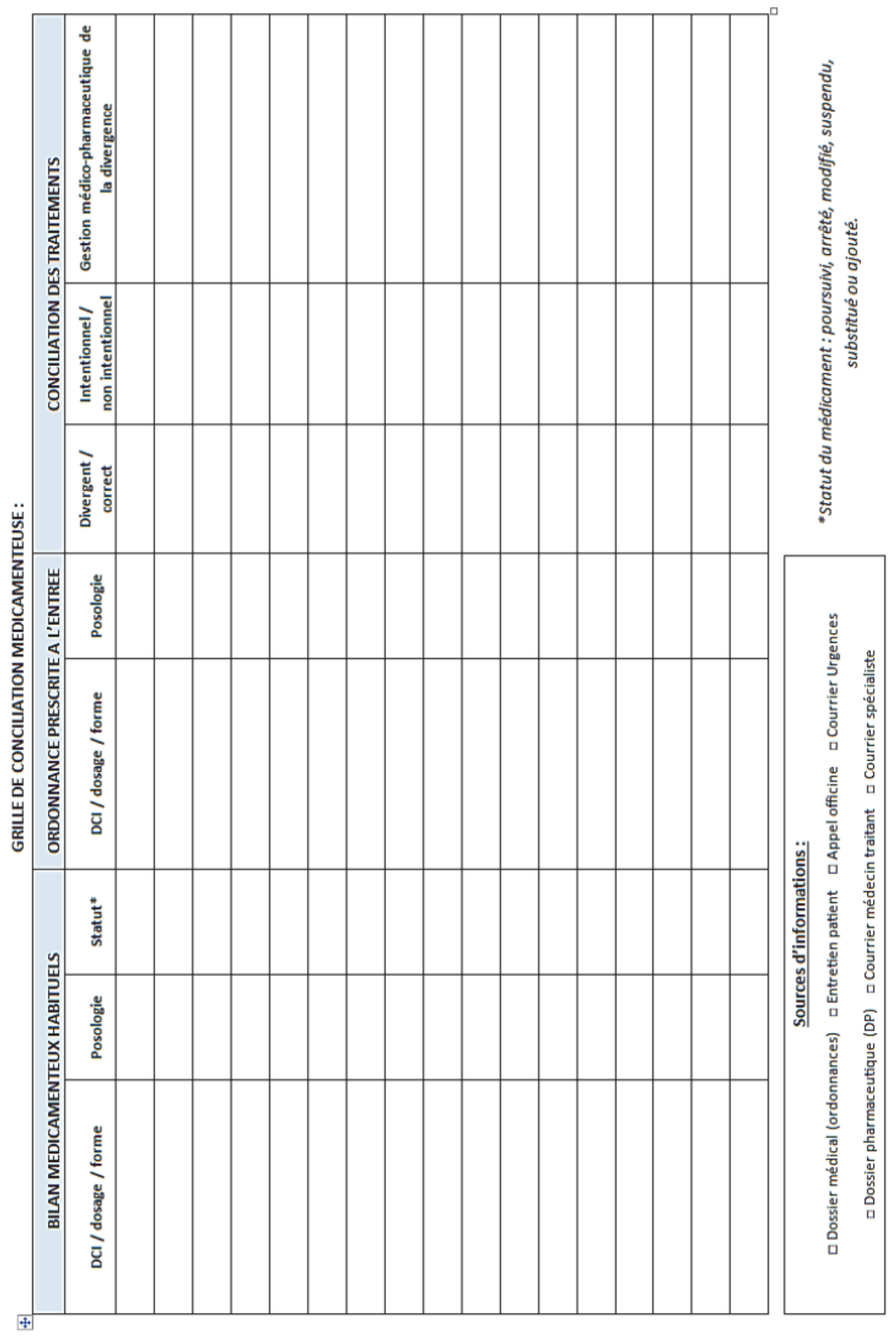


Complément électronique 3 : Bilan médicamenteux de sortie
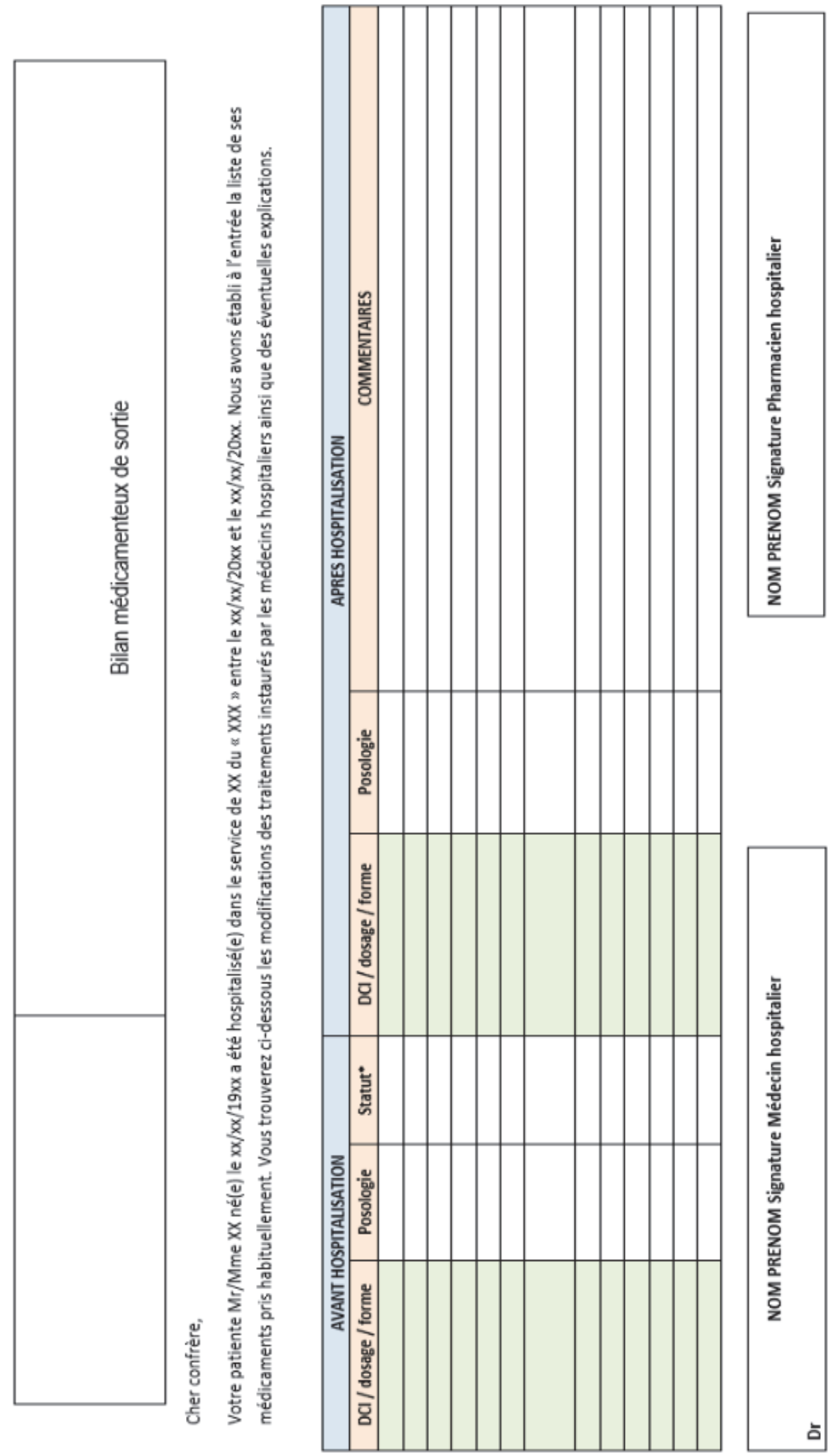
Complément électronique 4 : Lettre explicative bilan médicamenteux de sortie jointe à la lettre de liaison d'hospitalisation

\author{
Pharmacie à Usage \\ Intérieur \\ Dr \\ Dr
}

\section{EXEMPLES :}

\section{Gériatrie}

Service de Court Séjour Gériatrique

Dr

$D r$

Dr

Service de Court Séjour Alzheimer

Unité cognitivocomportementale

Dr

Dr

Dr

Service de Soins de Suite et de Réadaptation

$D r$

Dr

Dr

\section{Lettre explicative : Bilan médicamenteux de sortie}

«Date»

Chère consœur, cher confrère,

Votre patient(e), né(e) le $x x / x x / x x x x$ a été hospitalisé(e) au « XXX », dans le service de du Dr

Lors de cette hospitalisation, il ou elle a pu bénéficier d'une conciliation médicamenteuse pluriprofessionnelle (processus qui vise à sécuriser les points de transitions du circuit de soin du patient). Nous avons pris contact avec tous les professionnels de santé qui entourent votre patient(e) afin d'établir la liste exhaustive des traitements habituellement pris. Cela a permis d'avoir une ordonnance hospitalière à l'admission la plus exacte possible.

Ces traitements ont été pour certains réévalués en cours d'hospitalisation compte tenu des éléments cliniques et /ou paracliniques obtenus lors de son hospitalisation et/ou des priorités thérapeutiques. Ainsi, des traitements ont pu être modifiés, ajoutés ou supprimés.

Vous trouverez ci-joint un tableau récapitulatif mentionnant les traitements médicamenteux que nous vous proposons de poursuivre à la sortie, et les explications concernant les éventuels arrêt(s) et/ou modification(s) et/ou introduction(s) de traitement.

En espérant que ces informations vous seront utiles pour la suite de prise en charge de votre patient(e), veuillez agréer Monsieur (Madame), nos respectueuses salutations.

Bien confraternellement,

Signatures :

Docteur en pharmacie Docteur en médecine 


\section{Complément électronique 5 : Détail des interventions pharmaceutiques}

Tableau 4 : Interventions pharmaceutiques (IP) et taux d'acceptation

\begin{tabular}{|c|c|c|}
\hline IP transmises & Nombre d'IP : n & IP acceptées et prises en compte : n (\%) \\
\hline A l'entrée & 71 & $40(56,3 \%)$ \\
\hline Pendant l'hospitalisation & 69 & $48(69,6 \%)$ \\
\hline A la sortie & 3 & $2(66,7 \%)$ \\
\hline
\end{tabular}

Tableau 5 : Type d'IP transmises à l'entrée

\begin{tabular}{|c|c|c|c|}
\hline Type d'IP à l'entrée & $\begin{array}{l}\text { Nombre } \\
\text { d'IP (n) }\end{array}$ & $\begin{array}{l}\text { Classes thérapeutiques } \\
\text { les plus retrouvées }\end{array}$ & Exemple de principes actifs (PA) \\
\hline $\begin{array}{l}\text { Ajout (omission d'un } \\
\text { médicament) }\end{array}$ & 54 & $\begin{array}{c}\text { Collyre (15\%), Antidépresseur (7\%), } \\
\text { Antalgique (7\%), Vitamine D (7\%), } \\
\text { Antiasthmatique (7\%), IPP (6\%), } \\
\text { Antihistaminique (4\%) }\end{array}$ & $\begin{array}{l}\text { timoptolol, Cromedil }^{\circledR} \text {, Optive } \\
\text { fusion }^{\circledR} \text {, paroxétine, mirtazapine, } \\
\text { venlaflaxine, escitalopram, } \\
\text { paracétamol, Uvedose }^{\circledR}, \text { Zymad }^{\circledR}, \\
\text { Seretide }^{\circledR}, \text { Symbicort }^{\circledR}, \text { Innovair }^{\circledR}, \\
\text { omeprazole, pantoprazole, }^{(\text {levocetirizine }}\end{array}$ \\
\hline $\begin{array}{l}\text { Adaptation des modalités } \\
\text { d'administrations }\end{array}$ & 8 & $\begin{array}{l}\beta \text {-bloquant (25\%), Anticoagulant, } \\
\text { Antidépresseur, Anxiolytique }\end{array}$ & $\begin{array}{l}\text { sotalol, propranolol, apixaban, } \\
\text { mirtazapine, oxazepam, }\end{array}$ \\
\hline $\begin{array}{l}\text { Adaptation posologique } \\
\text { (Erreur dans le dosage } \\
\text { prescrit) }\end{array}$ & 7 & $\begin{array}{l}\text { Hormone thyroïdienne }(29 \%) \text {, } \\
\text { IPP }(29 \%) \text {, Statine, Diurétique }\end{array}$ & $\begin{array}{l}\text { Levothyrox }^{\circledR}, \text { pantoprazole, } \\
\text { atorvastatine, furosémide }\end{array}$ \\
\hline $\begin{array}{l}\text { Adaptation posologique } \\
\text { (Selon le degré d'IR) }\end{array}$ & 1 & Antidiabétique & metformine \\
\hline $\begin{array}{c}\text { Substitution } \\
\text { (Erreur de PA) }\end{array}$ & 1 & Antiangoreux & nicorandil \\
\hline
\end{tabular}

IP : intervention pharmaceutique ; IPP : inhibiteur de la pompe à protons ; IR : insuffisance rénale 


\begin{tabular}{|c|c|c|c|}
\hline $\begin{array}{c}\text { Type d'IP pendant } \\
\text { l'hospitalisation }\end{array}$ & $\begin{array}{l}\text { Nombre } \\
\text { d'IP (n) }\end{array}$ & $\begin{array}{c}\text { Classes thérapeutiques } \\
\text { les plus retrouvées }\end{array}$ & Exemple de principes actifs (PA) \\
\hline $\begin{array}{l}\text { Adaptation posologique } \\
\text { (Selon le degré d'IR) }\end{array}$ & 22 & $\begin{array}{c}\text { Antidiabétique (18\%), antalgique } \\
(18 \%) \text {, anticoagulant injectable } \\
(9 \%) \text {, antibiotique }(9 \%), \\
\text { antihistaminique }\end{array}$ & $\begin{array}{l}\text { metformine, paracétamol, Lovenox }{ }^{\circledR} \text {, } \\
\text { ciprofloxacine, ertapénem, cétirizine }\end{array}$ \\
\hline Suivi thérapeutique (kaliémie) & 10 & $\begin{array}{l}\text { Médicament potentialisant le } \\
\text { risque d'hyperkaliémie }\end{array}$ & $\begin{array}{c}\text { candesartan, périndopril, Movicol }^{\circledR} \text {, } \\
\text { Aldactone }{ }^{\circledR} \\
\end{array}$ \\
\hline Arrêt (redondance de PA) & 6 & $\begin{array}{c}\text { Antalgique, IPP, vitamine D, } \\
\text { AVK, calcium }\end{array}$ & $\begin{array}{c}\text { paracétamol, pantoprazole, Uvedose }{ }^{\circledR} \text {, } \\
\text { fluindione, Calcidose }{ }^{\circledR}\end{array}$ \\
\hline $\begin{array}{c}\text { Arrêt } \\
\text { (contre-indication si IRS) }\end{array}$ & 5 & $\begin{array}{l}\alpha \text {-bloquant (40\%), antigoutteux, } \\
\text { statine, anti-hypertenseur }\end{array}$ & $\begin{array}{l}\text { alfuzosine, colchicine, } \\
\text { rosuvastatine, rilménidine }\end{array}$ \\
\hline $\begin{array}{l}\text { Adaptation posologique } \\
\text { (erreur de posologie) }\end{array}$ & 5 & $\begin{array}{l}\text { Anticoagulant injectable (60\%) } \\
\text { et per os, sartan }\end{array}$ & $\begin{array}{c}\text { Innohep }^{\circledR} \text { curatif, Lovenox }{ }^{\circledR} \text {, apixaban, } \\
\text { candesartan }\end{array}$ \\
\hline $\begin{array}{c}\text { Adaptation posologique } \\
\text { (erreur unité de prescription) }\end{array}$ & 5 & $\begin{array}{l}\text { Anticoagulant injectable }(60 \%), \\
\text { laxatif, antibiotique }\end{array}$ & $\begin{array}{l}\text { Fraxodi }^{\circledR}, \text { Calciparine }^{\circledR}, \text { Movicol }^{\circledR} \text {, } \\
\text { méropénème }\end{array}$ \\
\hline $\begin{array}{c}\text { Adaptation posologique } \\
\text { (diminution posologie selon } \\
\text { l'AMM) }\end{array}$ & 4 & Antalgique $(75 \%)$, antibiotique & paracétamol, gentamicine \\
\hline $\begin{array}{c}\text { Optimisation des modalités } \\
\text { d'administrations } \\
\text { (plan de prise selon l'AMM) }\end{array}$ & 3 & $\begin{array}{l}\text { Anticalcique, antiangoreux, } \\
\text { neuroleptique injectable }\end{array}$ & $\begin{array}{c}\text { diltiazem, Trinipatch } \\
\text { injectable } \\
{ }^{\circledR}, \text { Zyprexa }^{\circledR}\end{array}$ \\
\hline $\begin{array}{c}\text { Substitution /échange } \\
\text { (adaptation au livret } \\
\text { thérapeutique) }\end{array}$ & 3 & Sartan, anticoagulant injectable & candesartan, Innohep $^{\circledR}$ \\
\hline $\begin{array}{l}\text { Adaptation posologique } \\
\text { (mention de non-sécabilité du } \\
\text { comprimé) }\end{array}$ & 2 & Anticancéreux, AVK & méthotrexate, coumadine \\
\hline $\begin{array}{c}\text { Suivi thérapeutique } \\
\text { (association déconseillée) }\end{array}$ & 2 & $\begin{array}{c}\text { Neuroleptique }+ \text { antidépresseur, } \\
\text { antiangoreux }+ \text { antiagrégant } \\
\text { plaquettaire }\end{array}$ & 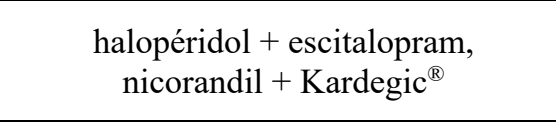 \\
\hline $\begin{array}{l}\text { Suivi thérapeutique } \\
\text { (allergie) }\end{array}$ & 2 & Antibiotique & ceftriaxone \\
\hline $\begin{array}{c}\text { Ajout } \\
\text { (instauration selon indication) }\end{array}$ & 1 & AVK & fluindione \\
\hline
\end{tabular}

IP : intervention pharmaceutique ; IR : insuffisance rénale, $\mathrm{AVK}=$ antivitamine $\mathrm{K}$; IRS $=$ insuffisance rénale sévère ; AMM : autorisation de mise sur le marché

Tableau 7 : Types d'IP transmises à la sortie

\begin{tabular}{|c|c|c|c|}
\hline Type d'IP à la sortie & $\begin{array}{l}\text { Nombre } \\
\text { d'IP (n) }\end{array}$ & Classes thérapeutiques & Principes actifs (PA) \\
\hline $\begin{array}{l}\text { Adaptation posologique } \\
\text { (Selon le degré d'IR) }\end{array}$ & 3 & $\begin{array}{c}\text { Anticoagulant injectable, Diurétique, } \\
\text { Statine }\end{array}$ & $\begin{array}{c}\text { Lovenox }^{\circledR}, \text { hydrochlorothiazide, } \\
\text { pravastatine }\end{array}$ \\
\hline
\end{tabular}

IR : insuffisance rénale 
DISCUSSION GENERALE ET PERSPECTIVES 

La population gériatrique est particulièrement exposée aux risques d'EIM. En effet, les sujets âgés sont le plus souvent atteints de différentes pathologies donc polymédiqués et sujets à un risque de iatrogénie médicamenteuse. Les équipes médicales doivent être particulièrement attentives au rapport bénéfice/risque des MPI (anticholinergiques, neuroleptiques, hypnotiques, benzodiazépines à longue demi-vie, tramadol ...) [63]. De plus, le risque de rupture de suivi de traitement et d'automédication par le patient en ville impose un certain niveau de vigilance dans la réévaluation des prescriptions lors de toute hospitalisation.

Dans cette optique, la CTM est devenue une priorité de la HAS qui impose de l'étendre et de la généraliser au sein des ES par le biais des certifications et notamment celle de 2020 (critère 2.2.1-06). Cette activité est également devenue une des missions principales du pharmacien hospitalier puisqu'elle est inscrite dans le nouveau décret des PUI n²019-489 de mai 2019 et analysée au niveau national grâce à l'indicateur Q7 du CAQES. Son enjeu principal est de prévenir ou corriger les EM potentiellement iatrogènes de façon pluridisciplinaire.

Afin d'optimiser au mieux cette activité chronophage, les OMEDIT proposent des critères de priorisation des patients tels que l'âge, certaines comorbidités (diabète, cancer, ...) ou la présence de médicaments à risque sur l'ordonnance d'entrée. Cependant, ceux-ci ne sont potentiellement pas adaptés à une population exclusivement gériatrique, ce qui est le cas précis de notre étude. Il était donc intéressant de définir des critères de priorisation pertinents grâce à une analyse statistique d'une cohorte de patients âgés.

L'objectif était donc d'évaluer des facteurs prédictifs de risque de présence de DNI sur l'ordonnance d'entrée du patient hospitalisé lors de la conciliation médicamenteuse d'entrée. Elle a été réalisée sur une cohorte de 136 patients conciliés à l'entrée de leur hospitalisation dans des services de courts ou moyens séjours. Au total, 63 DNI ont été recensées dont la majeure partie impliquait des omissions de médicament $(76,2 \%)$, suivies des erreurs de posologie $(15,9 \%)$ et de dosage $(6,3 \%)$ et de PA (1,6\%). Le pourcentage observé de DNI $(46,3$ $\%$ ) de notre étude se situe dans la moyenne par rapport aux résultats d'autres études répertoriant, en moyenne, de $32 \%$ à $54 \%$ de DNI à l'admission [106-110].

D'après l'analyse statistique de notre étude, trois critères ont été retenus comme facteurs prédictifs du risque de DNI sur l'ordonnance d'entrée par rapport au BMO. Les antécédents rhumatologiques, la provenance d'un service hospitalier et l'hyponatrémie apparaissaient comme facteurs prédictifs significatifs $(\mathrm{p}<0,5)$ alors que des antécédents neurologiques étaient prédicteurs indépendants d'une faible incidence de DNI (ORA $=0,091 ; p=0,029)$. 
La provenance d'un service hospitalier engendre une multiplicité des prescriptions et un manque de transfert d'informations entre les ES, d'où le risque d'omission de traitement. Le résultat des antécédents rhumatologiques avec le risque de chute lié à une iatrogénie médicamenteuse parait cohérent chez le sujet âgé. L'hyponatrémie, désordre électrolytique le plus fréquemment rencontré en gériatrie, est un facteur de risque de chute et de syndrome confusionnel [111]. Une perte de mémoire pendant l'entretien peut donc expliquer le lien entre la présence d'une hyponatrémie à l'entrée et l'existence d'une DNI à type d'omission d'un traitement. De plus, cet effet indésirable peut être retrouvé lors de l'usage de multiples médicaments fréquemment prescrits en ville et peu réévalués, le lien avec l'existence d'une DNI à l'entrée parait donc cohérent (absence de suivi thérapeutique).

A l'avenir et sous couvert de l'accord des équipes médicales en COMEDIMS, cette étude permettra d'envisager plusieurs améliorations dans la procédure interne de conciliation médicamenteuse.

Dans un premier temps, les critères de priorisation des patients à concilier seront modifiés au vu des résultats obtenus. Le choix des patients pourra donc être optimisé grâce à la création d'une fiche de recueil de données remplie par l'interne en pharmacie permettant de relever à chaque entrée : la provenance du patient, la présence d'une hyponatrémie sur le bilan biologique d'entrée et la présence d'antécédents rhumatologiques. Ceci permettra en parallèle de répondre à l'indicateur national Q7 du CAQES «Nombre de patients priorisés et bénéficiant d'une conciliation médicamenteuse d'entrée et/ou de sortie / Nombre de patients priorisés hospitalisés » [91]. Jusqu'ici, cet indicateur était calculé grâce à un audit réalisé sur une période donnée (un mois) en extrapolant le résultat pour un an, comme proposé par plusieurs OMEDIT. A présent, il sera possible de le calculer de façon annuelle et d'avoir un résultat plus juste et cohérent. La mise en place d'une évaluation des pratiques professionnelles (EPP) sera alors envisageable pour suivre l'évolution de l'activité de CTM d'année en année et ainsi améliorer nos pratiques.

Dans un second temps, d'autres perspectives pourraient être envisagées en travaillant avec des services transversaux de l'établissement: la création d'un sigle dans le DPI si le patient est priorisé pour une CTM (accord du médecin plus rapide) ; alerte par le laboratoire de biologie médicale en cas d'hyponatrémie retrouvée à l'entrée du patient; mise en place de l'accès au dossier pharmaceutique (DP) dans l'établissement. 
Concernant ce dernier point, la généralisation de l'accès au DP pour les médecins exerçant en ES a officiellement été lancée en octobre 2017 suite au décret du 9 mai 2017 et de l'autorisation de la Commission Nationale de l'Informatique et des Libertés (CNIL). Cet accès est effectivement à optimiser afin de bénéficier d'une source d'information supplémentaire fiable et de contribuer à la coordination entre professionnels de santé et au lien ville-hôpital. D'après l'étude de Henry et al., 192 DI et 23 DNI ont été relevées. Dans le groupe « non DP », 2 DNI ont été détectées. Dans le groupe « DP », 21 DNI ont été détectées (soit $91 \%$ des DNI totales) dont 20 grâce au DP confronté à une autre source. Parmi ces 20 DNI, 10 n'auraient pas pu être détectées en l'absence de consultation du DP (50\%). Ces 20 DNI résultaient de l'omission d'un médicament sur l'OMA $(\mathrm{n}=10)$ ou d'un dosage erroné $(\mathrm{n}=10)$ [112]. D'après l'étude de Gutermann et al., suite à la mise en place du DP, le pourcentage de CTM à l'admission réalisées à partir d'au moins 3 sources a significativement augmenté (38 \% vs $51 \%$ ) [113]. En le confrontant à d'autres sources, le DP constitue donc une source très intéressante à la détection des EM. Et ce, d'autant plus, chez les patients ne connaissant pas leur traitement ou ayant un dossier médical très peu renseigné $[112,113]$. Nous prévoyons alors de signer une convention avec le Conseil national de l'Ordre des pharmaciens pour se connecter au DP des patients [114].

Afin de renforcer là encore le lien ville-hôpital, nous envisageons de réaliser des études de suivi des CTM de sortie (prises en compte des modifications thérapeutiques au cours de l'hospitalisation par le médecin traitant, transfert efficient de ces modifications thérapeutiques à la pharmacie d'officine). En effet, le bilan médicamenteux de sortie (BMS) réalisé lors de la CTM de sortie est intégré au compte rendu d'hospitalisation et transféré à la pharmacie d'officine référente du patient. Un appel à la pharmacie d'officine et au patient, 1 mois après sa sortie d'hospitalisation pourrait être effectué afin de vérifier la concordance avec le traitement de sortie d'hospitalisation. Si des divergences sont identifiées, une analyse de celles-ci avec contact au médecin traitant pourrait être envisagée afin d'en déterminer la caractéristique (intentionnelle ou non intentionnelle). D'après l'étude réalisée par Moulis-Rivière [115], avec l'envoi du BMS aux médecins traitants, 88,5 \% des optimisations thérapeutiques réalisées sont poursuivies après la sortie. Le BMS a donc un effet bénéfique sur le maintien de l'optimisation thérapeutique après la sortie.

Enfin, le projet de notre établissement est d'étendre la CTM à l'HDJ gériatrique. L'objectif est de faire bénéficier au patient d'une évaluation gérontologique pluridisciplinaire pour établir un diagnostic et assurer une thérapeutique adaptée au sujet âgé en évitant de recourir à une hospitalisation classique. Au décours de cette journée, le pharmacien peut être amené à 
intervenir sur le sujet de la thérapeutique du patient lors d'un entretien pharmaceutique : optimisations du traitement (adaptations posologiques, substitutions de traitements si présence de MPI, ...), conseils dans la préparation ou l'administration de traitements. En parallèle, une CTM d'entrée pourra être réalisée de manière proactive (recueil des informations avant l'HDJ) afin d'avoir un regard complet et exhaustif sur les traitements prescrits ou non du patient. Le pharmacien finalisera ensuite les documents de sortie du patient : BMS selon les modifications faites par le médecin de l'HDJ à l'attention du médecin traitant ; plan de prise personnalisé à l'attention du patient et/ou des aidants (si nécessaire). Dans la littérature, d'après l'étude de Jean-Bart et al., un suivi pharmaceutique a été réalisé en HDJ pour 67 patients âgés. Parmi eux, $34 \%$ avaient des MPI [116]. L'étude de Degeorges et al., a retrouvé 61 IP au cours de la CTM en HDJ qui ont permis la suppression de 14 MPI (22,95\% des cas) [117]. Dans un souci d'optimisation du temps pharmaceutique et de pertinence, les critères de priorisation des patients obtenus dans notre étude principale pourront être également utilisés dans la mise en place de la CTM dans le secteur émergent de l'ambulatoire, situé entre la ville et l'hôpital. Pour finir, une grande partie des patients conciliés étant en provenance d'un service hospitalier, il serait intéressant d'étudier dans une prochaine étude le type de DNI retrouvées selon la spécificité des services d'où proviennent les patients (urgences, unité d'hospitalisation de courte durée, chirurgie, médecine, ...). 
CONCLUSIONS 

La CTM, processus interactif et pluriprofessionnel, garantit la continuité des soins [5]. Elle représente une obligation réglementaire de la HAS également depuis le nouveau décret PUI (n²019-489) de mai 2019 avec les activités de pharmacie cliniques et apparaît dans les indicateurs du CAQES. L'enjeu principal est de prévenir ou corriger les EM potentiellement iatrogènes, en favorisant la transmission d'informations complètes et exactes sur les médicaments du patient aux points de transition qui sont le plus souvent à risque médicamenteux tels que l'admission, la sortie et les transferts du patient [6]. Des critères de priorisation à la réalisation de la CTM pour les patients hospitalisés ont été proposés par plusieurs OMEDIT [8-10] mais non adaptés pour une population exclusivement gériatrique qui est particulièrement exposée aux risques d'EIM.

L'objectif de ce travail était donc d'analyser et de définir des critères de priorisation pertinents pour la CTM en gériatrie afin d'éviter le maximum d'EM et de sensibiliser les patients et les professionnels de santé à l'intérêt de cette activité.

Dans un premier temps, un audit clinique a été réalisé de façon prospective dans un établissement gériatrique des Hôpitaux de Provence sur une durée de 10 mois, de juin 2019 à mars 2020. Il s'intéressait à l'activité de CTM réalisée aux points de transition spécifiques que sont l'admission et la sortie du patient. En accord avec les équipes médicales, les patients à risque d'EM étaient prioritaires pour la CTM s'ils présentaient un des trois critères suivants : admission d'un service hospitalier, IRS et prescription de médicaments à risque.

Pendant la durée de l'étude, 151 patients (94 femmes et 57 hommes) étaient priorisés selon les critères définis donc théoriquement à concilier. Au total, $138 \mathrm{CTM}$ ont été réalisées : 108 à l'entrée et la sortie, 28 uniquement à l'entrée et 2 uniquement à la sortie du patient.

La cohorte de patients conciliés à l'entrée comprenait donc 136 patients avec un ratio H/F de 0,55 ( 88 femmes et 48 hommes) et une moyenne d'âge de 85,9 ans ( \pm 5,23 ans). Les antécédents cardio-vasculaires représentaient la majorité des antécédents des patients (86\%) suivis des antécédents psychiatriques (47,1\%), endocrinologiques $(42,6 \%)$ et rhumatologiques $(38,2 \%)$. Le nombre de lignes de traitement à l'entrée était supérieur à 5 pour 97,1\% des patients et supérieur à 10 pour $59,6 \%$ des patients, avec une moyenne de 10 lignes par patient $( \pm 3)$. Dans notre étude, $75,7 \%$ des patients avaient au moins un médicament à risque présent sur son ordonnance d'entrée, notamment des anticoagulants (55,3\%), des antibiotiques (36,9\%) et des neuroleptiques $(19,4 \%)$. 
En moyenne, 9 divergences (DI ou DNI) ont été retrouvées par prescription à l'entrée du patient. Les DI étaient de 8 en moyenne par prescription, avec une médiane de 8 (entre 1 et 18). La réévaluation du traitement habituel du patient par les équipes médicales hospitalières était donc appliquée et nécessaire.

Cependant, notre étude a retrouvé une moyenne de 0,8 DNI par prescription. Celles-ci correspondaient à des modifications de traitement involontaires ou EM (ordonnance non transmise, omission de médicament, erreurs de posologie, de voie d'administration ou d'horaire de prise). Au total, 63 DNI ont été recensées pendant la durée de notre étude dont la majeure partie impliquait des omissions de médicament (76,2\%).

D'après l'ensemble de l'analyse statistique, trois critères ont été retenus comme facteurs prédictifs indépendants du risque de DNI sur l'ordonnance d'entrée par rapport au BMO : provenance d'un service hospitalier, hyponatrémie à l'entrée et antécédents rhumatologiques du patient.

La provenance d'un service hospitalier engendre une multiplicité des prescriptions et un manque de transfert d'informations entre les ES, d'où le risque d'omission de traitement. Les antécédents rhumatologiques s'expliquent par le risque de chute lié à une iatrogénie médicamenteuse chez le sujet âgé. Quant au résultat de l'hyponatrémie, elle peut causer un syndrome confusionnel [111] d'où l'existence d'une DNI à type d'omission d'un traitement chez ces patients. De plus, cet effet indésirable peut être retrouvé lors de l'usage de multiples médicaments fréquemment prescrits en ville et peu réévalués.

Notre travail s'avère être une étude complète dotée d'un suivi précis du patient avec notamment l'analyse des IP réalisées tout au long du parcours du patient. Cependant, l'utilisation du DP aurait permis d'avoir un recueil plus exhaustif des données.

A l'avenir, cette étude permettra d'envisager une modification des critères de priorisation sur la procédure de l'ES ainsi que la mise en place de la CTM en HDJ gériatrique afin de renforcer le lien ville-hôpital. 


\section{REFERENCES BIBLIOGRAPHIQUES}

1. INSEE. Population par âge, Tableaux de l'économie française. 2018 [Internet]. [cité 11 nov 2020]. Disponible sur : https://www.insee.fr/fr/statistiques/3303333? sommaire $=3$ 353488

2. INSEE. Bilan démographique. 2019 [Internet]. [cité 11 nov 2020]. Disponible sur : https://www.insee.fr/fr/statistiques/4281618\#titre-bloc-21

3. Legrain S, Lacaille S. Prescription médicamenteuse du sujet âgé. EMC - Médecine. 2005;2(2):127-36.

4. Etablissements Public de Santé Mentale - Agglomération Lilloise. La conciliation médicamenteuse : une nouvelle activité mise en place au CPAA [Internet]. [cité 11 nov 2020]. Disponible sur : https://www.epsm-al.fr/article/la-conciliation-medicamenteuseune-nouvelle-activite-mise-en-place-au-cpaa

5. OMEDIT Ile de France. Conciliation médicamenteuse [Internet] [cité 11 nov 2020]. Disponible sur : http://www.omedit-idf.fr/qualite-securite/parcours-du-patient/concil iation-medicamenteuse-2/

6. HAS. Mettre en œuvre la conciliation des traitements médicamenteux en établissement de santé [Internet]. [cité 11 nov 2020]. Disponible sur : https://www.hassante.fr/jcms/c_2736453/fr/mettre-en-oeuvre-la-conciliation-des-traitements-medica menteux-en-etablissement-de-sante

7. JORF. Décret $n^{\circ}$ 2019-489 du 21 mai 2019 relatif aux pharmacies à usage intérieur. [Internet]. [cité 11 nov 2020]. Disponible sur : https://www.legifrance.gouv.fr/loda /id/JORFTEXT000038496476/

8. OMEDIT Centre, Partage d'expériences régionales sur l'activité de conciliation médicamenteuse. [Internet]. [cité 11 nov 2020]. Disponible sur : http://www.omeditcentre.fr/portail/gallery_files/site/136/2953/4197/4829/7960/7975.pdf

9. OMEDIT Normandie, Guide d'utilisation outils de priorisation en pharmacie clinique version avril 2018. [Internet]. [cité 11 nov 2020]. Disponible sur : http://www.omeditnormandie.fr/media-files/12223/guide-d-utilisation-outils-de-priorisation-enpharmacie-clinique-version-avril-2018-1-.pdf

10. OMEDIT Centre. Focus sur la conciliation médicamenteuse [Internet]. [cité 11 nov 2020]. Disponible sur : http://www.omedit-centre.fr/portail/gallery_files /site/136/2953/3607/4888/8093.pdf

11. Lannoy D. EPU pharmaciens hospitaliers. La iatrogénie médicamenteuse [Internet]. [cité 8 nov 2020]. Disponible sur : http://www.aphnep.org/pdf/Journee/2012/8iatrogenie.pdf

12. HAS. La iatrogénie médicamenteuse chez le sujet âgé, Rencontre régionale sur la certification des établissements de santé Hauts de France 2017. [cité 8 nov 2020]. Disponible sur : https://www.has-sante.fr/upload/docs/application/pdf/2017-12/rencon tre_regional_ces_-_groupe_ahnac_polyclinique_de_riaumont_iatrogenie.pdf 
13. Bedouch $\mathrm{P}$, Bardet J-D, Chanoine $\mathrm{S}$, Allenet $\mathrm{B}$. Iatrogénèse médicamenteuse : quels enjeux pour la pharmacie clinique? Pharmacie clinique et thérapeutique Elsevier Masson. 2018;7-17.

14. VIDAL. Iatrogénie médicamenteuse chez les personnes âgées : enquête, campagne de sensibilisation et actions annoncées du Leem [Internet]. [cité 8 nov 2020]. Disponible sur: https://www.vidal.fr/actualites/15365/iatrogenie_medicamenteuse_chez_les_personnes_a gees_enquete_campagne_de_sensibilisation_et_actions_annoncees_du_leem/

15. Société française de pharmacie clinique. Dictionnaire français de l'erreur médicamenteuse [Internet] 2006 [cité 08 nov 2020]. Disponible sur : http://optimiz-sihcirc-med.fr/Documents/Dictionnaire_SFPC_EM.pdf

16. Laborde M. Impact de la conciliation pharmaceutique à l'entrée et à la sortie du patient hospitalisé en diabétologie. [Internet] [Thèse de doctorat]. Faculté de pharmacie de Toulouse III Paul Sabatier ; 2016 [cité 8 nov 2020]. Disponible sur : http://thesesante.ups-tlse.fr/1326/1/2016TOU32039.pdf

17. CSP. Article R. 5121-153 du Code de la santé publique, mis en conformité à la directive 2000/38/CE du 5 juin 2000 par le décret $n^{\circ} 2004-99$ du 29 janvier 2004 relatif à la pharmacovigilance [Internet]. [cité 8 nov 2020]. Disponible sur : https://www.legi france.gouv.fr/codes/section_lc/LEGITEXT000006072665/LEGISCTA000006190672/ 2012-11-10/\#LEGISCTA000006190672

18. Michel P, Quenon JL, Djihoud A, TricaudVialle S, de Sarasqueta AM, Domecq C et al. Les événements indésirables graves liés aux soins observés dans les établissements de santé : premiers résultats d'une étude nationale. Études et résultats, DREES, Paris. 2005;398:1-15.

19. Pourcelot C, Peter V, Alpy-Girard M, Barnoux M. Mise en place d'une consultation pharmaceutique lors de la dispensation des médicaments en rétrocession. Le Pharmacien Hospitalier et Clinicien. 2017;52(1):e26.

20. Olivier P, Caron J, Haramburu F, Imbs J-L, Jonville-Béra A-P, Lagier G, et al. Validation d'une échelle de mesure: exemple de l'échelle française d'évitabilité des effets indésirables médicamenteux. Therapies. 2005;60(1):39-45.

21. NCCMERC (National Coordinating Council for Medication Error Reporting and. About Medication Errors [Internet]. 2014 [cité 8 nov 2020]. Disponible sur : https://www.nccmerp.org/about-medication-errors

22. ANSM : Agence nationale de sécurité du médicament et des produits de santé. Qu'est-ce qu'une erreur médicamenteuse? [Internet]. [cité 30 nov 2020]. Disponible sur : https://www.ansm.sante.fr/Declarer-un-effet-indesirable/Erreurs-medicamenteuses/Quest-ce-qu-une-erreur-medicamenteuse/(offset)/0

23. Arnaud A. La conciliation médicamenteuse en psychiatrie, expérience pratique au centre hospitalier Gérard Marchant. [Internet] [Thèse de doctorat]. Faculté de pharmacie de Toulouse III Paul Sabatier ; 2016 [cité 8 nov 2020]. Disponible sur : http://thesesante.ups-tlse.fr/1731/1/2017TOU32111.pdf 
24. Ferchichi S, Antoine V. Le bon usage des médicaments chez la personne âgée. La Revue de Médecine Interne. 2004;25(8):582-90.

25. ANSM. Prévenir la iatrogénèse médicamenteuse chez le sujet âgé [Internet]. [cité 12 nov 2020]. Disponible sur : https://ansm.sante.fr/var/ansm_site/storage/original/ application/9641eb3f4a1e67ba18a6b8aecd3f1985.pdf

26. HAS. Manuel de certification des établissements de santé V2010 [Internet]. [cité 12 nov 2020]. Disponible sur : https://www.has-sante.fr/upload/docs/application/pdf/201403/manuel_v2010_janvier2014.pdf

27. Réalités cardiologiques, 2012, Hypertension artérielle du sujet âgé : comment traiter sans être iatrogène? [Internet]. [cité 15 nov 2020]. Disponible sur : http://www.realitescardiologiques.com/wp-content/uploads/sites/2/2012/01/081.pdf

28. Macquin-Mavier I. Capacité de gérontologie - mars 2007. Pharmacologie et sujets âgés. [Internet]. [cité 14 nov 2020]. Disponible sur : http://134.157.193.4/polys/ capacites/capagerontodocs/annee2therapeuthique/pharmacologiegeneraleprmacquinma vier2007.pdf

29. Joël Ankri, Risque iatrogène et population âgée, Médicament et risque adsp $n^{\circ} 27$ juin 1999, pp 41-43.

30. Motte C. Optimisation diagnostique et thérapeutique chez la personne âgée : application de l'outil PMSA (Prescription Médicamenteuse chez la Personne Agée) dans un service de court séjour gériatrique d'entrée directe [Internet] [Thèse de doctorat]. Faculté de médecine de Nancy ; 2016 [cité 24 janv 2021]. Disponible sur : https://hal.univlorraine.fr/hal-01932232/document

31. Prudent M. Iatrogénie associée à la prescription de psychotropes chez les sujets âgés [Internet] [Thèse de doctorat]. Université de Reims ; 2017 [cité 15 nov 2020]. Disponible sur : https://www.theses.fr/2017REIMM204

32. Pharmacie-clinique. Evaluation de la charge anticholinergique : Quelle échelle utiliser en pratique clinique? [Internet]. 2019 [cité 18 janv 2021]. Disponible sur : http://pharmacie-clinique.fr/evaluation-de-la-charge-anticholinergique-quelle-echelleutiliser-en-pratique-clinique/

33. Le T. Anticholinergic effects of medication in elderly patients. The Journal of clinical psychiatry. 2001;62(21):11-14.

34. Mebarki S, Trivalle C. Échelles d'évaluation de l'effet anticholinergique des médicaments. NPG Neurologie-Psychiatrie-Gériatrie. 2012;12(69):131-138.

35. De Germay S, Lapeyre-Mestre M, Montastruc J-L, Montastruc F. Poids atropinique et médicaments anticholinergiques: intérêt et application en pratique clinique chez la personne âgée. Thérapie [Internet]. 2018;

36. HAS. Comment améliorer la qualité et la sécurité des prescriptions de médicaments chez la personne âgée? [Internet]. [cité 12 nov 2020]. Disponible sur : https://www.hassante.fr/jcms/c_1771468/fr/comment-ameliorer-la-qualite-et-la-securite-desprescriptions-de-medicaments-chez-la-personne-agee 
37. Bigot A. Comment prioriser les activités de pharmacie clinique dans les unités de soins ? élaboration d'un outil d'aide à la décision basé sur une analyse global des risques [Internet] [Thèse de doctorat]. Faculté de pharmacie Toulouse 1ll - Paul Sabatier ; 2015 [cité 15 nov 2020]. Disponible sur : http://thesesante.ups-tlse.fr/961/

38. Bernheim C, Schmitt E, Dufay E. Iatrogénie médicamenteuse nosocomiale et gestion des risques d'erreur médicamenteuse: à propos de l'analyse des notifications du réseau REEM. Oncologie. 2005;7(2):104-119.

39. Winterstein AG, Johns TE, Rosenberg EI, Hatton RC, Gonzalez-Rothi R, Kanjanarat P. Nature and causes of clinically significant medication errors in a tertiary care hospital. American Journal of Health-System Pharmacy. 2004;61(18):1908-1916.

40. Bates DW, Cullen DJ, Laird N, Petersen LA, Small SD, Servi D, et al. Incidence of adverse drug events and potential adverse drug events: implications for prevention. Jama. 1995;274(1):29-34.

41. Franklin BD, Reynolds M, Shebl NA, Burnett S, Jacklin A. Prescribing errors in hospital inpatients: a three-centre study of their prevalence, types and causes. Postgraduate medical journal. 2011;87(1033):739-745.

42. Bobb A, Gleason K, Husch M, Feinglass J, Yarnold PR, Noskin GA. The epidemiology of prescribing errors: the potential impact of computerized prescriber order entry. Archives of internal medicine. 2004;164(7):785-792.

43. Dean B, Schachter M, Vincent C, Barber N. Prescribing errors in hospital inpatients: their incidence and clinical significance. Quality and Safety in Health Care. 2002;11(4):340-344.

44. Lewis PJ, Dornan T, Taylor D, Tully MP, Wass V, Ashcroft DM. Prevalence, incidence and nature of prescribing errors in hospital inpatients. Drug safety. 2009;32(5):379-389.

45. Bates DW, Boyle DL, Vander Vliet MB, Schneider J, Leape L. Relationship between medication errors and adverse drug events. Journal of general internal medicine. 1995;10(4):199-205.

46. Lafuente-Lafuente C, Baudry É, Paillaud E, Piette F. Pharmacologie clinique et vieillissement. La Presse Médicale. 2013;42(2):171-80.

47. Fortin M, Krolak-Salmon P, Bonnefoy M. Analyse descriptive et comparative des différents modèles de fragilité. In: Chassagne P, Rolland Y, Vellas B, éditeurs. La personne âgée fragile [Internet]. Paris: Springer; 2009 [cité 15 nov 2020]. p. 11-26. Disponible sur : https://doi.org/10.1007/978-2-287-93911-2_2

48. Bergman H, Béland F, Karunananthan S, Hummel S, Hogan D, Wolfson C. Développement d'un cadre de travail pour comprendre et étudier la fragilité. Gérontologie et société. 2004;27(109):15-29.

49. Fried LP, Tangen CM, Walston J, Newman AB, Hirsch C, Gottdiener J, et al. Frailty in older adults: evidence for a phenotype. J Gerontol A Biol Sci Med Sci. 2001;56(3):M146-156. 
50. Bonnefoy M, Celarier T. Définition et Concept de Fragilité [Internet]. [cité 19 janv 2021]. Disponible sur : https:/www.auvergne-rhone-alpes.ars.sante.fr/system/files/ 2019-04/1.\%20D\%C3\%A9finition\%20et\%20Concept\%20de\%20Fragilit\%C3\%A9.pdf

51. SFDRMG-HAS. Prévention des chutes accidentelles chez la personne âgée, Recommandations, 2005 [Internet]. [cité 21 nov 2020]. Disponible sur : https://www.has-sante.fr/upload/docs/application/pdf/Prevention_chutes_recos.pdf

52. HAS. Note méthodologique et de synthèse documentaire. Prendre en charge une personne polypathologique en soins primaires, 2015 [Internet]. [cité 22 nov 2020]. Disponible sur : https://www.has-sante.fr/upload/docs/application/pdf/2015-04/note _methodologique_polypathologie_de_la_personne_agee.pdf

53. HAS. Prendre en charge une personne âgée polypathologique en soins primaires [Internet]. [cité 22 nov 2020]. Disponible sur : https://www.has-sante.fr/jcms/c 2028194/fr/prendre-en-charge-une-personne-agee-polypathologique-en-soins-primaires

54. Monégat M, Sermet C. La polymédication : définitions, mesures et enjeux. Revue de la littérature et tests de mesure. IRDES. Questions d'économie de la santé n $204 ; 2014$

55. Polypathologie et médicaments, iatropathologie [Internet]. Université Médicale Virtuelle Francophone; 1989 [Internet]. [cité 22 nov 2020]. Disponible sur : http://campus.cerimes .fr/geriatrie/enseignement/geriatrie11/site/html/cours.pdf

56. Courtois M. Etude observationnelle de la polymédication chez le patient de 50 à 74 ans en médecine générale [Internet] [Thèse de doctorat]. Faculté de médecine de Limoges ; 2017 [cité 22 nov 2020]. Disponible sur : http://aurore.unilim.fr/ori-oai-search/notice/ view/unilim-ori-75850

57. Linjakumpu T, Hartikainen S, Klaukka T, Veijola J, Kivelä S-L, Isoaho R. Use of medications and polypharmacy are increasing among the elderly. Journal of clinical epidemiology. 2002;55(8):809-817.

58. Routledge PA, O'Mahony MS, Woodhouse KW. Adverse drug reactions in elderly patients. British journal of clinical pharmacology. 2004;57(2):121-126.

59. Hovstadius B, Hovstadius K, Astrand B, Petersson G. Increasing polypharmacy-an individual-based study of the Swedish population 2005-2008. BMC clinical pharmacology. 2010;10(16).

60. Le Cossec C. La polymédication au regard de différents indicateurs de sa mesure: impact sur la prévalence, les classes thérapeutiques concernées et les facteurs associés. Les rapports de l'IRDES n562;2015

61. Thériaque [Internet]. [cité 18 janv 2021]. Disponible sur : https://www.theriaque.org /apps/contenu/accueil.php

62. DDI-Predictor Academic version. Prédiction quantitative des interactions médicamenteuses [Internet]. [cité 18 janv 2021]. Disponible sur : https://www.ddipredictor.org/ 
63. Laroche M. Le risque iatrogène chez la personne âgée : à propos des médicaments potentiellement inappropriés [Internet] [Thèse de doctorat]. Faculté de médecine de Limoges ; 2007 [cité 15 nov 2020]. Disponible sur : http://www.theses.fr/2007 LIMO310C

64. Legrain S. Mieux prescrire chez le sujet âgé en diminuant I' « underuse », la iatrogénie et en améliorant l'observance. Bulletin de l'Académie Nationale de Médecine. 2007;191(2):259-70.

65. Beers MH, Ouslander JG, Rollingher I, Reuben DB, Brooks J, Beck JC. Explicit criteria for determining inappropriate medication use in nursing home residents. UCLA Division of Geriatric Medicine. Arch Intern Med. 1991;151(9):1825-32.

66. Brehon E. Médicaments potentiellement inappropriés chez la personne âgée : étude portant sur 244 patients hospitalisés en gériatrie aiguë. [Internet] [Thèse de doctorat]. Faculté de pharmacie Picardie Jules Verne ; 2016 [cité 21 nov 2020]. Disponible sur : https://dumas.ccsd.cnrs.fr/dumas-01485611/document

67. Daret M. Modifications des prescriptions à la suite d'une hospitalisation en gériatrie : étude à la sortie, à trois mois, et à six mois, sur 80 patients de Lannemezan [Internet] [Thèse de doctorat]. Faculté de médecine de Toulouse III - Paul Sabatier ; 2019 [cité 21 nov 2020]. Disponible sur : http://thesesante.ups-tlse.fr/2862/

68. Fick DM, Cooper JW, Wade WE, Waller JL, Maclean JR, Beers MH. Updating the Beers criteria for potentially inappropriate medication use in older adults: results of a US consensus panel of experts. Arch Intern Med. 2003;163(22):2716-24.

69. Zhan C, Sangl J, Bierman AS, Miller MR, Friedman B, Wickizer SW, et al. Potentially inappropriate medication use in the community-dwelling elderly: findings from the 1996 Medical Expenditure Panel Survey. JAMA. 2001;286(22):2823-9.

70. McLeod PJ, Huang AR, Tamblyn RM, Gayton DC. Defining inappropriate practices in prescribing for elderly people: a national consensus panel. CMAJ. 1997;156(3):385-91.

71. Beers MH. Explicit criteria for determining potentially inappropriate medication use by the elderly. An update. Arch Intern Med. 1997;157(14):1531-6.

72. Laroche M-L, Bouthier F, Merle L, Charmes J-P. Médicaments potentiellement inappropriés aux personnes âgées : intérêt d'une liste adaptée à la pratique médicale française. La Revue de Médecine Interne. 2009;30(7):592-601.

73. Allouetteau S, Laroche M, Merle L, Charmes J. Les médicaments potentiellement inappropriés en gériatrie. Actualités Pharmaceutiques. 2011;50(511):24-9.

74. Renom-Guiteras A, Meyer G, Thürmann PA. The EU (7)-PIM list: a list of potentially inappropriate medications for older people consented by experts from seven European countries. European journal of clinical pharmacology. 2015;71:861-875.

75. O. Dalleur, A. Mouton, S. Marien, B. Boland. Stopp/Start, version 2, Un outil à jour pour la qualité de la prescription médicamenteuse chez les patients âgés de 65 ans et plus. Louvain médical 2015;134(5). 
76. Lang P, Boland B, Dalleur O. Prescription médicamenteuse inappropriée : les nouveaux critères STOPP/START. Revue Médicale Suisse. 2015;11:2115-23.

77. Lam MP, Cheung BM. The use of STOPP/START criteria as a screening tool for assessing the appropriateness of medications in the elderly population. Expert Review of Clinical Pharmacology. 2012;5(2):187-97.

78. Barry PJ, Gallagher P, Ryan C, O'Mahony D. START (screening tool to alert doctors to the right treatment) - an evidence-based screening tool to detect prescribing omissions in elderly patients. Age and ageing. 2007;36(6):632-638.

79. Gallagher P, Ryan C, Byrne S, Kennedy J, O’Mahony D. STOPP (Screening Tool of Older Person's Prescriptions) and START (Screening Tool to Alert doctors to Right Treatment). Consensus validation. International journal of clinical pharmacology and therapeutics. 2008;46(2):72-83.

80. O’Mahony D, O'Sullivan D, Byrne S, O'Connor MN, Ryan C, Gallagher P. STOPP/START criteria for potentially inappropriate prescribing in older people: version 2. Age and ageing. 2015;44(2):213-218.

81. Lang P-O, Dramé M, Guignard B, Mahmoudi R, Payot I, Latour J, et al. Les critères STOPP/START. v2: adaptation en langue française. NPG Neurologie-PsychiatrieGériatrie. 2015;15(90):323-336.

82. Croisier B, Kondo-Oestreicher M, Vogt-Ferrier N. Fonction rénale et adaptation posologique des médicaments en gériatrie: Gérontologie. Médecine et hygiène. 2004;62(2505):2295-2306.

83. Site GPR. Nouvelles recommandations de l'EMA pour les études chez l'insuffisant rénal [Internet]. 2016 [cité 18 janv 2021]. Disponible sur : http://sitegpr.com/fr/nouvellesrecommandations-de-lema-pour-les-etudes-chez-linsuffisant-renal/

84. Site GPR, le bon usage clinique du médicament [Internet]. [cité 18 janv 2021]. Disponible sur : http://sitegpr.com/fr/

85. Réseau Français des Centres Régionaux de Pharmacovigilance. Risques liés à une mauvaise observance thérapeutique. [Internet]. 2019 [cité 22 nov 2020]. Disponible sur : https://www.rfcrpv.fr/risques-lies-a-mauvaise-observance-therapeutique/

86. Bonnet-Zamponi, D, Legrain S. Prescription médicamenteuse et iatrogénie chez le sujet âgé. EM-Consulte. 2013;8(3):1-9.

87. Faroudja JM. La prescription et la place du médicament dans la relation médecin-patientpharmacien. Banque de données en santé publique [Internet]. 2012 [cité 29 nov 2020]. Disponible sur : http://bdsp-ehesp.inist.fr/vibad/index.php?action=getRecordDetail \&idt $=458355$

88. Kleinclauss, Penformis C A. Observance en pratique médicale et courante. EM-Consulte. 2013;8(4):1-6. 
89. JORF. Ordonnance $\mathrm{n}^{\circ}$ 2016-1729 du 15 décembre 2016 relative aux pharmacies à usage intérieur, Article L. 1110-12 [Internet]. [cité 24 nov 2020]. Disponible sur : https://www.legifrance.gouv.fr/eli/ordonnance/2016/12/15/AFSH1625422R/jo/texte

90. Société Française de Pharmacie Clinique (SFPC). Lexique de la Pharmacie Clinique. [Internet]. [cité 23 nov 2020]. Disponible sur : https://sfpc.eu/wpcontent/uploads/2019/11/1-s2.0-S2211104218302005-mmc1.pdf

91. ARS, OMEDIT PACA-Corse [Internet]. [cité 18 janv 2021]. Disponible sur : https://www.omeditpacacorse.fr/

92. JORF. Décret $\mathrm{n}^{\circ}$ 2011-375 du 5 avril 2011 relatif aux missions des pharmaciens d'officine correspondants [Internet]. [cité 30 nov 2020]. Disponible sur : https://www.legifrance.gouv.fr/eli/decret/2011/4/5/ETSH1105776D/jo/texte

93. JORF. Arrêté du 28 novembre 2016 relatif aux bonnes pratiques de dispensation des médicaments dans les pharmacies d'officine, les pharmacies mutualistes et les pharmacies de secours minières, mentionnées à l'article L. 5121-5 du code de la santé publique - Légifrance [Internet]. [cité 30 nov 2020]. Disponible sur : https://www.legifrance.gouv.fr/jorf/id/JORFTEXT000033507633/

94. Allenet B. Pour que l'éducation thérapeutique du patient devienne pour le pharmacien une posture de travail. Annales Pharmaceutiques Françaises. 2012;70(1):53-7.

95. Bourdillon F, Collin J-F. Une politique pour l'éducation thérapeutique du patient. La Presse Médicale. 2009;(38):1770-3.

96. Bouvenot G. La prescription des médicaments chez la personne âgée - Académie nationale de médecine. Une institution dans son temps. Académie nationale de médecine, Communiqué de séance [Internet]. 2012 [cité 22 nov 2020]. Disponible sur : http://www.academie-medecine.fr/la-prescription-des-medicaments-chez-la-personneagee-2/

97. Mezghani O, Orhan $\mathrm{P}$, Albertini L, Fauvelle F. Conciliation médicamenteuse à l'entrée du patient à l'unité d'hospitalisation de courte durée (UHCD) au GHI Le RaincyMontfermeil. Le Pharmacien Hospitalier et Clinicien. 2016;51(4):341.

98. ARS. Indicateurs du CAQES. Nationaux et régionaux. OMEDIT [Internet]. [cité 24 nov 2020]. Disponible sur : https://docplayer.fr/74923070-Indicateurs-du-caqes-nationauxet-regionaux-omedit.html

99. Quan H, Li B, Couris CM, Fushimi K, Graham P, Hider P, et al. Updating and Validating the Charlson Comorbidity Index and Score for Risk Adjustment in Hospital Discharge Abstracts Using Data From 6 Countries. American Journal of Epidemiology. 2011;173(6):676-82.

100. Bibmath. Statistique descriptive, inférentielle [Internet]. [cité 23 janv 2021]. Disponible sur : http://www.bibmath.net/dico/index.php?action=affiche\&quoi=./s/statistique.html

101. R-atique. Test de Wilcoxon-Mann-Whitney [Internet]. 2014 [cité 20 janv 2021]. Disponible sur : http://perso.ens-lyon.fr/lise.vaudor/test-de-wilcoxon-mann-whitney/ 
102. STHDA. Test de student - Formules [Internet]. [cité 20 janv 2021]. Disponible sur : http://www.sthda.com/french/wiki/test-de-student-formules

103. STHDA. Test exact de Fisher avec R [Internet]. [cité 21 janv 2021]. Disponible sur : http://www.sthda.com/french/wiki/test-exact-de-fisher-avec-r

104. XLSTAT. Modèle Logit conditionnel [Internet]. [cité 21 janv 2021]. Disponible sur : https://www.xlstat.com/fr/solutions/fonctionnalites/modele-logit-conditionnel

105. Minitab. Tests d'adéquation de l'ajustement pour la fonction Ajuster le modèle logistique binaire [Internet]. [cité 21 janv 2021]. Disponible sur : https://support.minitab.com/fr$\mathrm{fr} / \mathrm{minitab} / 18 /$ help-and-how-to/modeling-statistics/regression/how-to/fit-binarylogistic-model/interpret-the-results/all-statistics-and-graphs/goodness-of-fittests/\#hosmer-lemeshow

106. Boissinot L, Bachalat N, Perrier-Cornet E, Leglise P, Huchon-Bécel D. Conciliation des traitements médicamenteux en gériatrie: Pertinence et faisabilité. Pharmactuel. 2014;47(2):105-13.

107. Pérennes M, Carde A, Nicolas X, Dolz M, Bihannic R, Grimont P, et al. Conciliation médicamenteuse : une expérience innovante dans un service de médecine interne pour diminuer les erreurs d'anamnèse médicamenteuses. La Presse Médicale. 2012;41(3, Part 1):e77-86.

108. Bernard V, Rebai C, Ledoyen A, Roussel-Galle MC, Xavier-Ribot M, Beauclair P. Intégration de la conciliation médicamenteuse à une pratique pharmaceutique de terrain. Présentée à la session Hopipharm 2013. Congrès national des pharmaciens des hôpitaux. Lyon, 2013 [Internet]. [cité 22 nov 2020]. Disponible sur : https://www.synprefh. org/espace-congres/hopipharm/archives-hopipharm/communications-orales-afficheeslyon-2013

109. Cornish PL, Knowles SR, Marchesano R, Tam V, Shadowitz S, Juurlink DN, et al. Unintended Medication Discrepancies at the Time of Hospital Admission. Arch Intern Med. 2005;165(4):424-9.

110. Garriguet P, Beauverie P, Yassine S, Mouffak S, Alemanni J, Gaudoneix-Taïeb M, et al. Conciliation médicamenteuse en psychiatrie générale adulte: résultats de 3 années d'expérience. Le Pharmacien Hospitalier et Clinicien. 2017;52(2):138-46.

111. Boyer S. Contribution à l'étude du rôle de l'hyponatrémie légère et chronique dans la survenue de la chute grave du sujet âgé fragile admis en unité de Médecine d'Urgence de la Personne Agée (MUPA) [Internet] [Thèse de doctorat]. Université de Limoges ; 2018 [cité 15 nov 2020]. Disponible sur : https://www.theses.fr/2018LIMO0067

112. Henry C, Fellous L, Lagrange A, Chiarabini T, Bedos A, et al. Intérêt du dossier pharmaceutique dans la conciliation médicamenteuse à l'entrée du patient en service de médecine aiguë. Le Pharmacien Hospitalier et Clinicien. 2016;51(1):68.

113. Gutermann L, Marie A-S, Viguier F, Maire A, Zerhouni L, Benmelouka C, et al. Conciliation médicamenteuse d'entrée en milieu hospitalier: intérêt du dossier pharmaceutique. Le Pharmacien Hospitalier et Clinicien. 2016;51(1):67-8. 
114. Rédaction. Déploiement du Dossier Pharmaceutique: la généralisation est lancée! [Internet]. Hospitalia, le magazine de l'hôpital pour toute l'actualité et l'information hospitalière. [cité 24 janv 2021]. Disponible sur : https://www.hospitalia.fr/Deploiementdu-Dossier-Pharmaceutique-la-generalisation-est-lancee-_a1300.html

115. Moulis-Rivière M. Conciliation médicamenteuse de sortie en gériatrie : impact de la transmission hôpital-ville d'un bilan médicamenteux sur le maintien des optimisations thérapeutiques [Internet] [Thèse de doctorat]. Faculté de pharmacie de Grenoble ; 2014 [cité 25 janv 2021]. Disponible sur : https://dumas.ccsd.cnrs.fr/dumas-01021330/ document

116. Jean-Bart E, Faure R, Omrani S, Guilli T, Roubaud C, et al. Place du pharmacien clinicien dans l'optimisation thérapeutique en hôpital de jour gériatrique. Annales Pharmaceutiques Françaises. 2014;72(3):184-93.

117. Degeorges E. Analyse de prescriptions médicamenteuses dans un hôpital de jour gériatrique [Internet] [Thèse de doctorat]. Faculté de pharmacie de Lorraine ; 2017 [cité 25 janv 2021]. Disponible sur : https://hal.univ-lorraine.fr/hal-01932200/document 


\section{SERMENT DE GALIEN}

Je jure, en présence de mes maîtres de la Faculté, des conseillers de l'Ordre des pharmaciens et de mes condisciples:

* D'honorer ceux qui m'ont instruit dans les préceptes de mon art et de leur témoigner ma reconnaissance en restant fidèle à leur enseignement.

* D'exercer, dans l'intérêt de la santé publique, ma profession avec conscience et de respecter non seulement la législation en vigueur, mais aussi les règles de l'honneur, de la probité et du désintéressement.

* De ne jamais oublier ma responsabilité et mes devoirs envers le malade et sa dignité humaine, de respecter le secret professionnel.

* En aucun cas, je ne consentirai à utiliser mes connaissances et mon état pour corrompre les mours et favoriser des actes criminels.

Que les hommes m'accordent leur estime si je suis fidèle à mes promesses.

Que je sois couvert d'opprobre, méprisé de mes confrères, si j'y manque. 




\section{ETUDE OBSERVATIONNELLE DE FACTEURS PREDICTIFS D'ERREURS MEDICAMENTEUSES ET DE CRITERES DE PRIORISATION EN CONCILIATION MEDICAMENTEUSE GERIATRIQUE}

\section{Résumé du Mémoire :}

La population gériatrique, atteinte de différentes pathologies et polymédicamentée, est exposée aux risques d'évènements indésirables médicamenteux. La conciliation des traitements médicamenteux (CTM), processus interactif et pluriprofessionnel, garantit la continuité des soins. L'objectif de cette étude était d'analyser et de définir des critères de priorisation pertinents pour la CTM en gériatrie afin d'éviter le maximum d'erreurs médicamenteuses. Un audit clinique de la CTM réalisée aux points de transition que sont l'admission et la sortie du patient a été réalisé de façon prospective pendant 10 mois. Les patients ont été sélectionnés selon la procédure de priorisation déjà établie dans notre structure qui était la présence d'au moins un des trois critères suivants : provenance d'un service hospitalier, insuffisance rénale sévère et prescription de médicaments à risque. La cohorte de patients conciliés à l'entrée comprenait 136 patients. Les divergences intentionnelles étaient de 8 en moyenne par prescription. Au total, 63 divergences non intentionnelles (DNI) ont été recensées dont la majeure partie (76,2\%) impliquait des omissions de médicament. Trois critères ont été retenus comme facteurs prédictifs indépendants du risque de DNI sur l'ordonnance d'entrée par rapport au bilan médicamenteux optimisé : antécédents rhumatologiques, provenance d'un service hospitalier et hyponatrémie. L'hyponatrémie s'est révélée dans notre étude être le critère qui représentait significativement le plus de risque de présenter une DNI sur l'ordonnance du patient, notamment à risque d'omission de traitement à l'entrée. Cette étude permettra d'améliorer les critères de priorisation de la procédure de l'établissement de santé ainsi que de mettre en place la CTM en hospitalisation de jour gériatrique afin de renforcer le lien ville-hôpital. 